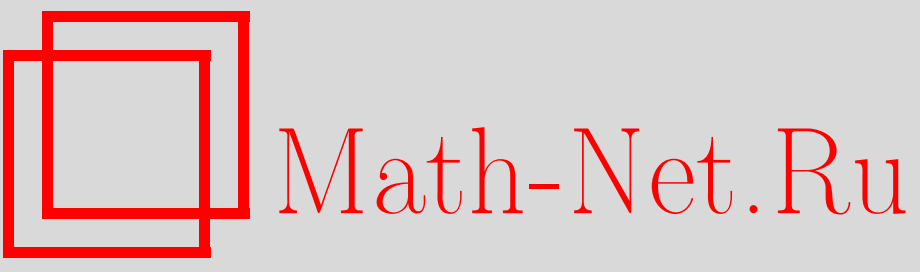

А. А. Махнев, Частичные геометрии и их расширения, УМH, 1999, том 54, выпуск $5,25-76$

DOI: https://doi.org/10.4213/rm203

Использование Общероссийского математического портала Math-Net.Ru подразумевает, что вы прочитали и согласны с пользовательским соглашением

http://www.mathnet.ru/rus/agreement

Параметры загрузки:

IP : 54.89 .56 .158

26 апреля 2023 г., 13:09:56 


\title{
ЧАСТИЧНЫЕ ГЕОМЕТРИИ И ИХ РАСШИРЕНИЯ
}

\author{
A. A. MAXHEB
}

Данный обзор состоит из трех частей: частичные геометрии, диаграммные геометрии и расширения частичных геометрий. В первой части помещены основные определения, все известные на данный момент примеры частичных геометрий, некоторые характеризационные теоремы, а также некоторые результаты о получастичных геометриях. Во второй части обзора приведены определения, относящиеся к диаграммным геометриям, ряд общих результатов об этих геометриях, примеры геометрий ранга 2, выступающих в роли строительных блоков для геометрий большего ранга, а также теоремы о строении некоторых флаг-транзитивных геометрий ранга 2. В третьей части содержится обзор результатов, характеризующих расширения частичных геометрий: 2-схем и дуальных 2-схем; сетей и дуальных сетей; а также обобщенных четырехугольников. Кроме того, в заключительном пункте этой части приведены некоторые обобщения понятия расширения и соответствующие характеризационные результаты.

Библиограбоия: 129 названий.

\section{СОДЕРЖАНИЕ}

Введение

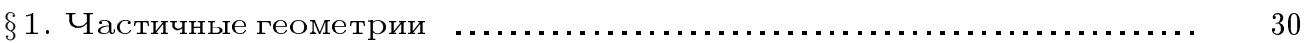

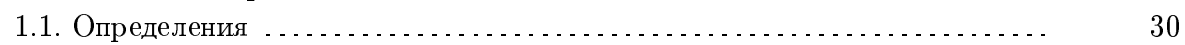

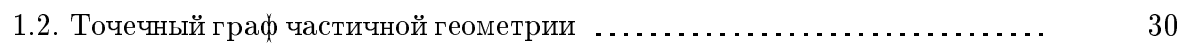

1.3. Известные примеры истинных частичных геометрий .............. 32

1.4. Некоторые характеризационные теоремы _..................... 36

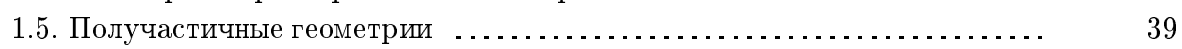

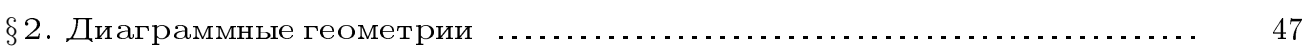

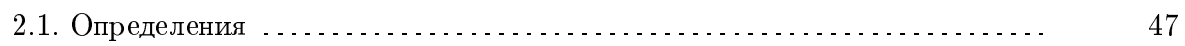

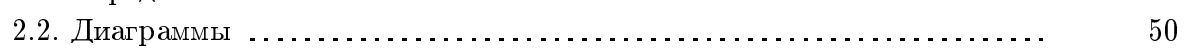

2.3. Некоторые диаграммные геометрии ранга 2 ......................... 51

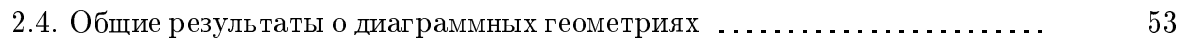

2.5. Флаг-транзитивные диаграммные геометрии ранга 2 .............. 53

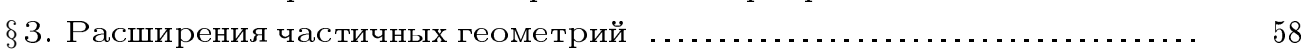

3.1. Расширения сетей и двойственных сетей ....................... 60

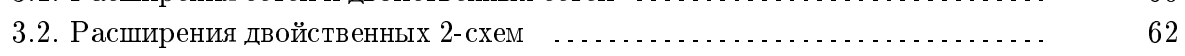

3.3. Расширения обобщенных четырехугольников .................... 66

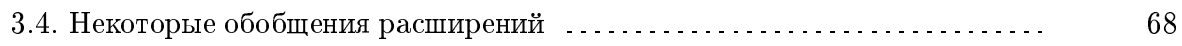

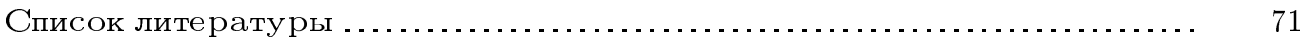




\section{Введение}

Данная статья является обзором достижений (в том числе и самых недавних) по частичным геометриям и их расширениям. Помешены также определения и некоторые обшие результаты по диаграммным геометриям. Публикаций на русском языке по последней теме до сих пор не было (кроме небольшого раздела в обзоре Кондратьева, Махнева, Старостина [73]). Среди обзоров на английском языке можно отметить работы Де Клерка, Ван Мальдегема [34] и Бюкенхаута, Пазини [15].

Система инцидентности $\mathbf{S}=(P, B, I)$ с множеством точек $P$, множеством блоков $B$ и симметричным отношением инцидентности $I \subseteq(P \times B) \cup(B \times P)$ назьвается геометрией ранга 2. Двойственной $\kappa \mathbf{S}$ назьвается следующая геометрия ранга 2 : $\mathbf{S}^{D}=\left(P^{D}, B^{D}, I^{D}\right)$, где $P^{D}=B, B^{D}=P$ и $I^{D}=I$. Если предположить, что в $B$ нет двух блоков, инцидентных одному и тому же множеству точек (нет кратных блоков), то каждьй блок можно отождествить с множеством инцидентных ему точек, отношение инцидентности становится симметризованным включением и геометрия обозначается через $\mathbf{S}=(P, B)$.

Для непустого подмножества $Q$ из $P$ имеется геометрия, назьваемая ограничениeм $\mathbf{S}$ на $Q$, с множеством точек $Q$ и множеством блоков $\{L \cap Q \mid L \in B\}$. Две точки из $P$ называются коллинеарными, если они лежат в общем блоке (заметим, что точка коллинеарна себе). Коллинеарность точек $x$ и $y$ (двойственно, факт пересечения блоков $L$ и $M$ ) мы будем обозначать как $x \sim y$ (соответственно $L \sim M$ ). Для точки $x$ положим $x^{\perp}=\{y \in P \mid y \sim x\}$. Вичетом геометрии $\mathbf{S}$ в точке $x$ называется геометрия $\mathbf{S}_{x}=\left(P_{x}, B_{x}, I_{x}\right)$, где $P_{x}=x^{\perp}-\{x\}, B_{x}=\{L \mid x \in L, L \in B\}$, $I_{x}=I \cap\left(\left(P_{x} \times B_{x}\right) \cup\left(B_{x} \times P_{x}\right)\right)$. Пара $(x, L) \in(P, B)$ называется флагом (антифлагом), если $x \in L(x \notin L)$.

Пример 0.1. Пусть $G$ - группа подстановок на множестве $P, H$ - подгруппа из $G$ и $L$ - орбита $H$ на $P$. Если рассмотреть множество блоков $B=\{L g \mid g \in G\}$, то геометрия $\mathbf{S}=(P, B)$ имеет флаг-транзитивную группу автоморфизмов $G$.

Для антифлага $(x, L)$ геометрии $\mathbf{S}$ через $\alpha(x, L)$ обозначим число точек, принадлежаших $L$ и коллинеарных $x$, эквивалентно, число блоков, содержаших $x$ и пересекающих $L$. Геометрия называется $\varphi$-однородной, если $\alpha(x, L)=0$ или $\varphi$ для любого антифлага $(x, L)$. Геометрия называется сильно $\varphi$-однородной, если $\alpha(x, L)=\varphi$ для любого антифлага $(x, L)$.

Если $R$ - отношение эквивалентности на подмножестве $Q$ из $P$ и $f$-каноническое отображение $Q$ на $Q / R$, то можно определить частное $(P, B) / R$ как геометрию с множеством точек $Q / R$ и множеством блоков $\{f(L \cap Q) \mid L \in B, P-Q \subseteq L\}$.

Пример 0.2. Пусть $G$ - группа подстановок на множестве $P$ и $a R b$ тогда и только тогда, ког да $a, b$ принадлежат общей орбите $G$ на $P$. Тогда $G$ действует тождественно на частном $(P, B) / R$.

Типичная иллюстрация для этой ситуации - это действие на правильном многограннике групшы, порожденной центральной симметрией (переставляющей антиподальные вершины).

Работа выполнена при финансовой поддержке Российского фонда фундаментальных исследований (грант № 99-01-00462) и при поддержке программой сотрудничества между Российской Академией Наук и Венгерской Академией Наук 1996-98 гг. 
ПримеР 0.3. Пусть $p$ - точка геометрии $(P, B)$. Определим частное $(P, B) / p$ с помошью отношения эквивалентности $R$ на $P-\{p\}$, положив $a R b$ тогда и только тогда, когда каждый блок, содержаший $a, p$, содержит $b$; и каждьй блок, содержаший $b, p$, содержит $a$. Здесь блоки частного $(P, B) / R$ определяются как $\{f(L-\{p\}) \mid$ $p \in L \in B\}$.

Если $R$ является отношением равенства на $P-\{p\}$, то частное $(P, B) / R$ совпадает с вычетом геометрии в точке $p$. Если $(P, B)$ - проективное или аффинное пространство, то частное в точке будет проективным пространством. Если же $(P, B)$ является $t$-схемой, то частное в точке будет обычной вычетной $(t-1)$-схемой.

Геометрия ранга 2 назьвается частичным пространством прямых, если каждьй ее элемент (элемент из $P \cup B$ ) инцидентен по крайней мере двум элементам и любые две точки инцидентны не более чем одному блоку, эквивалентно, любые два блока инцидентны не более чем одной точке. В этом случае говорят, что $B$ является множеством прямых. Прямую назовем тонкой, если она содержит точно две точки. Если все прямые частичного пространства прямых являются тонкими, то оно называется тонким. Если каждая точка лежит не менее чем на трех прямых и каждая прямая содержит не менее трех точек, то частичное пространство прямых называется толстым. Если любые две точки лежат на единственной прямой, то геометрия назьвается пространством прямых. Частичное пространство прямых назьвается $(0, \alpha)$-геометрией (Де Клерк и Тас [37]), если оно $\alpha$-однородно. Если каждая точка лежит на $t+1$ прямой, а каждая прямая содержит $s+1$ точек, $s, t \geqslant 1$, то $\mathbf{S}$ назьвается частичным пространством прямых порядка $(s, t)$. Легко проверить, что если $\mathbf{S}$ является $(0, \alpha)$-геометрией с $\alpha \geqslant 2$, то найдутся натуральные числа $s, t$ такие, что $\mathbf{S}$ имеет порядок $(s, t)$.

Геометрия $\mathbf{S}$ назьвается вложенной в проективное или аффинное пространство, если $B$ - это некоторое множество прямых этого пространства, а $P$ совпадает с множеством всех точек этого пространства, лежащих на прямых из $B$. Вложение геометрии порядка $(s, t)$ в $V=A G(n+1, s+1)$ назьвается линейным представлением, если $B$ является объединением параллельных классов прямых из $V$. В этом случае $P$ совпадает с множеством всех точек $V$, прямые из $B$ определяют в бесконечно удаленной гиперплоскости $\Pi_{\infty}$ множество $\mathscr{K}$ из $t+1$ точек. Мы обозначим соответствующее представление через $T_{n}^{*}(\mathscr{K})$. Прямую, пересекающую $\mathscr{K}$ по $m$ точкам, назовем $m$-секущей. Далее, 1-секущую назовем касательной, а 0-секущую - внешней прямой.

Граф с множеством вершин $P$ и отношением смежности, полученным удалением равенства из отношения коллинеарности, назьвается точечным графом геометрии $\mathbf{S}$. Скажем, что геометрия связна, регулярна и т. п., если этими свойствами обладает ее точечный граф. Аналогично определяется блочный граф геометрии ранга 2.

$\mathrm{C}$ другой стороны, граф иниидентности $\mathscr{I}(\mathbf{S})$ это граф с множеством вершин $P \cup B$, в котором две вершины смежны тогда и только тогда, когда соответствуюшие элементы инцидентны. Очевидно, геометрия определяется своим графом инцидентности. Далее, вершины графа одного типа могут быть соединены только путем четной длины. В частности, циклы из $\mathscr{I}(\mathbf{S})$ имеют четную длину. Если $2 g$ - обхват графа $\mathscr{I}(\mathbf{S})$, то параметр $g$ называется угольностью геометрии. Для вершины $x$ графа $Г$ локальным диаметром $d(x)$ называется наибольшая из длин геодезических путей в $Г$ 
с началом в $x$. Диаметром графа называется наибольший из локальных диаметров вершин. Через $d_{p}\left(d_{l}\right)$ обозначим максимум локальных диаметров $d(x)$ в графе $\mathscr{I}(\mathbf{S})$, когда $x$ является точкой (блоком) геометрии $\mathbf{S}$, а через $d$ - диаметр графа $\mathscr{I}(\mathbf{S})$ (т.е. максимум из двух чисел $\left.d_{p}, d_{l}\right)$. Наконец, граф флагов геометрии $\mathbf{S}$ имеет в качестве вершин флаги геометрии, и два различных флага смежны, если они имеют обшую вершину или общий блок. Через $d^{*}$ обозначим диаметр графа флагов.

Бюкенхаут [9] доказал, что связная геометрия $\mathbf{S}$, в которой каждый элемент инцидентен по крайней мере двум элементам и каждая точка (блок) имеет один и тот же локальньй диаметр $d_{p}\left(d_{l}\right)$, имеет диаметр $d^{*}$, равный минимуму из двух чисел $d_{p}, d_{l}$. В этом случае $\left\{d, d^{*}\right\}=\left\{d_{p}, d_{l}\right\}$, кроме того, всегда $g \leqslant d^{*}$ и $d-d^{*} \leqslant 1$. Такую геометрию Бюкенхаут назвал $\left(g, d_{p}, d_{l}\right)$-угольником. Иногда в это определение включают условие $d_{p} \leqslant g+2$, возникшее из наблюдения, что многие геометрии ранга 2 , построенные по конечным простым группам (особенно по спорадическим группам), удовлетворяют этому неравенству.

Реберным (линейным) графом данного графа Г называется граф, вершинами которого являются ребра $Г$, и две вершины смежны, если соответствующие ребра инцидентны общей вершине Г. Граф Г называется регулярным валентности $k$, если каждая его вершина смежна точно с $k$ вершинами. Дистаниионно регулярным графом с массивом пересечений $\left(b_{0}=k, b_{1}, \ldots, b_{d-1} ; c_{1}=1, c_{2}, \ldots, c_{d}\right)$ назьвается регулярньй связньй граф̆ диаметра $d$ такой, что для любой пары вершин $(x, y)$, находяшихся на расстоянии $j$, верны равенства

$$
\left|\Gamma_{j-1}(y) \cap \Gamma_{1}(x)\right|=c_{j} \quad(1 \leqslant j \leqslant d), \quad\left|\Gamma_{j+1}(y) \cap \Gamma_{1}(x)\right|=b_{j} \quad(0 \leqslant j \leqslant d-1) .
$$

Для любого графа Г диаметра $d$ с множеством вершин $\left\{x_{1}, \ldots, x_{v}\right\}$ матрицы расстояний $A_{h}, h=0, \ldots, d$, определяются как $(v \times v)$-матрицы с элементами

$$
\left(A_{h}\right)_{i j}= \begin{cases}1, & \text { если } d\left(x_{i}, x_{j}\right)=h, \\ 0 & \text { в противном случае. }\end{cases}
$$

Для дистанщионно регулярного граффа, имеющего массив пересечений $\left(b_{0}=k, b_{1}, \ldots\right.$, $\left.b_{d-1} ; c_{1}=1, c_{2}, \ldots, c_{d}\right)$, и $a_{i}=k-b_{i}-c_{i}$ верны равенства

$$
A_{1} A_{i}=b_{i-1} A_{i-1}+a_{i} A_{i}+c_{i+1} A_{i+1} \quad(1 \leqslant i \leqslant d-1),
$$

и $\left\{A_{0}=I, A_{1}, \ldots, A_{d}\right\}$ образуют базис соответствующей матричной алгебры (см. Броувер, Коэн и Ноймайер [4]).

Заметим, что любое ребро дистанционно регулярного графа лежит в $\lambda$ треугольниках, где $\lambda=k-b_{1}-1$, а каждая пара вершин, находяшихся на расстоянии 2 , имеет $\mu=c_{2}$ общих соседей. Дистанционно регулярный граф диаметра 2 называется сильно регулярным с параметрами $(v, k, \lambda, \mu)$, где $v$ - число вершин графа. Матрица смежности $A=A_{1}$ сильно регулярного графа удовлетворяет равенствам

$$
A J=k J, \quad A^{2}+(\mu-\lambda) A+(\mu-k) I=\mu J,
$$

где $J$ - матрица, все элементы которой равны 1. Таким образом, $A$ имеет три различных собственных значения $k, r, l$, причем $r+l=\lambda-\mu, r l=\mu-k$. В следующей теореме собраны различные необходимые условия сушествования сильно регулярного граб̆а (см. Броувер и ван Линт [5]). 
ТЕОРема 0.1. Если Г является сильно регулярным графом с параметрами $(v, k, \lambda, \mu)$, mo

(1) $k(k-\lambda-1)=\mu(v-k-1)$ (прямоугольное соотношение);

(2) кратности собственных значений $r>0$ u $l<0$ равны

$$
f=\frac{-k(l+1)(k-l)}{(k+r l)(r-l)} \quad u \quad g=\frac{k(r+1)(k-r)}{(k+r l)(r-l)} \quad \text { соответственно }
$$

(требование, что данные дроби должсны быть натуральными числами, называется условием иелочисленности);

(3) либо Г имеет параметры $(4 \mu+1,2 \mu, \mu-1, \mu)$, либо собственные значения $r$ u $l$ иелье

(4) дополнительный граф $\bar{\Gamma}$ также сильно регулярен $c$ параметрами $\bar{k}=v-k-1, \bar{\lambda}=v-2 k+\mu-2, \bar{\mu}=v-2 k+\lambda u$ собственными значениями $\bar{k}, \bar{r}=-(l+1), \bar{l}=-(r+1)$;

(5) выполняются условия Крейна:

$$
(r+1)(k+l+2 r l) \leqslant(k+r)(l+1)^{2}, \quad(l+1)(k+l+2 r l) \leqslant(k+l)(r+1)^{2} ;
$$

(6) верна абсолютная граница: $v \leqslant f(f+3) / 2, v \leqslant g(g+3) / 2$;

(7) верна граница для числа лап:

$$
\text { ecлu } \quad \mu \neq l^{2}, \quad \mu \neq l(l+1), \quad \text { mo } \quad 2(r+1) \leqslant l(l+1)(\mu+1) .
$$

Размеры клик и коклик в сильно регулярном графе ограничены сверху (см. Броувер и ван Линт [5]).

ТЕОРема 0.2. Пусть Г является сильно регулярнылм графом с параметрами $(v, k, \lambda, \mu)$ и собственными значениями $k, r, l$. Тогда

(1) $|L| \leqslant 1-k / l$ для любой клики $L$ из $\Gamma$, причем равенство достигается тогда и только тогда, когда каждая вериина вне $L$ смежна точно $c$ $\mu /(-l)$ вершинами из $L$ (граница Хоффмана для клик);

(2) $|C| \leqslant v(-l) /(k-l)$ для любой коклики $C$ из $\Gamma$, причем равенство достигается тогда и только тогда, когда кажсая вериина вне $C$ смежсна точно $c-l$ вериинами из $C$ (граница Хоффмана для коклик);

(3) размер любой коклики $C$ из Г не превосходит $g=\frac{k(r+1)(k-r)}{(k+r l)(r-l)}($ граница Цветковича для коклик), причем из равенств $|C|=g=v(-l) /(k-l)$ следует сильная регулярность графа, индуиированного $Г-C$.

Если граф̆ Г зафиксирован, то для вершины $a \in \Gamma$ через [a] обозначим окрестность $a$, через $a^{\perp}-$ подграф, индуцированный $\{a\} \cup[a]$, а через $[a]^{\prime}-$ антиокрестность $a$, т.е. подграфи, индуцированньй $\Gamma-a^{\perp}$. Для подмножества вершин $X$ графа Г положим

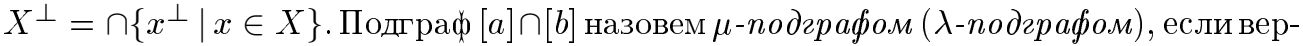
шины $a, b$ находятся на расстоянии 2 (смежны). Граф Г называется редуиированным, если для любых вершин $a, b$ из равенства $a^{\perp}=b^{\perp}$ следует $a=b$. 


\section{$\S$ 1. Частичные геометрии}

1.1. Определения. Сильно $\alpha$-однородное частичное пространство прямых порядка $(s, t)$ назьвается частичной геометрией и обозначается $p G_{\alpha}(s, t)$. Двойственная геометрия к $p G_{\alpha}(s, t)$ является частичной геометрией $p G_{\alpha}(t, s)$.

Частичные геометрии могут быть размешены в следующие четыре класса, имеюшие небольшие пересечения.

(а) Частичные геометрии с $\alpha=1$, т.е. обобщенные четырехугольники $G Q(s, t)$ (информацию о $G Q$ можно найти в монографии Пэйна и Таса [100]).

(б) Частичные геометрии с $\alpha=s+1$ или, двойственно, с $\alpha=t+1$, т.е. $2-(v, s+1,1)$ схемы или двойственные 2-схемы.

(в) Частичные геометрии с $\alpha=t$ или, двойственно, с $\alpha=s$, т.е. сети или двойственные сети. Положив для удобства $n=s+1, m=t+1$, мы скажем, что $p G_{t}(s, t)$ является сетью типа $(n, m)$.

(г) Истинные частичные геометрии с $1<\alpha<\min \{s, t\}$.

1.2. Точечный граф частичной геометрии. Точечный граф частичной геометрии $p G_{\alpha}(s, t)$ является сильно регулярным с параметрами $v=(s+1)(1+s t / \alpha)$, $k=s(t+1), \lambda=s-1+t(\alpha-1), \mu=\alpha(t+1)$. Отметим, что $p G_{\alpha}(s, t)$ имеет $b=(t+1)(1+s t / \alpha)$ прямых.

1.2.1. Псевдогеометрический граф. Сильно регулярный граф, имеющий указанные выше параметры, называется псевдогеометрическим для $p G_{\alpha}(s, t)$. В таких графах достигается граница Хоффмана для клик, и каждая вершина вне $(s+1)$-клики $L$ смежна с $\alpha$ вершинами из $L$. Если граф $Г$ является точечным графом некоторой частичной геометрии, то он называется геометрическим. Одной из важных проблем является проблема существования геометрического графа в классе псевдогеометрических графов с данными параметрами.

ТЕОРема 1.1 (Боуз [3]). Псевдогеометрический для $p G_{\alpha}(s, t)$ граф Г является геометрическим, если

$$
2(s+1)>t(t+1)+\alpha(t+1)\left(t^{2}+1\right)
$$

В действительности для графов, удовлетворяющих этому неравенству, необходимо $\alpha=t$ или $t+1$ (см. Броувер и ван Линт [5]).

Пусть граф̆ $Г$ является псевдогеометрическим для $p G_{\alpha}(s, t)$. Тогда $r=s-\alpha$, $l=-t-1$, из условий целочисленности следует, что $\alpha(s+t+1-\alpha)$ делит $s t(s+1)(t+1)$, условие Крейна принимает вид $(s+1-2 \alpha) t \leqslant(s-1)(s+1-\alpha)^{2}$.

1.2.2. Псевдогеометрические графы и условие Крейна. Если $\alpha=1$ (и $s \neq 1$ ), то условие Крейна преврашается в хорошо известное неравенство Хигмэна для обобщенных четырехугольников: $t \leqslant s^{2}$. В работе Камерона, Геталса и Зейделя [21] доказано, что псевдогеометрический граф для $G Q\left(s, s^{2}\right)$ является геометрическим. Там же сформулирована

ПроБЛЕмА 1. Будет ли геометрическим псевдогеометрический граф для $p G_{\alpha}(s, t)$ с $\alpha \geqslant 2$, для которого достигается равенство в условии Крейна? 
ПРЕДЛОЖЕНИЕ 1.1 (Махнев [84]). Пусть для псевдогеометрического графа геометрии $p G_{\alpha}(s, t), \alpha \geqslant 2$, достигается равенство в условии Крейна, $a \in \Gamma$. Тогда

(1) граф $\Lambda=[a]$ также является псевдогеометрическим для частичной геометрии $p G_{\alpha-1}(s-1, x)$, где $(\alpha-1) t=x(s-1)$;

(2) если $\alpha=2$, то $s=4,5,7$ и $t=27,32,54$ соответственно;

(3) если для графа $\Lambda=[a]$ также достигается равенство в условии Крейна, $m o s=2 \alpha$.

Граф Маклафлина является единственным псевдогеометрическим графом для $p G_{2}(4,27)$ (Геталс и Зейдель [52]), но вопрос о его геометричности остается открытьм.

Теорема 1.2 (Махнев [86]). Псевдогеометрический граф для $p G_{2}(5,32)$ (сильно регулярный граф с параметрами $(486,165,36,66))$ не существует.

В связи с теоремой 1.2 и предложением 1.1 представляют интерес следующие две проблемы.

ПроБлема 2. Существует ли псевдогеометрический граф для частичной геометрии $p G_{2}(7,54)$ ?

ПроБлемА 3. Существует ли частичная геометрия $p G_{2}(7,54)$ ?

Пусть для псевдогеометрического граффа частичной геометрии $p G_{\alpha}(s, t)$ и для окрестности его вершины достигается равенство в условии Крейна. Известно сушествование только одного графа с этим свойством (это снова граф Маклафлина). Если $\alpha=3$, то окрестность вершины в Г является псевдогеометрическим графом для $p G_{2}(5,32)$, и в силу теоремы 1.2 псевдогеометрический граф для $p G_{3}(6,80)$ не существует. Вопрос о сушествовании таких графов с $\alpha \geqslant 4$ остается открытым.

1.2.3. Некоторые классы псевдогеометрических графов. Точечный граф $\mathcal{S} p(2 m-1, q)$ симплектической полярности $P G(2 m-1, q)$, вершинами которого являются точки из $P G(2 m-1, q)$, и две точки смежны, если они лежат на вполне изотропной прямой, является псевдогеометрическим с $s=q\left(q^{m-1}-1\right) /(q-1), t=q^{m-1}$, $\alpha=\left(q^{m-1}-1\right) /(q-1)$.

Граф $N^{+}(2 m-1,2)$, вершинами которого являются точки из $P G(2 m-1,2)$, не принадлежашие квадрике $Q^{+}(2 m-1,2)$, и две точки смежны, если они лежат на прямой, касательной к квадрике, является псевдогеометрическим с $s=2^{m-1}-1, t=2^{m-1}$, $\alpha=2^{m-2}$.

Граф $N^{-}(2 m-1,2)$, вершинами которого являются точки из $P G(2 m-1,2)$, не принадлежашие квадрике $Q^{-}(2 m-1,2)$, и две точки смежны, если они лежат на прямой, касательной к квадрике, является псевдогеометрическим с $s=2^{m-1}+1, t=2^{m-2}$, $\alpha=2^{m-2}$.

Точечный граф $\Gamma Q(2 m, q)$ квадрики $Q(2 m, q)$ является псевдогеометрическим с $s=$ $q\left(q^{m-1}-1\right) /(q-1), t=q^{m-1}, \alpha=\left(q^{m-1}-1\right) /(q-1)$. Если $q$ четно, то граф $\Gamma Q(2 m, q)$ изоморфен $\mathcal{S} p(2 m-1, q)$.

Точечный граф $\Gamma Q^{+}(2 m-1, q)$ квадрики $Q^{+}(2 m-1, q)$ является псевдогеометрическим только при $m=2$ или 3 с $s=q\left(q^{m-1}-1\right) /(q-1), t=q^{m-2}$, $\alpha=\left(q^{m-1}-1\right) /(q-1)$. Если $m=2$, то мы получим точечный граф обобшенного 
четырехугольника $G Q(q, 1)$, а если $m=3$, то - точечньй граф двойственной схемы для точек и прямых $P G(3, q)$.

Точечный граф̆ $\Gamma Q^{-}(2 m-1, q)$ квадрики $Q^{-}(2 m-1, q)$ является псевдогеометрическим с $s=q\left(q^{m-2}-1\right) /(q-1), t=q^{m-1}, \alpha=\left(q^{m-2}-1\right) /(q-1)$. Если $m=3$, то мы получим точечный графф обобшенного четырехугольника $Q^{-}(5, q)$.

В следующей теореме собраны некоторые факты о геометричности перечисленных графов.

ТЕОРема 1.3. (1) Если $m=2 n>4$, то $N^{+}(2 m-1,2)$ является геометрическим (Де Клерк, Дай и Тас [30]).

(2) Граф $N^{-}(5,2)$ не является геометрическим (Де Клерк, Геверт и Тас [33]).

(3) Граф $\mathcal{S} p(3, q)$ является точечным графом обобщенного четырехугольника $W(q)$, графы $\mathcal{S} p(5, q), \mathcal{S} p(7, q)$ не являются геометрическими (Де Клерк, Геверт u Tac [33]).

\section{3. Известные примеры истинных частичных геометрий.}

1.3.1. Частичные геометрии $\mathbf{S}(\mathscr{K})$. Это бесконечное семейство было сконструировано Тасом [113] и независимо Уоллесом [126]. Подмножество $X$ из $n$ точек проективной плоскости называется $(n, d)$-дугой, если любая прямая пересекает $X$ не более чем по $d$ точкам. Максимальная $(n, d)$-дуга $\mathscr{K}$ проективной плоскости $\pi$ порядка $q$ имеет $n=q d-q+d, d \leqslant q$, причем любая секущая пересекает $\mathscr{K}$ по $d$ точкам. Для максимальной $(q d-q+d, d)$-дуги $\mathscr{K}$ множество прямых $\mathscr{K}^{\prime}$ плоскости $\pi$, не пересекаюших $\mathscr{K}$, будет максимальной $(q(q-d+1) / d, q / d)$-дугой двойственной плоскости.

Для максимальной $(q d-q+d, d)$ - дуги $\mathscr{K}$ определим систему иншидентности $\mathbf{S}(\mathscr{K})=$ $(P, B, I)$. Множество $P$ состоит из точек $\pi$, не лежащих в $\mathscr{K}, B$ состоит из прямых $\pi$, пересекающих $\mathscr{K}$. Инцидентность та же, что и в $\pi$. Тогда $\mathbf{S}(\mathscr{K})$ является частичной геометрией с $s=q-d, t=q-q / d, \alpha=q-q / d-d+1$.

В проективной плоскости $P G\left(2,2^{h}\right)$ для $0<m<h$ имеются $\left(2^{h+m}-2^{h}+2^{m}, 2^{m}\right)$ дуги, дающие частичные геометрии с $s=2^{h}-2^{m}, t=2^{h}-2^{h-m}, \alpha=\left(2^{m}-1\right) \times$ $\left(2^{h-m}-1\right)$. Геометрия $\mathbf{S}(\mathscr{K})$ является обобшенным четырехугольником, только если $h=2$, и это будет единственный обобшенный четырехугольник порядка 2.

Если $m=h-1, h \geqslant 2$, то точечньй граф̆ $\mathbf{S}(\mathscr{K})$ является котреугольньм графом $\overline{T\left(2^{h}+2\right)}$. Хотя эти графы однозначно определяются своими параметрами, это не влечет единственность геометрии. Например, Мэтон [92] доказал, что сушествует точно две геометрии $p G_{3}(4,6)$ (с точечным графом $\left.\overline{T(10)}\right)$. С другой стороны, котреугольньй граф $\overline{T(2 n)}$ является псевдогеометрическим для $p G_{n-2}(n-1,2 n-4)$, но графы $\overline{T(8)}$ (и дополнения графов Чанга, имеюшие те же параметры, что $\overline{T(8)}$ ) и $\overline{T(12)}$ не являются геометрическими (Де Клерк [28], Лэм и др. [74]).

1.3.2. Частичные геометрии $T_{2}^{*}(\mathscr{K})$. Пусть $\mathscr{K}$ является $(q d-q+d, d)$-дугой проективной плоскости $\pi$ порядка $q$, вложенной в $P G(3, q)$. Определим систему инцидентности $T_{2}^{*}(\mathscr{K})=(P, B, I)$. Множество $P$ состоит из точек $P G(3, q)$, не лежащих в $\pi, B$ состоит из всех прямых $P G(3, q)$, пересекаюших $\mathscr{K}$ по единственной точке, инцидентность та же, что и в $P G(3, q)$. Тогда $T_{2}^{*}(\mathscr{K})$ является частичной геометрией с $s=q-1, t=(q+1)(d-1), \alpha=d-1$. Это бесконечное семейство впервые было построено Тасом [113]. 
В проективной плоскости $P G\left(2,2^{h}\right)$ для $0<m<h$ имеются $\left(2^{h+m}-2^{h}+2^{m}, 2^{m}\right)$ дуги, дающие частичные геометрии с $s=2^{h}-1, t=\left(2^{h}+1\right)\left(2^{m}-1\right), \alpha=\left(2^{m}-1\right)$. Геометрия $T_{2}^{*}(\mathscr{K})$ является обобшенным четырехугольником, если $m=1$. Тогда $\mathscr{K}$ - гиперовал из $\pi$ и $s=2^{h}-1, t=2^{h}+1$.

1.3.3. Частичные геометрии $P Q^{+}(4 n-1, q), q=2$ или 3 . Пусть $Q^{+}=$ $Q^{+}(2 m-1, q)$ - гиперболическая квадрика в $P G(2 m-1, q)$. Множество максимальных вполне сингулярных подпространств разбивается на два класса $\mathscr{D}_{1}$ и $\mathscr{D}_{2}$. Два подпространства попадают в один класс, если коразмерность их пересечения имеет ту же четность, что и $m-1$.

Пусть $q$ нечетно, $x, y \in P G(2 m-1, q)-Q^{+}$. Точки $x, y$ назьваются эквивалентными, если существует такая несингулярная точка $z$, что прямые $x z$ и $y z$ являются касательными к $Q^{+}$. Это отношение можно определить следующим образом. Вложим $Q^{+}$в несингулярную гиперквадрику $Q$ из $P G(2 m, q)$ и полюс гиперплоскости $P G(2 m-1, q)$ относительно $Q$ обозначим через $p$. Точки $x, y$ эквивалентны, если прямые $x p$ и $у р$ являются либо обе секушими, либо обе внешними прямьпи к $Q$. То, что это отношение будет отношением эквивалентности, доказано Тасом [115]. Это отношение имеет два класса эквивалентности $E_{1}$ и $E_{2}$, причем для некоторого $i$ проекция $Q$ на $P G(2 m-1, q)$ относительно $p$ совпадает с $Q^{+} \cup E_{i}$.

Спредом $\Sigma$ гиперболической квадрики $Q^{+}=Q^{+}(4 n-1,2), n \geqslant 2$, называется семейство из $2^{2 n-1}+1$ попарно непересекаюшихся $(2 n-1)$-мерных подпространств из $Q^{+}$. Пусть $\Omega$ будет множеством всех гиперплоскостей элементов из $\Sigma$. Рассмотрим систему иншидентности $P Q^{+}(4 n-1,2)=(P, B, I)$. Множество $P$ состоит из точек $P G(4 n-1,2)$, не лежаших на квадрике, $B=\Omega$ и $x I L$ для $(x, L) \in(P, B)$ тог да и только тогда, когда $x \in L^{\perp}$, где $L^{\perp}$ - ортогональное к $L$ подпространство относительно $Q^{+}$. Тогда $P Q^{+}(4 n-1,2)$ является частичной геометрией с $s=2^{2 n-1}-1, t=2^{2 n-1}$, $\alpha=2^{2 n-2}$. Это бесконечное семейство впервые было построено Де Клерком, Даем и Тасом [30]. Заметим, что геометрия $P Q^{+}(7,2)$ является выгетом элемента типа 2 в геометрии ранга 3 спорадической группы Томпсона $F_{3}$ (см. Бюкенхаут [10]).

Неизоморфные спреды квадрики $Q^{+}(4 n-1,2)$ дают неизоморфные частичные геометрии. Если число $2 n-1$ составное, то $Q^{+}(4 n-1,2)$ имеет неизоморфные спреды (см. Кантор [70]).

Для $q=3$ аналогичная конструкция предложена Тасом [116]. Снова пусть $\Sigma$ является спредом гиперболической квадрики $Q^{+}=Q^{+}(4 n-1,3), \Omega$ будет множеством всех гиперплоскостей элементов из $\Sigma$. Рассмотрим систему иншидентности $P Q^{+}(4 n-1,3)=(P, B, I)$ с $P=E_{i}, B=\Omega$ и $x I L$ для $(x, L) \in(P, B)$ тогда и только тогда, когда $x \in L^{\perp}$. Тогда $P Q^{+}(4 n-1,3)$ является частичной геометрией с $s=3^{2 n-1}-1, t=3^{2 n-1}, \alpha=2 \cdot 3^{2 n-2}$.

Пока известно лишш, что $Q^{+}(7,3)$ имеет спред, это дает геометрию с $s=26, t=27$, $\alpha=18$.

ПроБЛЕма 4. Существуют ли частичные геометрии типа $P Q^{+}(4 n-1,3)$ для $n>2$ ?

1.3.4. Частичные геометрии $\mathscr{M}_{3}(n)$. Определим эрмитов граф̆ $\mathscr{H}(q)$ для нечетного $q$, являющегося степенью простого числа, в терминах эрмитовой параболы из 
$A G\left(2, q^{2}\right)$. Вершинами графа $\mathscr{H}(q)$ являются пары $(x, y)$, удовлетворяюшие уравнению $x^{q+1}+y+y^{q}=0$, вершины $\left(x_{1}, y_{1}\right),\left(x_{2}, y_{2}\right)$ смежны тогда и только тогда, когда $x_{1} x_{2}^{q}+y_{1}+y_{2}^{q}$ является квадратом или неквадратом в $G F\left(q^{2}\right)$ согласно $q \equiv 3$ или 1 по модулю 4. Известно (Броувер и ван Линт [5]), что $\mathscr{H}(q)$ является псевдогеометрическим с параметрами $s=q-1, t=\left(q^{2}-1\right) / 2, \alpha=(q-1) / 2$. Далее, граф̆ $\mathscr{H}(3)$ изоморфен точечному графу $G Q(2,4)$. Спенс $[110]$ доказал, что графы $\mathscr{H}(5)$ и $\mathscr{H}(7)$ не являются геометрическими.

Мэтон [93] построил новую бесконечную серию частичных геометрий $\mathscr{M}_{3}(n)$ с точечным графомо $\mathscr{H}\left(3^{2 n}\right)$.

Пусть $\beta$ - примитивньй элемент поля $G F\left(q^{2}\right), q=3^{2 n}$. Обозначим нулевой элемент поля через $\beta^{\infty}$ и положим $r=(q+1) / 2, q_{2}=q-2, q_{4}=\left(q^{2}-5\right) / 4, x_{k, l}^{i, \delta}=$ $\beta^{2 i+\delta}\left(\beta^{2 r k}-\beta^{2 r l-r}\right)$,

$$
y_{k, j, l}^{i, \delta}=\beta^{2 r(i+\delta)+r}\left[\beta^{2 r k}\left(\beta^{2 r k}+\beta^{2 r i-r}\right)^{2}-\beta^{2 r i-r}\left(\beta^{2 r k}-\beta^{2 r l-r}\right)^{2}\right]+\beta^{2 r j+r},
$$

где $k, l, j=\infty, 0,1, \ldots, q_{2}, i=0,1, \ldots, q_{4}$ и $\delta=0,1$.

Система инцидентности $\mathscr{M}_{3}(n)=(P, B, I)$ с множеством точек $P=\left\{\left(x_{k, l}^{0,0}, y_{k, j, l}^{0,0}\right)\right\}$ и множеством прямых $L=\left\{L_{j, l}^{i, \delta}, L_{k, l}^{\infty}\right\}$, где

$$
\begin{aligned}
& L_{j, l}^{i, \delta}=\left\{\left(x_{k, l}^{i, \delta}, y_{k, j, l}^{i, \delta}\right) \mid k=\infty, 0,1, \ldots, q_{2}\right\}, \\
& L_{k, l}^{\infty}=\left\{\left(x_{k, l}^{0,0}, y_{k, j, l}^{0,0}\right) \mid j=\infty, 0,1, \ldots, q_{2}\right\}
\end{aligned}
$$

$k, l, j=\infty, 0,1, \ldots, q_{2}, i=0,1, \ldots, q_{4}$ и $\delta=0,1$, является частичной геометрией с параметрами $s=3^{2 n}-1, t=\left(3^{4 n}-1\right) / 2, \alpha=\left(3^{2 n}-1\right) / 2$.

Группа автоморфизмов Aut $\mathscr{M}_{3}(n)$ является подгруппой индекса 4 в Aut $\mathscr{H}\left(3^{2 n}\right)$, действует транзитивно на множестве точек и имеет две орбиты на множестве прямых $S^{\infty}=\left\{L_{k, l}^{\infty}\right\}$, одну длины $3^{4 n}$ и вторую длины $3^{4 n}\left(3^{4 n}-1\right) / 2$.

1.3.5. Частичные геометрии $(V, \mathscr{L})$. Частичной $(\lambda, \mu)$-геометрией назовем систему инцидентности $(P, B)$, удовлетворяющую свойствам

$(S 1)$ каждый блок содержит $k$ точек из $P$, и каждая точка принадлежит $r$ блокам,

$(S 2)$ любые две точки принадлежат 0 или $\lambda$ блокам,

(S3) любые два блока пересекаются по 0 или $\mu$ точкам.

Если частичная $(\lambda, \mu)$-геометрия сильно $\beta$-однородна, то выполняется свойство

$(S 4)$ для любого антифлага $(a, B)$ имеется точно $\alpha=\beta \lambda / \mu$ блоков из $a^{\perp}$, пересекающих $B$.

Сильно $\beta$-однородная частичная $(\lambda, \mu)$-геометрия с параметрами $(k, r)$ содержит $v=k(r-1)(k-\mu) /(\beta \lambda)+k$ точек и $b=r(k-1)(r-\lambda) /(\beta \lambda)+r$ блоков, причем точечный граф и блочньй граф сильно регулярны с параметрами

$(v, r(k-1) / \lambda, r(\beta-1) / \lambda+k-\beta-1, r \beta / \lambda) \quad$ и $(b, k(r-1) / \mu, k(\alpha-1) / \mu+r-\alpha-1, k \alpha / \mu)$.

Для любого $m=2^{n}, n \geqslant 2$, существует сильно $(m+1)$-однородная частичная $(m, 2)$-геометрия $(P, B)$ с параметрами $v=m(m+2), k=m+2$ (Таюан Хуанг [65]). Пусть $G F(m)=\left\{f_{0}=0, f_{1}=1, f_{2}=\alpha, f_{3}=\alpha^{2}, \ldots, f_{m-1}=\alpha^{m-2}\right\}$, где $\alpha-$ 
примитивный элемент поля $G F(m)$. Положим $A_{h l}=\left(a_{i j}^{h l}\right)$, где $a_{i j}^{h l}=f_{i}+f_{j} f_{l}+f_{h} f_{l}^{2}$, $0 \leqslant h, i, j \leqslant m-1,1 \leqslant l \leqslant m-1$. Пусть $M_{h}$ означает ортогональный массив $O A(m+1, m)$, полученньй стандартным путем из семейства $\left\{A_{h 1}, \ldots, A_{h(m-1)}\right\}$, и $\bar{M}_{h}$ получается из $M_{h}$ добавлением новой первой строки $\left[\left(f_{h} f_{h} \ldots f_{h}\right)_{i}\right], 0 \leqslant i \leqslant m-1$. Таким образом, матрища $\bar{M}_{h}$ имеет вид

\begin{tabular}{|c|c|c|}
\hline$f_{h} f_{h} \ldots f_{h}$ & $f_{h} f_{h} \ldots f_{h}$ & $\ldots f_{h} \quad f_{h}$ \\
\hline$\overline{f_{0} f_{0} \ldots f_{0}}$ & $f_{1} f_{1} \ldots f_{1}$ & $\ldots f_{m-1} f_{m-1} \ldots f_{m-1}$ \\
\hline$f_{0} f_{1} \ldots f_{m-1}$ & $f_{0} f_{1} \ldots f_{m-1}$ & 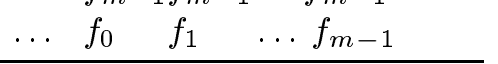 \\
\hline 0 -я строка $A_{h 1}$ & 1-я строка $A_{h 1}$ & $\ldots \quad(m-1)$-я строка $A_{h 1}$ \\
\hline 0-я строка $A_{h 2}$ & 1-я строка $A_{h 2}$ & $\ldots(m-1)$-я строка $A_{h 2}$ \\
\hline 0 -я строка $A_{h(m-1)}$ & 1-я строка $A_{h(m-1)}$ & $\ldots(m-1)$-я строка $A_{h(m-1)}$ \\
\hline
\end{tabular}

Для $0 \leqslant h \leqslant m-1$ если $i$-й столбец матрицы $\bar{M}_{h}$ имеет вид $\left[f_{b_{0}}\left(=f_{h}\right) f_{b_{1}} \ldots f_{b_{m+1}}\right]^{\mathrm{T}}$, $0 \leqslant b_{0}, b_{1}, \ldots, b_{m+1} \leqslant m-1$, то мы положим $B_{m^{2} h+i}=\left\{b_{l}+m l \mid 0 \leqslant l \leqslant m+1\right\}$. Если $P=\{0,1, \ldots, m(m+2)-1\}, B=\left\{B_{m^{2} h+i} \mid 0 \leqslant h \leqslant m-1,0 \leqslant i \leqslant m^{2}-1\right\}$, то $(P, B)$ - требуемая геометрия.

Пусть $V_{i}=\left\{\vec{x} \mid \vec{x}\right.$ пробегает множество столбцов матришы $\left.\bar{M}_{i}\right\}, 0 \leqslant i \leqslant m-1$, и $V=\bigcup V_{i}$. Для векторов $\vec{x} \in V_{0}, \vec{y} \in V_{1}$ положим $L(\vec{x}, \vec{y})=\left\{\vec{x}+f_{i}(\vec{x}+\vec{y}) \mid 0 \leqslant i \leqslant\right.$ $m-1\}$. Ясно, что $L(\vec{x}, \vec{y})$ состоит из $m$ векторов, включая $\vec{x}, \vec{y}$. Кроме того, положим

$$
\mathscr{L}=\left\{L(\vec{x}, \vec{y}) \mid \vec{x} \in V_{0}, \vec{y} \in V_{1}, d_{H}(\vec{x}, \vec{y})=m+2\right\}
$$

где $d_{H}$ - расстояние Хемминга.

Каждой сильно $(m+1)$-однородной частичной $(m, 2)$-геометрии $(P, B)$ с параметрами $v=m(m+2), k=m+2$ соответствует частичная геометрия $(V, \mathscr{L})$ c $s=m-1$, $t=m(m-1) / 2-1, \alpha=(m-2) / 2$ с точечным графом, изоморфным дополнению блочного граф̆а для $(P, B)$ (Хуанг [65]).

\subsection{6. Спорадическая частичная геометрия ван Линта-Шрайвера.}

Пусть $P=G F(81), \beta$ - примитивный элемент из $P$. Положим $\gamma=\beta^{16}, B=\{\{b, 1+b$, $\left.\left.\gamma+b, \gamma^{2}+b, \gamma^{3}+b, \gamma^{4}+b\right\} \mid b \in P\right\}$. Тогда $\mathbf{S}=(P, B, I)$ с симметризованным включением $I$ будет частичной геометрией с $s=t=5, \alpha=2$.

Известно, что точечный граф̆ этой геометрии является членом бесконечного семейства граффов, так назьваемого циклотомического типа (см. Калдебранк и Кантор [17]). Однако имеется предположение о том, что только один из этих графов является геометрическим.

1.3.7. Спорадическая частичная геометрия Хэмерса. Хэмерс [53] построил другую спорадическую частичную геометрию. Ее параметры равны $s=4, t=17$, $\alpha=2$. Хорошо известны сушествование и единственность сильно регулярного графа $\Lambda$ с параметрами $(50,7,0,1)$. Этот графф впервые был построен Хоффманом и Синглтоном [64]. Рассмотрим граф Г, вершинами которого являются ребра графа $\Lambda$, и два таких ребра смежны, если они не инцидентны обшей вершине и в $\Lambda$ соединены некоторым ребром. Тогда Г является псевдогеометрическим графом для $p G_{2}(4,17)$. Далее, 
Хэмерс нашел подмножество из 105 специальных графов Петерсена из $\Lambda$ такое, что каждый пятиугольник из $\Lambda$ содержится в единственном специальном графе Петерсена. Таким образом, система инцидентности из 175 вершин графа Г и 630 так называемых 1-факторов из специальных графов Петерсена образуют $p G_{2}(4,17)$.

Геометрия однозначно восстанавливается по вьшеуказанному точечному графуу. Более того, точечный граф̆ двойственной геометрии $p G_{2}(17,4)$ содержит точно 175 клик размера 18 , поэтому этот граф однозначно определяет $p G_{2}(4,17)$ (Сойчер, частное сообшение). Хэмерс [54] доказал, что эта частичная геометрия имеет одноточечное расширение до геометрии ранга 3 для группы Матье $M_{22}$.

\section{4. Некоторые характеризационные теоремы.}

1.4.1. Псевдогеометрические графы для $p G_{2}(4, t)$. Сильно регулярньй граф, имеюший параметры $(10 t+5,4 t+4, t+3,2 t+2)$, является псевдогеометрическим для $p G_{2}(4, t)$. Из условия целочисленности следует, что $t=1,2,3,7,9,12,17,27,57$. По условию Крейна $t \leqslant 27$. Для $t=1,2,17$ известно сушествование частичных геометрий, а для $t=27$ - сушествование и единственность графа (граф Маклафлина). Кроме того, для $t=3$ и 12 известно сушествование графа (частное графа Джонсона $\bar{J}(8,4)$ и эрмитов граф $\mathscr{H}(5))$. Для $t=7$ или 9 не известно сушествование ни указанных геометрий, ни сильно регулярных графов с данными параметрами. Автором [78] классифицированы псевдогеометрические графы для $p G_{2}(4, t)$ при условии, что все $\mu$-подграфы регулярны и не содержат треугольников. Оказалось, что последнее условие излишне.

ПреДЛОЖЕНИЕ 1.2 (Махнев [87]). Пусть Г - сильно регулярный граф с параметрами $(10 t+5,4 t+4, t+3,2 t+2)$. Тогда $\mu$-подграфы из Г не содержат треугольников.

Нецентрированной триадой назьвается такая 3-коклика, что пересечение окрестностей ее вершин пусто. Важность этого понятия объясняется тем, что если псевдогеометрический граф $\Gamma$ для частичной геометрии $p G_{2}(4, t)$ содержит максимальную 4 -клику $\Delta$, то $K_{0}(\Delta)$ является нецентрированной триадой.

Теорема 1.4 (Махнев [78], [87]). Пусть Г - псевдогеометрический граф для $p G_{2}(4, t)$. Тогда

(1) если Г имеет регулярные $\mu$-подграфь, то он является треугольныц графом T(6), частным графа Джонсона $\bar{J}(8,4)$ или графом Маклафлина;

(2) если Г содержит нецентрированную триаду, то он является треугольнымм графом Т(6) или частныцм графа Джсонсона $\bar{J}(8,4)$.

Как следствие получаем, чтов псевдогеометрическом графе для геометрии $p G_{2}(4, t)$ любой $\lambda$-подграф является объединением изолированных вершин и треугольников.

1.4.2. Графы для геометрий $\mathbf{c} \alpha=s-1$. Если $\mathbf{S}$ - частичная геометрия с $\alpha=s-1$, то дополнение точечного графа имеет собственное значение -2 . Сильно регулярные графы с собственным значением -2 были классифицированы Зейделем [106]. Класс Зейделевых графов включает полные многодольные графы $K_{n \times 2}$ с $n$ долями порядка 2 , решетчатые и треугольные графы, а также графы Шрикханде, Чанга, Петерсена, Клебша и Шлефли. 
Теорема 1.5 (Де Клерк [28]). Пусть Г - псевдогеометрический граф для $p G_{s-1}(s, t)$. Тогда выполняется одно из следующих утверждений.

(1) $s=2$ и $t=1,2$ или 4, Г является геометрическим и соответствующие обобщенные четырехугольники единственны.

(2) $s=3$ u $t=1,2$ илu 4 .

(a) если $t=1$, то Г является геометрическим и соответствующая частичная геометрия является единственной двойственной 2-схемой.

(b) если $t=2$, то Г является геометрическим и существуют точно две сети muna $(4,3)$.

(c) если $t=4$, то Г не является геометрическим.

(3) $s>3, t=s-1$ и Г является геометрическим тогда и только тогда, когда существует аффинная плоскость порядка $s+1$. Соответствующая сеть получается удалением двух параллельных классов из этой плоскости.

(4) $s>3, t=2 s-2$ и Г является геометрическим тогда и только тогда, когда проективная плоскость порядка $2 s$ содержит гиперовал О. Cоответствующая геометрия является двойственной $\kappa \mathbf{S}(\mathscr{O})$.

1.4.3. Частичные геометрии и аксиома Паша. Сначала сформулируем аксиому Паша $(\mathscr{P})$, иногда называемую аксиомой Веблена-Юнга.

Если $L_{1}, L_{2}$ - различные прямые, пересекающиеся в точке $x, x \notin M_{1} \cup M_{2}, u$ $L_{i} \sim M_{j}$ для всех $i, j \in\{1,2\}$, mo $M_{1} \sim M_{2}$.

Двойственная к ней аксиома часто называется диагональной аксиомой (D)). Любой обобщенньй четырехугольник тривиально удовлетворяет $(\mathscr{P})$ и $(\mathscr{D})$. Далее, $2-(v, s+1,1)$-схема удовлетворяет $(\mathscr{D})$. Любая 2 - $(v, s+1,1)$-схема, удовлетворяющая $(\mathscr{P})$, является проективным пространством. Единственная известная частичная геометрия с $\alpha \notin\{1, s+1, t+1\}$, удовлетворяюшая аксиоме Паша, - это двойственная сеть $H_{q}^{n+1}$. Она строится следующим образом. Пусть $H=P G(n-1, q)$ является подпространством из $\Sigma=P G(n+1, q)$. Тогда $H_{q}^{n+1}$ - это система инцидентности с множеством точек $\Sigma-H$, множеством прямых из $\Sigma$, не пересекающих $H$, и инцидентностью из $\Sigma$. Параметры этой двойственной сети $s=\alpha=q, t=q^{n}-1$.

Теорема 1.6 (Тас и Де Клерк [119]). Пусть $\mathbf{S}$ - двойственная сеть $p G_{s}(s, t)$ $(t+1>s)$, удовлетворяющая аксиоме Паша. Тогда $\mathbf{S}$ изоморфна $H_{q}^{n+1}$, в частности, $s=q u t+1=q^{n}$.

Заметим, что $H_{q}^{n+1}$ можно представить как дополнение сингулярной симплектической геометрии в $P G(n+1, q)$ с радикалом $H$. Далее, частичные пространства прямых, удовлетворяюшие $(\mathscr{P})$ и $(\mathscr{D})$, описаны Спрагу [111].

1.4.4. Вложения частичных геометрий в проективные и аффинные пространства. Имеется полная классификация частичных геометрий, вложимых в проективное пространство.

Теорема 1.7 (Де Клерк, Тас [36]). Пусть $\mathbf{S}=p G_{\alpha}(s, t)$ вкладивается в $P G(n, s)$, но не в $P G(m, s)$ для $m<n$. Тогда выполняется одно из утверәсдений.

(1) $\alpha=s+1$ и $\mathbf{S}$ является схемой точек и прямых $P G(n, s)$. 
(2) $\alpha=1$ и $\mathbf{S}$ - классический обобщенный четырехугольник (см. Бюкенхаут и Лефевр [13]).

(3) $\alpha=t+1, n=2$ и $\mathbf{S}$ является двойственной схемой $\kappa P G(2, s)$.

(4) $\alpha=s u \mathbf{S}=H_{q}^{n+1}$.

Имеется также полная классификация частичных геометрий, вложимых в аффинное пространство (Тас [114]). В случае обобшенных четырехугольников возникают интересные спорадические вложения (Пэйн и Тас [100]). Мы ограничимся формулировкой теоремы для истинных частичных геометрий.

ТЕОРема 1.8 (Тас [114]). Если $\mathbf{S}$ - истинная частичная геометрия, вложимая в аффинное пространство $A G(n, s+1)$, но не в $A G(m, s+1)$ для $m<n$, то $n=3$ и $\mathbf{S}=T_{2}^{*}(\mathscr{K})$, где $\mathscr{K}$ является максимальной дугой в бесконечно удаленной плоскости.

Для теорем, характеризующих $\mathbf{S}(\mathscr{K})$ и $T_{2}^{*}(\mathscr{K})$, мы отсылаем к Де Клерку, Де Сету и Геверту [31], Де Клерку, Геверту и Тасу [33] и Геверту [51].

1.4.5. Построение новых частичных геометрий с помощью заменяемых спредов. Следующие результаты содержатся в статье Де Клерка [29]. Пусть Фспред $p G_{\alpha}(s, t)$. Для прямой $L \notin \Phi$ через $\Phi_{L}$ обозначим множество из $s+1$ прямых в $\Phi$, пересекающих $L$. Прямую $L$ назовем регулярной относительно $\Phi$, если найдется множество из $s+1$ прямых $\mathscr{L}_{\Phi}=\left\{L_{0}=L, L_{1}, \ldots, L_{s}\right\}$, расщепляющее точечное множество $\mathscr{P}\left(\Phi_{L}\right)$, а любая прямая из $\mathscr{L}-\left(\mathscr{L}_{\Phi} \cup \Phi\right)$ пересекает $\mathscr{P}\left(\Phi_{L}\right)$ не более чем по $s$ точкам.

Если $L$ - регулярная прямая относительно спреда $\Phi$ частичной геометрии $p G_{\alpha}(s, t)$, то $t \geqslant s+1$. Если $t=s+1$, то каждая прямая из $\mathscr{L}-\left(\mathscr{L}_{\Phi} \cup \Phi\right)$ пересекает $\mathscr{P}\left(\Phi_{L}\right)$ по $\alpha$ точкам. Обратно, если каждая прямая из $\mathscr{L}-\left(\mathscr{L}_{\Phi} \cup \Phi\right)$ пересекает $\mathscr{P}\left(\Phi_{L}\right)$ по $\alpha$ точкам, то $t=s+1$.

Допустим, что $\Phi$ - такой спред, что каждая прямая регулярна относительно $\Phi$. Тогда $\mathscr{L}-\Phi$ расщепляется $s(s+1) / \alpha+1$ множествами $\mathscr{L}_{i}, i=0, \ldots, s(s+1) / \alpha$, причем каждое из них состоит из $s+1$ попарно не пересекающихся прямых. В этом случае спред $\Phi$ назовем заменяемым.

Если $\Phi$ - заменяемьй спред, то $D(\Phi)=\left(\Phi, \Phi_{L}\right)$ является $2-(s(s+1) / \alpha, s+1, \alpha)$ схемой.

Пусть $\mathcal{S}$ является частичной геометрией $p G_{\alpha}(s, t)$ с заменяемым спредом $\Phi$. Определим новую геометрию $\mathcal{S}_{\Phi}=\left(\mathscr{P}_{\Phi}, \mathscr{L}_{\Phi}\right)$ с $\mathscr{P}_{\Phi}=S \cup\left\{\mathscr{L}_{i} \mid i=0, \ldots, s(s+1) / \alpha\right\}$, $\mathscr{L}_{\Phi}=\mathscr{L}-\Phi$. Тогда $\mathcal{S}_{\Phi}$ является $p G_{\alpha}(s+1, s)$.

Мэтон и Стрит с помошью компьютера установили, что $P Q^{+}(7,2)$ содержит точно три заменяемые спреда, дающие (после дуализации) три неизоморфные частичные геометрии $p G_{4}(7,8)$. Одна из них снова содержит заменяемьй спред. В итоге Мэтон и Стрит с помошю этой техники (названной ими переключением (switching)) нашли семь новых частичных геометрий $p G_{4}(7,8)$, не изоморфных $P Q^{+}(7,2)$.

Де Клерк строит спреды типа $(i)$ в частичных геометриях $P Q^{+}(4 n-1, q)$ для $i=$ $1,2,3$ (последний тип лишь при четном $n$ ). Если $q=2$ или 3 , то спред типа (1) является заменяемьм, а спреды типов (2) и (3) заменяемы только при $n=2$. 
СлЕДСТВИЕ (Де Клерк [29]). Частичная геометрия $P Q^{+}(7, q), q=2,3$, имеет точно три спреда, каждый из которых заменяем.

\section{5. Получастичные геометрии.}

1.5.1. Определения. Получастичной геометрией $s p G_{\alpha}(s, t, \mu)$ называется $(0, \alpha)$-геометрия порядка $(s, t)$, в которой любые две неколлинеарные точки коллинеарны с $\mu$ общими точками.

Точечный граф $s p G_{\alpha}(s, t, \mu)$ является сильно регулярньм с параметрами $v=$ $1+(t+1) s(\mu+t(s-\alpha+1)) / \mu, k=s(t+1), \lambda=s-1+t(\alpha-1)$.

Получастичная геометрия с $\alpha=1$ называется частичным четырехугольником. Такие геометрии рассматривались Камероном [18]. Получастичные геометрии являются обобщением частичных геометрий и частичных четырехугольников. Заметим, что получастичная геометрия является частичной, если $\mu=\alpha(t+1)$. Получастичную геометрию с $\mu<\alpha(t+1)$ назовем истинной.

Двойственная к истинной получастичной геометрии снова будет получастичной геометрией, только если $s=t$ (Дебре и Тас [39]). В следующей теореме собраны условия на параметры получастичной геометрии.

Теорема 1.9 (Де Клерк и Ван Мальдегем [34]). Пусть $s p G_{\alpha}(s, t, \mu)$ является истинной получастичной геометрией. Тогда

(1) $t \geqslant s u b \geqslant v$;

(2) либо $D=(t(\alpha-1)+s-1-\mu)^{2}+4(s(t+1)-\mu)$ равно $5($ и $\mathbf{S}-$ пятиугольник $)$, либо $D$ является квадратом $и$

$$
\frac{2 s(t+1)+(v-1)(t(\alpha-1)+s-1-\mu+\sqrt{D})}{2 \sqrt{D}}
$$

- иелое число;

(3) $\alpha$ делит $\mu$ и $\alpha^{2} \leqslant \mu \leqslant \alpha(t+1)$;

(4) $\mu$ делит $(t+1) \operatorname{st}(s+1-\alpha)$;

(5) $\alpha$ делит $s t(t+1) u t s(s+1)$;

(6) $\alpha^{2}$ делum $\mu$ st u $t(\alpha(t+1)-\mu)$;

(7) $v(t+1) s$ четно;

(8) 3 делит $v(t+1) s(s-1) u v(t+1) s t(\alpha-1)$;

(9) 8 делит $v(t+1) s(s-1)(s-2) u v(t+1) s(t(\alpha-1)((t-1)(\alpha-1)-(\alpha-2))+$ $t(s+1-\alpha)(\mu-2 \alpha+1))$.

\subsection{2. Примеры истинных получастичных геометрий.}

Тонкие частичные четырехугольники. Сильно регулярный граф̆ $\Gamma$ с $\lambda=0$ является частичным четырехугольником с $s=1, t=k-1$, в частности, тонкой геометрией. Единственныеизвестныепримеры таких графов-этопятиугольник, граф Петерсена с параметрами $(10,3,0,1)$, граф Клебша с параметрами $(16,5,0,2)$, граф Хоффмана-Синглтона с параметрами $(50,7,0,1)$ и семейство графов Хигмена-Симса: граф Гевиртца с параметрами $(56,10,0,2)$, граф Хигмена-Симса с параметрами $(100,22,0,6)$ и антиокрестность вершины в этом графе с параметрами $(77,16,0,4)$. Любой из этих графов однозначно определяется своими параметрами. 
Получастичные геометрии $\overline{M(k)}, k \in\{2,3,7,57\}$. Графом Мура называется граф валентности $k$ обхвата 5 с числом вершин $v=k^{2}+1$. Такой граф $M(k)$ является сильно регулярным с $\lambda=0, \mu=1$ и $k \in\{2,3,7,57\}$. Примеры графов с $k=2,3,7$ приведены в предыдущем абзаце, все они являются графами ранга 3 . Существование $M(57)$ неизвестно (Ашбахер [1] доказал, что этот граф̆ не является графом ранга 3 , а Хигмен установил, что $M(57)$ не является вершинно транзитивным, см. Камерон [19]). Махнев и Падучих (Махнев [88]) выяснили строение группы автоморфизмов $G$ графа Ашбахера $M(57)$, при условии, что $G$ содержит инволюцию. Для подмножества $X$ из $G$ через $\operatorname{Fix}(X)$ обозначим подграф̆, индуцированный множеством вершин граф̆а, неподвижных относительно любого автоморфизма из $X$.

ПреДЛоЖенИЕ 1.3. Допустим, что группа автоморфизмов $G$ графа Ашбахера $M(57)$ содержит инволючию $t$. Тогда выполняются следующие утверждения:

(1) $\operatorname{Fix}(t)$ является звездой, содержащей 56 вершин;

(2) $G=Y\langle t\rangle \times X$ для некоторых подгрупп $X, Y$ нечетного порядка, $Y$ инвертируется $t$ и либо $|Y|$ делит 5 или 57, либо $|Y|$ делит 21 (в случае $|Y|=21$ имеем $|\operatorname{Fix}(y)|=37$ для әлемента у порядка 7 из $Y)$;

(3) если $X \neq 1$, то $\operatorname{Fix}(X)$ - либо звезда, $Y=1,|X|=7$; либо пятиугольник, $|Y|$ делит $5,|X|$ делит 55; либо граф Петерсена, $|Y|$ делит $3,|X|$ делит 27; либо граф Хоффмана-Синглтона, $|Y|$ делит 5 или $7,|X|$ делит 25.

С каждым графом Мура $\Gamma=M(k)$ соотносится другая получастичная геометрия $\overline{M(k)}$, точками которой являются вершины графа $\Gamma$, прямыми - окрестности вершин в $\Gamma$, инцидентность естественная. Эта геометрия имеет параметры $s=t=\alpha=k-1$, $\mu=(k-1)^{2}$ (Дебре и Тас [40]).

Геометрии $U_{2,3}(n)$. Пусть $U$ является $n$-элементным множеством, $P$ - множество неупорядоченных пар из $U, B$ - множество неупорядоченных троек из $U$, отношение инцидентности - включение. Тогда $U_{2,3}(n)=(P, B, I)$ является получастичной геометрией с параметрами $s=\alpha=2, t=n-3, \mu=4$ (Дебре и Тас [40]). Точечньй граф этой геометрии - треугольный граф $T(n)$.

Получастичные геометрии $L P(n, q)$. Пусть $P$ - множество прямых из $P G(n, q)(n \geqslant 4), B$ - множество плоскостей из $P G(n, q), I$ - отношение включения. Тогда $(P, B, I)$ является получастичной геометрией с параметрами $s=q(q+1)$, $t=\left(q^{n-1}-1\right) /(q-1)-1, \alpha=q+1, \mu=(q+1)^{2}$ (Дебре и Тас [40]). Заметим, что при $n=3$ получается двойственная схема прямых и плоскостей из $P G(3, q)$.

Геометрии $\overline{W(2 n+1, q)}$. Пусть $\sigma$ - симплектическая полярность $P G(2 n+1, q)$ $(n \geqslant 1), P$ - множество точек $P G(2 n+1, q)(n \geqslant 1), B$ - множество не вполне изотропных (гиперболических) прямых $P G(2 n+1, q), I$ - отношение включения. Тогда $(P, B, I)$ является получастичной геометрией с параметрами $s=q, t=q^{2 n}-1, \alpha=q$, $\mu=q^{2 n}(q-1)$ (Дебре и Тас [40]).

Получастичные геометрии $N Q^{ \pm}(2 n-1,2)$. Пусть $Q^{\varepsilon}$ является квадрикой в $P G(2 n-1,2), \varepsilon= \pm, P$ - множество точек квадрики, $B$ - множество прямых $P G(2 n-1,2)$, не содержашихся в $Q^{\varepsilon}, I$ - отношение включения. 
Тогда $N Q^{\varepsilon}(2 n-1,2)=(P, B, I)$ является получастичной геометрией с параметрами $s=\alpha=2, t=2^{2 n-3}-\varepsilon 2^{n-2}-1, \mu=2^{2 n-3}-\varepsilon 2^{n-1}$ (Вильбринк, не опубликовано).

Геометрии $H_{q}^{(n+1) *}$. Пусть $P$ - множество прямых из $\Sigma=P G(n+1, q)(n \geqslant 4)$, не пересекаюших данное проективное подпространство $H=P G(n-1, q), B$-множество плоскостей из $\Sigma$, пересекаюших $H$ по единственной точке, $I$ - отношение включения. Тогда $(P, B, I)$ является получастичной геометрией с параметрами $s=q^{2}-1, t=$ $\left(q^{n-1}-1\right) /(q-1)-1, \alpha=q, \mu=q(q+1)$.

Известно, что по любой получастичной геометрии $\mathbf{S}$, удовлетворяющей аксиоме $(\mathscr{D})$, можно построить новую получастичную геометрию $\overline{\mathbf{S}}$, также удовлетворяющую аксиоме $(\mathscr{D})$ (Де Клерк и Тас [36] и Дебре [38]). В самом деле, пусть $x, y$ - две коллинеарные точки, $D_{x, y}^{1}$ - множество всех коллинеарных с $x, y$ точек, не лежащих на прямой $x y, D_{x, y}^{2}$ - множество всех точек прямой $x y$, коллинеарных с точкой (и, значит, со всеми точками) $D_{x, y}^{1}$. Тогда $D_{x, y}=D_{x, y}^{1} \cup D_{x, y}^{2}$ является максимальным множеством попарно коллинеарных точек и назьвается диагональной кликой. Система инцидентности $\overline{\mathbf{S}}$ с тем же точечным множеством, что и $\mathbf{S}$, и с диагональными кликами в качестве прямых является получастичной геометрией с параметрами $\bar{s}=(t+1)(\alpha-1)$, $\bar{t}=s /(\alpha-1)-1, \bar{\alpha}=\alpha, \bar{\mu}=\mu$. Заметим, что $\overline{\mathbf{S}}$ имеет тот же точечньй граф, что и $\mathbf{S}$, и что $\overline{\overline{\mathbf{S}}}=\mathbf{S}$.

Указанным способом можно построить $H_{q}^{(n+1) *}$ из геометрии, двойственной к $H_{q}^{(n+1)}$.

1.5.3. Линейные представления получастичных геометрий. Если $T_{n}^{*}(\mathscr{K})$ - линейное представление получастичной геометрии, то $\mathscr{K}$ является таким множеством из $P G(n, q)$, что любая прямая будет внешней, касательной или $(\alpha+1)$-секушей, причем каждая точка из $P G(n, q)-\mathscr{K}$ лежит на $\mu /(\alpha(\alpha+1))$ секуших. Известны следуюшие примеры. Подмножество $X$ из $k$ точек проективного пространства $P G(n, q)$ назьвается $k$-чашкой, если любая прямая из $P G(n, q)$ пересекает $X$ не более чем по двум точкам.

Линейные представления частичных четырехугольников. В этом случае $\mathscr{K}$ является $(t+1)$-чашкой из $P G(n, q)$ со свойством, что каждая точка из $P G(n, q)-$ $\mathscr{K}$ лежит на $t+1-\mu$ касательных. Следующие утверждения дают классификацию линейных представлений истинных частичных четырехугольников. Утверждения 2-4 доказаны Калдебранком [16].

1. Частичньй четырехугольник $T_{3}^{*}(\mathscr{O})$, где $\mathscr{O}$ - овоид из $P G(3, q)$, имеет параметры $s=q-1, t=q^{2}, \mu=q(q-1)$ (впервые был построен Камероном [18]).

2. Если $q=2$, то $\mathscr{O}$ - эллиптическая квадрика из $P G(3,2)$, частичный четырехугольник $T_{3}^{*}(\mathscr{O})$ имеет параметры $s=1, t=4, \mu=2$.

3. Пусть $q=3$ и $\mathscr{K}-$ не овоид. Тогда $\mathscr{K}$ либо является 11 -чашкой из $P G(4,3)$ (см. Кокстер [25] или Пеллегрино [101]) и частичный четырехугольник $T_{4}^{*}(\mathscr{K})$ имеет параметры $s=2, t=10, \mu=2$, либо $\mathscr{K}$ - единственная 56-чашка из $P G(5,3)$ (см. Сегре [105]) и частичный четырехугольник $T_{5}^{*}(\mathscr{K})$ имеет параметры $s=2, t=55$, $\mu=20$.

4. Пусть $q=4$ и $\mathscr{K}$ - не овоид. Тогда $\mathscr{K}$ либо является 78 -чашкой из $P G(5,4)$ такой, что каждая внешняя точка лежит на 7 касательных (Хилл [60]), и частичньй 
четырехугольник $T_{5}^{*}(\mathscr{K})$ имеет параметры $s=3, t=77, \mu=14$, либо $\mathscr{K}-430$-чашка из $P G(6,4)$ (сушествование такой чашки неизвестно) и частичный четырехугольник $T_{6}^{*}(\mathscr{K})$ имеет параметры $s=3, t=429, \mu=110$.

5. Если $q \geqslant 5$, то Цанакис и Вольфскил [125] доказали, что частичный четырехугольник совпадает с $T_{3}^{*}(\mathscr{O})$, где $\mathscr{O}$ - овоид.

Сушествование такой чашки $\mathscr{K}$ в $P G(n, q)$, что любая внешняя точка лежит на одном и том же числе касательных, обеспечивает построение линейного $[|\mathscr{K}|, n+1]$ кода над $G F(q)$ с точно двумя ненулевыми весами (Калдебранк и Кантор [17]).

Линейные представления получастичных геометрий с $\alpha \geqslant 2$. Известны следующие примеры.

1. Множество $\mathscr{K}$ является униталью $\mathscr{U}$ проективной плоскости $\Pi_{\infty}=P G\left(2, q^{2}\right)$, и $T_{2}^{*}(\mathscr{U})$ имеет параметры $s=q^{2}-1, t=q^{3}, \alpha=q$ и $\mu=q^{2}\left(q^{2}-1\right)$.

2. Множество $\mathscr{K}$ является бэровским подпространством $\mathscr{B}$ проективного пространства $\Pi_{\infty}=P G\left(n, q^{2}\right)$, и $T_{n}^{*}(\mathscr{B})$ имеет параметры $s=q^{2}-1, t=\left(q^{n}-1\right) /(q-1)-1$, $\alpha=q$ и $\mu=q(q+1)$ (эта геометрия изоморфна $H_{q}^{(n+2) *}$ ).

\subsection{4. Получастичные геометрии и обобщенные четырехугольники.}

1. Для любой точки $р$ обобщенного четырехугольника $\mathbf{S}=(P, B, I)$ система инцидентности $\mathbf{S}^{p}=\left(P^{p}, B^{p}, I^{p}\right)$ с $P^{p}=P-p^{\perp}, B^{p}=B-B_{p}$ является $(0,1)$-геометрией порядка $(s-1, t)$, удовлетворяюшей следуюшему свойству.

(*) Если $L, M-$ две непересекающиеся прямые из $\mathbf{S}^{p}$, то имеется точно $0, s-1$ или $s$ прямых из $\mathbf{S}^{p}$, пересекающих $L$ и $M$.

Заметим, что это свойство тривиально вьполняется, если $s=2$. Точечньй граф $\Gamma\left(\mathbf{S}^{p}\right)$ будет сильно регулярным, если для любых двух неколлинеарных точек $x, y \in P^{p}$ подграф $\{p, x, y\}^{\perp}$ содержит точно $t+1-\mu$ вершин. Это условие вьполняется тогда и только тогда, когда $\mathbf{S}$ порядка $\left(s, s^{2}\right)$, причем в этом случае $\left|\{p, x, y\}^{\perp}\right|=s+1$ для любой тройки попарно неколлинеарных точек. Таким образом, частичньй четырехугольник $\mathbf{S}^{p}$ имеет параметры $\left(s-1, s^{2}, s(s-1)\right)$.

Иванов и Шпекторов [68] доказали, что сильно регулярный граф с параметрами $\left(q^{4},\left(q^{2}+1\right)(q-1), q-2, q(q-1)\right)$, в котором каждое ребро содержится в клике порядка $q$, является точечным графом частичного четырехугольника типа $\mathbf{S}^{p}$ и однозначно расширяется до обобшенного четырехугольника $\mathbf{S}$. Этот результат влечет, в частности, единственность сильно регулярного графа с параметрами $(81,20,1,6)$.

Напомним, что линейное представление частичного четырехугольника с параметрами $\left(s-1, s^{2}, s(s-1)\right)$ лежит в $A G(4, s)$, поэтому оно совпадает с $T_{3}^{*}(\mathscr{O})$, где $\mathscr{O}-$ овоид из $\Pi_{\infty}$.

Теорема 1.10 (Де Клерк и Ван Мальдегем [34]). Пусть $T_{n}^{*}(\mathscr{K})(n \geqslant 3)$ является линейным представлением $(0,1)$-геометрии порядка $(q-1, t), q>2$, удовлетворяющим (*). Если $\mathscr{K}$ порождает гиперплоскость $\Pi_{\infty}$, то $T_{n}^{*}(\mathscr{K})$ является частичныцм четырехугольником $T_{3}^{*}(\mathscr{O})$.

Очевидно, что $(0,1)$-геометрия порядка $(q-1, t)$ типа $T_{1}^{*}(\mathscr{K})$ является частичным четырехугольником порядка $(q-1,1)$. Далее, если $\mathbf{S}$ является $(0,1)$-геометрией порядка $(q-1, t)$ типа $T_{2}^{*}(\mathscr{K})$, то $\mathscr{K}$ является дугой в $\Pi_{\infty}$ и $T_{2}^{*}(\mathscr{K})$ удовлетворяет условию $(*)$ тогда и только тогда, когда $|\mathscr{K}|=q+1$ или $q+2$. 
2. Пусть $\overline{\mathbf{S}}$ - обобщенный четырехугольник, вложимьй в проективное пространство $P G(n, q)$ (отсюда $\overline{\mathbf{S}}$ - классический). Выберем точку $p$, не лежашую в гиперплоскости П из $P G(n, q)$. Пусть $P_{1}$ - проекция точечного множества $\overline{\mathbf{S}}$ на П относительно $p$, и $P_{2}$ - множество точек из П, лежаших на касательных к $\overline{\mathbf{S}}$, проходящих через $p$. Пусть $\mathbf{S}$ будет геометрией с точечным множеством $P=P_{1}-P_{2}$, множеством прямых из П, пересекаюших $P_{2}$ по крайней мере по двум точкам, и инцидентностью как в проективном пространстве. Если исходньй обобшенный четырехугольник является $Q^{-}(5, q)$ или $H_{4}\left(q^{2}\right)$, то получаются получастичные геометрии. Если $p \in \overline{\mathbf{S}}$, то возникают $T_{3}^{*}(\mathscr{O})$, где $\mathscr{O}$ - эллиптическая квадрика в $P G(3, q)$, в ортогональном случае; и $T_{2}^{*}(\mathscr{U})$, где $\mathscr{U}$ является эрмитовой униталью в $P G\left(2, q^{2}\right),-$ в унитарном. Если же $p \notin \overline{\mathbf{S}}$, то получаются новые геометрии с параметрами $s=q-1, t=q^{2}, \alpha=2$, $\mu=2 q(q-1)$ в ортогональном случае, $s=q^{2}-1, t=q^{3}, \alpha=q+1, \mu=q(q+1)\left(q^{2}-1\right)$ - в унитарном (Тас, частное сообшение).

3. Имеется еше одна конструкция для получения частичных четырехугольников из обобщенных четырехугольников.

ПреДЛОЖЕнИЕ 1.4 (Махнев). Пусть Г - псевдогеометрический граф для частичной геометрии $p G_{\alpha}(s, t)$, содержащий коклику $\mathscr{O}$, достигающую гранииу Хоффмана и граничу Цветковича для коклик. Тогда $\alpha=1, t=s^{2}-s$ и $\Sigma=\Gamma-\mathscr{O}$ является сильно регулярныцм графом с параметрами $\left(s^{2} t+s,(s-1)(t+1), s-2,(s-1)^{2}\right)$.

ДОКАЗАТЕЛЬСТВО. По Условию $|\mathscr{O}|=1+s t / \alpha=\frac{s(s-\alpha+1)(s t+\alpha)}{\alpha(s+t+1-\alpha)}$, поэтому $t=(s-1)(s+1-\alpha)$. По теореме 0.2 граф $\Sigma$ сильно регулярен. Если $L$ является $(s+1)$-кликой из $\Gamma$, то $L$ пересекает овоид $\mathscr{O}$ в единственной точке $x$. Тогда любая точка из $\mathscr{O}-\{x\}$ смежна с $\alpha$ вершинами из $L$, поэтому любая пара вершин из $L-\mathscr{O}$ смежна с $1+(\alpha-1)(s+1-\alpha)$ точками из $\mathscr{O}$. Так как граф $\Sigma$ сильно регулярен, то любое ребро из $\Sigma$ смежно с $1+(\alpha-1)(s+1-\alpha)$ точками из $\mathscr{O}$. Отсюда получаются выражения для параметров $v=s(1+s t / \alpha), k=(s-1)(t+1), \lambda=(s-2)(1+(\alpha-1)(s+1-\alpha))$. Из прямоугольного соотношения $k(k-\lambda-1)=(v-k-1) \mu$ получим выражение $\mu=s^{2}-\alpha s-s+2 \alpha-1$. Поэтому для любых несмежных вершин $a, b$ из $\Sigma$ подграф $[a] \cap[b]$ содержит $\alpha(t+1)-\mu=s+(s-1)(\alpha-1)(s+1-\alpha)=s+(\alpha-1) t$ вершин из $\mathscr{O}$.

Допустим, что $\alpha>1$. Так как каждая вершина вне $\mathscr{O}$ смежна с $t+1$ точками из $\mathscr{O}$, то $s+(\alpha-1) t \leqslant t+1$, поэтому $\alpha=2, s=1$ и $t=0$, противоречие. Отсюда $\alpha=1, \Gamma-$ псевдогеометрический граф для обобщенного четырехугольника $G Q\left(s, s^{2}-s\right)$, граф $\Sigma$ имеет параметры $\left(s^{2} t+s,(s-1)(t+1), s-2,(s-1)^{2}\right)$, собственные значения графа $\Sigma$ равны $(s-1)(t+1), s-1,-\left(s^{2}-2 s+2\right)$ и имеют кратности $1,(s-1)(s t+1), s t$ соответственно. Предложение доказано.

Таким образом, если $\mathscr{O}$ - овоид обобщенного четырехугольника $\mathbf{S}=(P, B, I)$ порядка $\left(s, s^{2}-s\right)$, то геометрия $\mathbf{S}_{\mathscr{O}}$ с множеством точек $P-\mathscr{O}$ и множеством прямых $\{L-\mathscr{O} \mid L \in B\}$ является частичным четырехугольником с параметрами $\left(s-1, s^{2}-s\right.$, $\left.(s-1)^{2}\right)$.

При $s=2$ мы получим таким путем граф Петерсена из $G Q(2,2)$. Если $s=3$, то соответствуюшие сильно регулярные графы с параметрами $(76,21,2,7)$ и $(57,14,1,4)$ не существуют (Хэмерс [55] и независимо Махнев [84], анонсировано в [75]; Вилбьринк 
и Броувер [127]). Для $s \geqslant 4$ вопрос о существовании как $G Q\left(s, s^{2}-s\right)$, так и $P Q\left(s-1, s^{2}-s,(s-1)^{2}\right)$ остается открытым. Однако имеет место

ТЕОРема 1.11 (Махнев, не опубликовано). Пусть псевдогеометрический граф для обобщенного четырехугольника $G Q(4,12)$ содержит 49-коклику. Тогда $\Gamma$ является точечным графом обобщенного четырехугольника.

4. Построение получастичных геометрий с помощью подчетырехугольников из обобщенньхх четырехугольников. Розеткой с базисной точкой $x$ в обобщенном четырехугольнике $\mathcal{S}$ порядка $(s, t)$ назьвается семейство овоидов $\mathscr{R}$, попарно пересекаюшихся в точке $x$, такое, что $\{O-\{x\} \mid O \in \mathscr{R}\}$ образует расщепление $P-x^{\perp}$.

Следуюшие результаты получены Брауном [6]. Пусть обобшенньй четырехугольник $\mathcal{S}=(P, B)$ порядка $\left(r, r^{2}\right)$ содержит подчетырехугольник $\mathcal{S}^{\prime}=\left(P^{\prime}, B^{\prime}\right)$ порядка $(r, r)$ и $a \in P-P^{\prime}$. Тогда множество точек из $P^{\prime}$, коллинеарных $a$, образует овоид в $\mathcal{S}^{\prime}$, назьваемый овоидом $O_{a}$, стянутым точкой $a$, или просто стянутым. Далее, каждая прямая из $B$ либо принадлежит $B^{\prime}$, либо является касательной к $P^{\prime}$. Для данной касательной прямой $l$ множество овоидов, стянутых точками из $l-P^{\prime}$, образует розетку $\mathcal{S}^{\prime}$, назьваемую розеткой, стянутой прямой $l$, или просто стянутой.

Оказывается, что каждый стянутьй овоид стянут не более чем двумя точками. Если овоид $O$ стянут двумя точками $x, x^{\prime}$, то определяется размер пересечения $O$ со стянутыми овоидами: $\left|O \cap O_{y}\right|$ равен 1 , если $y$ коллинеарна $x$ или $x^{\prime}$, равен $r+1$ в противном случае.

Овоид, стянутый двумя точками, назовем дважды стянутым. Если каждый стянутый овоид из $\mathcal{S}^{\prime}$ дважды стянут, то $\operatorname{Aut}(\mathcal{S})$ содержит инволюцию, фиксируюшую $\mathcal{S}^{\prime}$ поточечно, и обратно. Далее, в этом случае для любого флага $\left(x, O_{a}\right)$ имеется единственная розетка, содержашая $O_{a}$, с базисной точкой $x$.

ТеОрема 1.12 (Браун [6]). Пусть обобщенный четырехугольник $\mathcal{S}$ порядка $\left(r, r^{2}\right)$ содержит подчетырехугольник $\mathcal{S}^{\prime}$ порядка $(r, r)$ такой, что каждый стянутый овоид из $\mathcal{S}^{\prime}$ дважды стянут. Тогда система инцидентности $\mathscr{T}_{\mathcal{S}}$, точками которой являются стянутые овоиды из $\mathcal{S}^{\prime}$, а прямыци - стянутые розетки, является получастичной геометрией с параметрами $s=r-1, t=r^{2}, \alpha=2$, $\mu=2 r(r-1)$.

Пусть обобщенные четырехугольники $\mathcal{S}$ и $\mathscr{W}$ порядка $\left(r, r^{2}\right)$ содержат подчетырехугольники $\mathcal{S}^{\prime}$ и $\mathscr{W}^{\prime}$ порядка $(r, r)$ такие, что каждый стянутый овоид из $\mathcal{S}^{\prime}$ и $\mathscr{W}^{\prime}$ дважды стянут. Геометрии $\mathscr{T}_{\mathcal{S}}$ и $\mathscr{T}_{\mathscr{W}}$ изоморфньи тогда и только тогда, когда обобщенный четырехугольник $\mathcal{S}^{\prime}$ изоморфен 'W'.

В заключительном разделе доказано, что частичная геометрия, построенная по подчетырехугольнику порядка $q$ из обобщенного четырехугольника порядка $\left(q, q^{2}\right)$, двойственного к обобшенному четырехугольнику Кантора, не изоморфна известным ранее геометриям.

1.5.5. Получастичные геометрии и $S P G$ полуквадрики. Тасом [116] предложен новый метод для построения получастичных геометрий. $S P G$ полуквадрикой называется множество $R$ из $m$-мерных подпространств $P G^{1}(m, q), \ldots, P G^{r}(m, q)$ $(r>1)$ проективного пространства $P G(n, q)$, удовлетворяюшее условиям:

(1) пересечение двух различных подпространств $P G^{i}(m, q) \cap P G^{j}(m, q)$ пусто; 
(2) если подпространство $P G(m+1, q)$ содержит $P G^{i}(m, q)$, то оно имеет общую точку с 0 или $\alpha$ элементами из $R-\left\{P G^{i}(m, q)\right\}$, подпространство $P G(m+1, q)$, содержащее $P G^{i}(m, q)$ и не пересекающее элементы из $R-\left\{P G^{i}(m, q)\right\}$, назьвается касательньм к $R$ в точке $P G^{i}(m, q)$;

(3) если точка $x$ из $P G(n, q)$ не содержится ни в одном элементе из $R$, то она содержится в $\theta$ касательных к $R$ подпространствах, $\theta \geqslant 0$.

Рассмотрев $(m+1)$-мерные подпространства, содержащие $P G^{i}(m, q)$, получим, что $\alpha(q-1)$ делит $(r-1)\left(q^{m+1}-1\right)$, а число касательных к $R$ подпространств в точке $P G^{i}(m, q)$ равно

$$
\frac{q^{n-m}-1}{q-1}-\frac{(r-1)\left(q^{m+1}-1\right)}{\alpha(q-1)} .
$$

Сосчитав число флагов $(M, x)$, где $M$ - касательное к $R$ подпространство, а точка $x$ из $M$ не содержится ни в одном элементе из $R$, получим

$$
\theta=\frac{r q^{m+1}\left(\alpha\left(q^{n-m}-1\right)-(r-1)\left(q^{m+1}-1\right)\right)}{\alpha\left(\left(q^{n+1}-1\right)-r\left(q^{m+1}-1\right)\right)}
$$

Заметим, что $n \geqslant 2 m+1$. В случае равенства касательных подпространств нет и $\alpha=$ $r-1$. Если $n=2 m+2$, то любые два подпространства, касающиеся $R$ в различных точках, пересекаются.

Для данной $S P G$ полуквадрики $R$ можно построить получастичную геометрию $\mathbf{S}(R)$ следуюшим образом. Вложим $P G(n, q)$ в $P G(n+1, q)$, и пусть множество точек равно $P G(n+1, q)-P G(n, q)$, множество прямых состоит из $(m+1)$-мерных подпространств, содержащих элемент из $R$, но не лежащих в $P G(n, q)$. Тогда $\mathbf{S}(R)$ является получастичной геометрией с параметрами $s=q^{m+1}-1, t=r-1, \alpha=\alpha, \mu=(r-\theta) \alpha$.

Эта геометрия будет частичной, только если $\theta=0$. В этом случае $\mathbf{S}(R)$ будет сетью порядка $s+1=q^{m+1}$ и степени $t+1=r$.

Полярные пространства и $S P G$ полуквадрики. Спред $R$ невырожденной эллиптической квадрики $Q^{-}(2 m+3, q)(m \geqslant 0)$ содержит $q^{m+2}+1$ элементов (размерности $m$ ) и является $S P G$ полуквадрикой. Соответствующая получастичная геометрия имеет параметры $s=q^{m+1}-1, t=q^{m+2}, \alpha=q^{m}, \mu=q^{m+1}\left(q^{m+1}-1\right)$. При $m=0$ получается частичный четырехугольник $T_{3}^{*}(\mathscr{O})$. Если $m=1$ и спред $R$ для $Q^{-}(5, q)$ регулярен, то получается получастичная геометрия $T_{2}^{*}(\mathscr{U})$. Если же спред не регулярен, то получается получастичная геометрия, не изоморфная $T_{2}^{*}(\mathscr{U})$. Пусть $m>1$. Если $q$ четно, то квадрика $Q^{-}(2 m+3, q)$ имеет спред. Если же $q$ нечетно, то существование спреда $Q^{-}(2 m+3, q)$ неизвестно.

Если невырожденная квадрика $Q(2 m+2, q)(m \geqslant 0)$ имеет спред, то он не является $S P G$ полуквадрикой.

Если $R$ - спред невырожденной гиперболической квадрики $Q^{+}(2 m+1, q)(m \geqslant 1)$, то необходимо $R$ является $S P G$ полуквадрикой, но соответствуюшая получастичная геометрия является сетью.

Если невырожденное эрмитово многообразие $H\left(n, q^{2}\right)$ в $P G\left(n, q^{2}\right)$ имеет спред $R$, то $n$ четно. В этом случае $R$ содержит $q^{n+1}+1$ элементов (размерности $m=n / 2-1$ ) и является $S P G$ полуквадрикой. Соответствующая получастичная геометрия имеет 
параметры $s=q^{n}-1, t=q^{n+1}, \alpha=q^{n-1}, \mu=q^{n}\left(q^{n}-1\right)$. Однако если $n=2$, то эта геометрия совпадает с $T_{2}^{*}(\mathscr{U})$. Если же $n>2$, то существование спреда не известно.

1.5.6. Характеризационные теоремы для получастичных геометрий. Характеризации по параметрам.

Теорема 1.13 (Дебре и Тас [40]). Если $\mathbf{S}$ - истинная получастичная геометрия $c \alpha=t$, mo $\mathbf{S}=\overline{M(t+1)} u s=t, \mu=\alpha^{2} u t \in\{1,2,6,56\}$.

Теорема 1.14 (Дебре [38], Вильбринк и Броувер [128]). Пусть $\mathbf{S}-$ истинная получастичная геометрия с $\mu=\alpha^{2}$.

(1) $E$ слли $\alpha=2$, mo $\mathbf{S} \simeq U_{2,3}(n)$.

(2) $E$ сли $2<\alpha=s$, mo $\alpha=t u \mathbf{S} \simeq \overline{M(t+1)}$.

(3) Если $2<\alpha<s$, mo $\mathbf{S} \simeq L P(n, q)$.

Теорема 1.15 (Дебре [38]). Если $\mathbf{S}$ - истинная получастичная геометрия $c$ $\mu=\alpha(\alpha+1)$, удовлетворяющая аксиоме $(\mathscr{D})$, то $\mathbf{S} \simeq H_{q}^{(n+1) *}$.

\section{Вложения в проективные и аффинные пространства.}

ТЕОРема 1.16. Если $\mathbf{S}$ - истинная получастичная геометрия с параметрами $s, t, \alpha, \mu$, вложенная в $P G(n, s)(s>2)$, но не вложимая в $P G(m, s)$ для $m<n$, то п нечетно и $\mathbf{S} \simeq \overline{W(n, s)}$.

Эта теорема получена Дебре и Тасом [41] для $n=3$ и Тасом и др. [118] для $n>3$. Если $\mathbf{S}$ - получастичная геометрия с $s=\alpha=2$, то $\mathbf{S}$ является котреугольным пространством, и все такие пространства классифицированы (см. теорему 1.19).

ТеОрема 1.17. Если $\mathbf{S}^{D}$ - двойственная получастичная геометрия $с \alpha>1$, вложенная в $P G(n, s)(n \geqslant 3)$, но не вложимая в $P G(m, s)$ для $m<n$, mо $n=3$ u $\mathbf{S}^{D}$ совпадает либо со схемой точек и прямых $P G(3, q)$, либо с $H_{s}^{3}$, либо с $N Q^{-}(3,2)$.

Пусть $\mathscr{H}$ - невырожденное эрмитово многообразие из $P G\left(3, q^{2}\right)$. Система инцидентности с точечным множеством $\mathscr{H}$ и множеством прямых, не пересекающих фиксированную прямую $L$, является двойственным частичным четырехугольником, вложенным в $P G\left(3, q^{2}\right)$. Не известно, имеются ли другие двойственные истинные частичные четырехугольники, вложимые в проективное пространство.

Вложение истинных получастичных геометрий в аффинное пространство полностью исследовано только для $n \leqslant 3$.

Теорема 1.18 (Дебре и Тас [39]). Истинная получастичная геометрия $\mathbf{S}$ не вкладывается в аффинную плоскость $A G(2, s+1) . \quad$ Если $\mathbf{S}$ вкладьвается в $A G(3, s+1)$, то либо $\mathbf{S}$ - пятиугольник, вложсеннй в $A G(3,2)$, либо представление линейно и $\mathbf{S}=T_{2}^{*}(\mathscr{U})$ или $T_{2}^{*}(\mathscr{B})$.

Кополярные пространства. Кополярным пространством (Холл [56]) называется такое частичное пространство прямых, что для каждого антифлага $(x, L)$ параметр $\alpha(x, L)$ равен 0 или $|L|-1$. Кополярное пространство называется неразложимым. если оно не является объединением двух или более кополярных пространств 
на непересекающихся точечных подмножествах. Редуцированным кополярньм пространством назовем неразложимое кополярное пространство с редуцированным точечным графом. Заметим, что получастичная геометрия с параметрами $s, t, \alpha=s$ является кополярным пространством порядка $(s, t)$. В частности, двойственные сети являются кополярными пространствами. Далее мы будем допускать, что сушествует хотя бы один антифлаг $(x, L)$ с $\alpha(x, L)=0$. Холл [56] классифицировал конечные редуцированные кополярные пространства порядка $(s, t)$ с $s \geqslant 2$. Оказалось, что они являются истинными получастичными геометриями.

Теорема 1.19 (Холл [56]). Пусть $\mathbf{S}$ - конечное редуиированное кополярное пространство порядка $(s, t)$ c $s \geqslant 2$. Тогда $\mathbf{S}$ изоморфно одной из следующих получастичньх геометрий:

(1) $\overline{M(k)}, k \in\{2,3,7,57\}$;

(2) $U_{2,3}(n)$;

(3) $\overline{W(2 n+1, q)}$;

(4) $N Q^{ \pm}(2 n-1,2)$.

Кополярные пространства порядка $(2, t)$ (котреугольные пространства) были классифицированы Шультом [109], ранняя версия этого результата получена Зейделем [107].

\section{$\S$ 2. Диаграммные геометрии}

\section{1. Определения.}

2.1.1. Геометрии над множествами. Следуюшие определения предложил Титс [122]. Пусть $I$ - конечное множество. Предгеометрия над $I$-это тройка $\Gamma=$ $(X, *, t)$, где $X$ - множество объектов, $t$ - типовая функция, отображающая $X$ в $I$ (сопоставляющая объекту из $X$ его тип), и $*$ - рефлексивное симметричное отношение инцидентности на $X^{2}$, причем объекты $a, b$ одного типа инцидентны, только если $a=b$. Другими словами, $(X, *)$ - многодольньй граф с долями $t^{-1}(i), i \in I$. Флаг предгеометрии - это набор попарно инцидентных элементов. Если $T$ - флаг, то ограничение $t$ на $T$ - инъективное отображение $T$ в $I$ и $t(T)$ называется типом флага $T, I-t(T)$ назьвается котипом флага $T$. Ранг и коранг флага $T$-это $|T|$ и $|I-t(T)|$ соответственно. Камерой назьвается флаг типа $I$. Две камеры назьваются $i$-смежмными, $i \in I$, если их элементы типов, отличных от $i$, совпадают. Предгеометрия Г называется геометрией ( $I$-геометрией), если $t$ сюръективно, а каж дьй максимальньй (по включению) флаг является камерой. Геометрия называется прочной (firm) (соответственно толстой, тонкой), если каждый флаг коранга 1 содержится по крайней мере в двух (по крайней мере в трех, точно в двух) камерах.

$B$ ичет $\Gamma_{F}$ флага $F$ предгеометрии $\Gamma$ - это множество объектов $X_{F}=\{u \in X-F \mid$ $u * f$ для всех $f \in F\}$, рассмотренное как предгеометрия над $I-t(F)$. Каждый вычет геометрии сам является геометрией. Свойство предгеометрии Г называется наследственнылм, если оно выполняется во всех ее вычетах. Свойство предгеометрии Г назьвается локальным, если оно выполняется во всех ее вычетах ранга 2.

Предгеометрия $\Gamma=(X, *, t)$ связна, если граф̆ $(X, *)$ не пуст и связен. Предгеометрия Г вычетно связна, если для любого подмножества $J \subseteq I$ мощности по крайней мере 2 и каждого флага $F$ типа $I-J$ граф $\left(X_{F}, *_{F}\right)$ связен. 
2.1.2. Морфизмы, накрытия и частные. Пусть $\Gamma=(X, t, *)$ - предгеометрия над $I, \Gamma^{\prime}=\left(X^{\prime}, t^{\prime}, *^{\prime}\right)$ - предгеометрия над $I^{\prime}$. Морфизм из $\Gamma$ в $\Gamma^{\prime}$ - это отображение $\alpha: X \rightarrow X^{\prime}$ такое, что $x * y$ влечет $\alpha(x) *^{\prime} \alpha(y)$, и $t(x)=t(y)$ тогда и только тогда, когда $t^{\prime}(\alpha(x))=t^{\prime}(\alpha(y))$. Образ флага под действием морфизма является флагом. Если $I=I^{\prime}$, то морфизм $\alpha$ из Г в $\Gamma^{\prime}$ с условием $t(x)=t(\alpha(x))$ называется гомоморфизмом. Биективный морфизм $\alpha$ такой, что $\alpha^{-1}$ - морфизм, называется корреляцией. Если $\alpha$ - гомоморфизм и коррелящия, то $\alpha$ называется изоморфизмом.

Группа $G$ автоморфизмов геометрии Г называется флаг-транзитивной, если она транзитивна на флагах типа $J$ для любого $J \subseteq I$. Геометрия Г назьвается расиирением геометрии $\Gamma^{\prime}$, если она содержит флаг, чей вычет изоморфен $\Gamma^{\prime}$.

Предгеометрия $\bar{\Gamma}$ называется накрытием предгеометрии $\Gamma$, если сушествует эпиморфизм $\alpha$ такой, что для любого элемента $x$ геометрии $\bar{\Gamma}$ ограничение $\alpha$ на вычет $\bar{\Gamma}_{x}$ является изоморфизмомн на $\Gamma_{x}$. Геометрия Г просто связна (вычетно просто связна), если каждое связное накрытие Г изоморфно Г (любой вычет флага, имеюший ранг не меньше 3 , просто связен).

Геометрия $\bar{\Gamma}$ называется $m$-накрытием геометрии $\Gamma$, если сушествует эпиморфизм $\alpha$ такой, что ограничение $\alpha$ на любой вычет ранга $m$ геометрии $\bar{\Gamma}$ является изоморфизмом. В этом случае геометрия Г назьвается $m$-частным геометрии $\bar{\Gamma}$.

Универсальным $m$-накрытием геометрии Г называется такое $m$-накрытие $\widetilde{\Gamma}$ с эпиморфизмом $\widetilde{\alpha}$, что для любого $m$-накрытия $\bar{\Gamma}$ с эпиморфизмом $\bar{\alpha}$ геометрия $\widetilde{\Gamma}$ является $m$-накрытием $\bar{\Gamma}$ с эпиморфизмом $\beta$ таким, что $\widetilde{\alpha}=\bar{\alpha} \beta$.

Каждая геометрия ранга $n$ имеет универсальное $(n-1)$-накрытие.

Пусть $A$ - группа автоморфизмов предгеометрии $\Gamma=(X, *, t)$ над $I$. Определим частное $\Gamma / A$ как предгеометрию $(X / A, * / A, t / A)$ над $I$, где $X / A$ - множество орбит $A$ на $X$, две такие орбиты $A x, A y$ инцидентны (относительно $* / A$ ), если $x * y$ в $\Gamma$, и $t / A$ отображает каждую орбиту на тип ее элемента. Отображение $\pi: x \mapsto A x$ является эпиморфизмом $\Gamma$ на $\Gamma / A$, назьваемьм канонической проекиией. Следующие свойства важны для теории, развитой Титсом [124].

$(Q 1)$ Для каждого флага $F$ и его стабилизатора $A_{F}$ в $A$ каноническая проекция $\pi$ индуцирует изоморфизм $(\Gamma / F) / A_{F}$ на $(\Gamma / A) / \pi(F)$.

$\left(Q 2^{\prime}\right)$ Элементы из орбиты $A$ на $X$, инцидентные данному флагу $F$, образуют орбиту под действием стабилизатора $A_{F}$.

$\left(Q 2^{\prime \prime}\right)$ Если $x, y$ - инцидентные элементы из $Г$ и флаг $F$ инцидентен некоторому элементу из $A x$ и некоторому элементу из $A y$, то существует $a \in A$ такой, что $F$ инцидентен $a x$ и $a y$.

(Q3) В графе $(X, *)$ расстояние между двумя различными элементами, принадлежащими общей орбите под действием $A$, не меньше 4.

Если $I$ конечно, то $(Q 1)$ эквивалентно объединению $\left(Q 2^{\prime}\right)$ и $\left(Q 2^{\prime \prime}\right)$. Далее, условие $(Q 3)$ влечет $\left(Q 2^{\prime}\right)$ и $\left(Q 2^{\prime \prime}\right)$.

Если $\alpha$ - морфизм $\Gamma$ в геометрию $\Gamma^{\prime}$, то $\alpha(\Gamma)$ может не быть геометрией над множеством типов элементов в $\alpha(\Gamma)$. Мы избегаем этой ситуации следующим образом. Морфизм $\alpha$ назьвается поднимаемым, если для любого флага $F$ геометрии $Г$ и любого флага $Y$ геометрии $\Gamma^{\prime}$, содержащего $\alpha(F)$, найдется флаг $F^{\prime}$ в $\Gamma$, содержаший $F$, с $\alpha\left(F^{\prime}\right)=Y$ (флаги поднимаемы). В этом случае $\alpha(\Gamma)=(\alpha(X), \alpha(*), \alpha(t))$ - геометрия над $\alpha(I)$, в которой для $x, y \in X, i \in I$ из $t(x)=i$ следует $\alpha(i)=t^{\prime}(\alpha(x))$, а из 
$x * y$ следует $\alpha(x) \alpha(*) \alpha(y)$ и т. д. Мы назовем геометрию $\alpha(\Gamma)$ образом геометрии $\Gamma$. Если $F$ - флаг $\Gamma$, то ограничение $\alpha$ на вычет $\Gamma_{F}$ поднимается на $\alpha(\Gamma)_{\alpha(F)}$.

Вложением I-геометрии $\Gamma$ в $I^{\prime}$-геометрию $\Gamma^{\prime}$ называется корреляция $\Gamma$ на подгеометрию из $\Gamma^{\prime}$.

Пусть $\alpha$ - корреляция $I$-геометрии $\Gamma$ на $\Gamma$. Тогда $\alpha$ индуцирует подстановку $\alpha_{I}$ на $I$. Очевидно, что $\alpha_{I}$ - тождественная подстановка тогда и только тогда, когда $\alpha$ - автоморфизм. Если $\alpha_{I}$ порядка 2 , то $\alpha$ называется двойственностью, двойственность порядка 2 назьвается полярностью. Если $\alpha$ и $\alpha_{I}$ порядка 3 , то $\alpha$ назьвается тройственностью (triality). Пусть $A$ - группа, порожденная $\alpha$. Подгеометрия из $\Gamma / A$, индушированная орбитами групшы $A$, являюшимися флагами из $\Gamma$, назьвается геометрией абсолютных әлементов $\alpha$.

Примеры. (1) Геометрии абсолютных элементов полярности конечномерного линейного пространства дают нам все квадрики (в характеристике $\neq 2$ ), симплектические и унитарные пространства. Каждый абсолютный элемент является здесь вполне сингулярным подпространством, и обратно.

(2) Геометрии абсолютных элементов полярностей и тройственностей конечных классических билдингов сами являются билдингами, на которых действуют централизаторы корреляций. Все скрученные группы Шевалле получаются таким образом.

2.1.3. Геометрии смежных классов. Пусть $\Gamma=(X, t, *)$ - геометрия над $I, G$ группа автоморфизмов $\Gamma$, действуюшая транзитивно на множестве камер $(G$ флагтранзитивна). Стабилизатор $G_{F}$ флага $F$ назьвается параболической подгруппой, стабилизатор камеры называется борелевской подгруппой.

Фиксируем камеру $C=\left\{x_{i} \mid t(x)=i\right\}$ и положим $P_{i}=G_{x_{i}}$. Для любого элемента $y_{i}$ типа $i$ найдется $g \in G$ такой, что $g\left(x_{i}\right)=y_{i}$, и мы можем идентифицировать $y_{i}$ со смежным классом $g P_{i}$. Если $y_{i}$ идентифицирован с $g P_{i}$ и $z_{j}$ с $h P_{j}$, то $y_{i} * z_{j}$ тогда и только тогда, когда пересечение $g P_{i} \cap h P_{j}$ не пусто. Далее, элемент $a \in G$ переводит $g P_{i}$ в $a g P_{i}$. Отсюда геометрия $Г$ однозначно восстанавливается по группе $G$ и набору $\left\{P_{i} \mid i \in I\right\}$ ее подгрупп.

Обратно, для группы $G$ и набора $\left\{G_{i} \mid i \in I\right\}$ ее подгрупп можно определить предгеометрию $\Gamma=\Gamma\left(G,\left(G_{i}\right)_{i \in I}\right)$ над $I$. Элементы типа $i$ - это левые смежные классы $g G_{i}$. Элементы разных типов $g G_{i}, h G_{j}$ иншидентны тогда и только тогда, когда пересечение $g G_{i} \cap h G_{j}$ не пусто. Далее, группа $G$, действующая левыми сдвигами, вкладьвается в группу автоморфизмов $\Gamma$. На множестве флагов ранга 2 данного типа $G$ действует транзитивно.

(1) Действие $G$ флаг-транзитивно тогда и только тогда, когда любое семейство смежных классов $\left(x_{i} G_{i}\right)_{i \in I}$, имеюшее непустые попарные пересечения, имеет некоторый обший элемент.

Если $I=\{1,2,3\}$, то (1) эквивалентно любому из утверждений

(2) $\left(G_{1} G_{2}\right) \cap\left(G_{1} G_{3}\right)=G_{1}\left(G_{2} \cap G_{3}\right)$;

(3) $\left(G_{1} \cap G_{2}\right)\left(G_{1} \cap G_{3}\right)=G_{1} \cap\left(G_{2} G_{3}\right)$ (Титс [124]).

В общем случае $I=\{1, \ldots, n\}$ для непустого подмножества $J$ из $I$ через $m(J)$ обозначим наименьший элемент из $J$. Сейчас (1) эквивалентно утверждению 
(4) для любого $J \subseteq I$ с $|J| \geqslant 3$ верно равенство

$$
\bigcap_{j \in J-m(J)} G_{m(J)} G_{j}=G_{m(J)} \bigcap_{j \in J-m(J)} G_{j}
$$

(Бюкенхаут и Герман, не опубликовано).

Предгеометрия $\Gamma$ связна, если подгрупшы $\left(G_{i}\right)_{i \in I}$ порождают $G$. В случае флагтранзитивности геометрия Г является прочной тогда и только тогда, когда для любого $i \in I$

$$
\bigcap_{j \in J-i} G_{j} \nsubseteq G_{i}
$$

Пусть $J$-множество индексов и $\left(I_{j}\right)_{j \in J}-$ семейство множеств. Для каждого $j \in J$ пусть $\Gamma_{j}=\left(X_{j},{ }_{j}, t_{j}\right)$ является $I_{j}$-геометрией. Допустим, что множества $X_{j}, j \in J$, попарно не пересекаются, так же как и $I_{j}, j \in J$. Прямой суммой геометрий $\Gamma_{j}$ назьвается $I$-геометрия $\Gamma=(X, *, t)$, где $I=\bigcup I_{j}, X=\bigcup X_{j}$, ограничение $*(t)$ на $X_{j}$ совпадает с $*_{j}\left(\right.$ с $\left.t_{j}\right)$ и $x * y$ для $x$ и $y$, принадлежащих различным $X_{j}, X_{k}$.

2.2. Диаграммы. Геометрии ранга 2 являются строительными блоками для геометрий больших рангов. Если $\Gamma$ - геометрия над $I$, то мы можем сопоставить ей семейство вычетов $\Gamma_{i j}$ ранга 2, построенных по флагам типа $I-\{i, j\}$. Обратно, можно пытаться описать геометрии, для которых вычеты ранга 2 принадлежат некоторому классу геометрий ранга 2. Эта идея была развита в контексте билдингов Титсом [120], [121], а в общем случае Бюкенхаутом [7].

Геометрия над $I=\{0,1\}$ называется обобщенным 2-угольником, если каждый элемент типа 0 инцидентен любому элементу типа 1.

Пусть $\Gamma$ - геометрия над $I$. Различные элементы $i, j \in I$ назовем смежсными, если хотя бы один вычет типа $\{i, j\}$ не является обобшенным 2-угольником.

Базисной диаграммой $B D(\Gamma)$ назьвается граф̆ с множеством вершин $I$ и множеством ребер, состояшим из неупорядоченных пар смежных вершин (Бюкенхаут [8]). Геометрия Г над $I$ называется чистой (Пазини [98]), если для любых смежных $i, j \in I$ ни один вычет типа $\{i, j\}$ не является обобшенньм 2-угольником. В этом случае для любого флага $F$ вычет $\Gamma_{F}$ является чистой геометрией, и базисная диаграмма $B D\left(\Gamma_{F}\right)$ является индуцированным подграфом из $B D(\Gamma)$, чьи вершины - это типы элементов из $\Gamma_{F}$.

Следуюшая теорема о разложении геометрий с несвязными базисными диаграммами в прямую сумму геометрий, отвечающих связным компонентам, имеет фундаментальное значение для теории диаграмм.

ТЕОРема 2.1 (Бюкенхаут [7]). Пусть Г является въчетно-связной I-геометрией конечного ранга. Если элементы $i, j \in I$ лежат в разных компонентах связности базисной диаграммы $B D(\Gamma)$, то каждый элемент типа $i$ иниидентен любому әлементу типа $j$.

Пусть $I$ - множество. Диаграмма на $I$ - это пара $(I, f)$, где $f$ - функция, сопоставляющая любой паре $(i, j)$ элементов из $I$ класс $f(i, j)$ геометрий ранга 2 над $\{i, j\}$. Здесь $f(j, i)$ состоит из геометрий, двойственных геометриям из $f(i, j)$. 
2.3. Некоторые диаграммные геометрии ранга 2. I-геометрия Г принадлежит диаграмме $(I, f)$, если для любых $i, j \in I$ и каждого флага $F$ типа $I-\{i, j\}$ вычет $\Gamma_{F}$ принадлежит $f(i, j)$.

Пусть $Г$ является прочной вычетно-связной геометрией над $I=\{0,1\}$. Вместо $d_{p}$ и $d_{l}$ (см. введение) мы будем использовать обозначения $d_{0}$ и $d_{1}$ и назовем эти параметры 0- и 1-диаметром соответственно. Для натуральных чисел (или бесконечных) $g, d_{0}, d_{1}$ с $2 \leqslant g \leqslant \min \left\{d_{0}, d_{1}\right\}$ мы назовем $\left(g, d_{0}, d_{1}\right)$-угольником прочную вычетно-связную $\{0,1\}$-геометрию угольности $g$ с 0 - и 1 -диаметрами $d_{0}$ и $d_{1}$ (Бюкенхаут [9]). Картинка на рис. 1 представляет класс всех $\left(g, d_{0}, d_{1}\right)$-угольников.

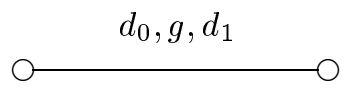

Рис. 1. $\left(g, d_{0}, d_{1}\right)$-угольник

Важньй частньй случай возникает, когда $g=d_{0}=d_{1}$. В этом случае геометрия назьвается обобщенным g-угольником (Титс [122]) и класс таких геометрий представлен на рис. 2.

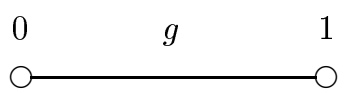

Рис. 2. Обобшенный $g$-угольник

Если $g$ равно 3,4 или 6, то мы будем использовать соответствуюшие картинки с рис. 3.

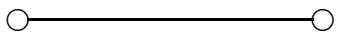

Рис. 3. Обобщенные $g$-угольники для $g=3,4$ и 6

Диаграмма $(I, f)$ называется спеииальной, если для любых двух элементов $i, j \in I$ найдутся $g_{i j}, d_{i j}, d_{j i} \in \mathbb{Z} \cup\{\infty\}$ такие, что $f(i, j)$ является $\left(g_{i j}, d_{i j}, d_{j i}\right)$-угольником. Заметим, что $g_{i j}=g_{j i}$. Специальная диаграмма $(I, f)$ назьвается диаграммой Koкстера, если для любых двух элементов $i, j \in I$ верны равенства $g_{i j}=d_{i j}=d_{j i}$.
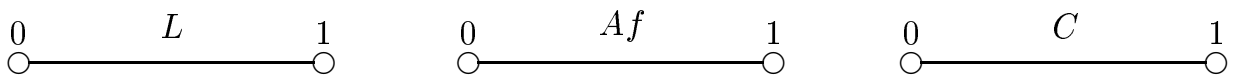

Рис. 4. Пространства прямых, аффинные плоскости и циклы

Мы используем следующие соглашения. Первая картинка на рис. 4 соответствует классу всех пространств прямых, т.е. прочных, связных $\{0,1\}$-геометрий угольности 3 , в которых любые два 0-элемента инцидентны единственному 1-элементу. Вторая картинка означает класс аффинных плоскостей, т.е. таких пространств прямых, что для любого 1-элемента $x$ и 0 -элемента $y$, находящегося на расстоянии 3 от $x$, найдется 
единственный иншидентный $y$ элемент $z$, находяшийся на расстоянии 4 от $x$. Наконец, третья картинка на рис. 4 означает класс циклов (полных графов), т.е. таких пространств прямых, что любой 1-элемент инцидентен точно двум 0-элементам.

Граф̆ Петерсена (вершины и ребра являются 0-элементами и 1-элементами соответственно) соответствует картинке с рис. 5 .

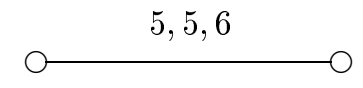

Рис. 5. Граф Петерсена

При описании конечной геометрии $\Gamma$, отвечающей диаграмме $(I, f)$, может быть полезной дополнительная информация на картинке. В частности, если для данного $i \in I$ и для любого флага $F$ котипа $i$ вычет $\Gamma_{F}$ имеет точно $q_{i}+1$ элементов независимо от $F$, то мы скажем, что $i$-порядок $\Gamma$ равен $q_{i}$. На картинке под вершиной с номером $i$ мы поставим параметр $q_{i}$. Например, рис. 6 означает цикл на $n+2$ точках.

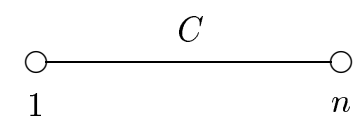

Рис. 6. Цикл на $n+2$ точках

Число, стоящее под порядком, будет означать число элементов соответствующего типа. Например, на рис. 7 помешены диаграммы, содержашие соответственно тройное накрытие обобшенного четырехугольника $G Q(2,2)$, имеющего 45 точек, 45 прямых и группу автоморфизмов 3 . Sym $(6) ;(6,5,6)$-угольник, допускающий действие группы Матье $M_{12} ;$ и $(8,6,8)$-угольник, имеющий 315 точек, 525 прямых и допускающий действие групшы $J_{2}$.

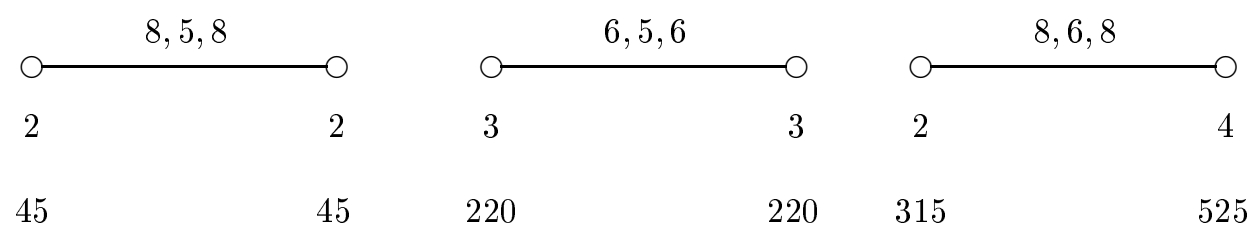

Рис. 7. Диаграммы $\left(g, d_{0}, d_{1}\right)$-угольников, допускающих действие $3 . \operatorname{Sym}(6)$, $M_{12}$ и $J_{2}$

Тройное накрытие $G Q(2,2)$ мы будем изображать более простой картинкой (см. рис. 8).

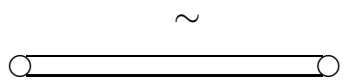

Рис. 8. Тройное накрытие $G Q(2,2)$ 
2.4. Общие результаты о диаграммных геометриях. Диаграмма, являюшаяся цепью, назьвается линейной. В работах Данщера, Шульте [27] и Шульте [104] изучались комплексы инцидентности размерности $d$. Такой комплекс является прочной вычетно-связной $I$-геометрией ранга $d$, имеюшей $i$-порядок для всех $i \in I$ и линейную базисную диаграмму. Комплекс называется регулярньм, если он имеет флагтранзитивную группу автоморфизмов.

ПроБЛЕМА 5. Для любого ли регулярного комплекса Г иниидентности ранга d существует регулярный комплекс инцидентности ранга $d+1$, в котором вычет некоторого әлемента изоморфен $Г$ ?

Прочная вычетно-связная $I$-геометрия ранга $n(n \geqslant 3)$ с линейной диаграммой, имеюшая в качестве вычетов ранга 2 пространства прямых, является пространством размерности $n$ (см. Деландшир [42]). Случай, когда правый концевой вычет является частичным пространством прямых, рассмотрен Дезой и Лорентом [48].

О результатах, связанных с билдингами, см. Шарлау [103]. Обобщения билдингов, связанные с понятием предапартамента, рассмотрены Бюкенхаутом [11].

2.5. Флаг-транзитивные диаграммные геометрии ранга 2. В пионерской работе Бюкенхаута [7] найдены флаг-транзитивные представления для 17 спорадических групп, действуюших на геометриях, имеюшие линейные диаграммы, в которых выгеты ранга 2 являются обобшенными многоугольниками или циклами.

Для диаграммы $\Delta=(A, f)$ чертой Кокстера назовем пару вершин $\{i, j\}$ такую, что $g_{i j}=d_{i j}=d_{j i}$. Сингулярной чертой назовем пару вершин $\{i, j\}$, не являюшуюся чертой Кокстера. Диаграмму $\Delta=(A, f)$ назовем диаграммой Кокстера, если каждая ее черта является чертой Кокстера.

В этом разделе мы будем предполагать, что геометрия Г принадлежит диаграмме $\Delta$ и $G$ - флаг-транзитивная группа автоморфизмов $\Gamma$. Через $s_{i}$ обозначим $i$-порядок, а через $N_{i}$ - число элементов типа $i$ геометрии Г.

2.5.1. Геометрии ранга 2 с малым дефектом. Пусть $I=\{0,1\}$. Определим $i$-дефект $\delta_{i}$ как разность $d_{i}-g$. Тогда $\delta=\left(\delta_{0}, \delta_{1}\right)$ называется дефектом $\Delta$. Особый интерес представляют диаграммы с небольшим дефектом (например, $\delta_{i} \leqslant 1$ для $i=0,1)$.

Если $\delta=(0,0)$ (диаграмма Кокстера), то по теореме Фейта-Хигмена [50] $g=3,4$, 6 или 8.

Если $\delta=(0,1)$, то по теореме 30 из [35] $g=3$ или 5. Для $g=3$ мы получим частичную геометрию $p G_{s_{0}+1}\left(s_{0}, s_{1}\right)$. В случае $g=5 \Gamma$ является графом Мура и $s_{1}=2,6$ или 56 .

ПроБлема 6. Можно ли получить обобщенную теорему Фейта-Хигмена для $\delta=(1,1)$ или даже для любого $\delta ?$

Следующие свойства могут быть использованы как дополнительные аксиомы.

$(L L)$ Любые две точки (прямые) инцидентны не более чем с одной прямой (точкой). $(M F)$ Нет двух различных точек (прямых), имеюших одинаковые вычеты.

Заметим, что $(L L)$ влечет $(M F)$. Если $s_{0}=1$ или $s_{1}=1$, то эти свойства эквивалентны. 
Пару $(\Gamma, G)$ назовем точечно геодезически транзитивной, если $G$ действует транзитивно на максимальных геодезических путях в графе инцидентности, начальные и конечные вершины которых являются точками. Аналогично определяется линейно геодезически транзитивная пара.

Пусть $d_{p} \leqslant d_{l}$. Тогда точечно геодезически транзитивная пара называется слабо геодезически транзитивной, а линейно геодезически транзитивная пара - геодезически транзитивной.

Теорема 2.2 (Бюкенхаут, Ван Мальдегем [14]). Пусть (Г, G) является геодезически транзитивной $\left(g, d_{p}, d_{l}\right)$-парой, $2 \leqslant g \leqslant d_{p} \leqslant d_{l} \leqslant g+1$. Тогда выполняется одно из следующих утверждений.

(1) Г является обобщенным многоугольником, отвечающим группе Шевалле $X_{n}(q), X_{n}(q) \leqslant G \leqslant \operatorname{Aut} X_{n}(q)$ u $X_{n}(q)=P S L(3, q), P S p(4, q), P \Omega(5, q)$, $P \Omega^{-}(6, q), \quad P S U(4, q), \quad P \Omega^{+}(4, q) \quad(c$ обобщенным четырехугольником $G Q(q, 1)), P S U(5, q), G_{2}(q),{ }^{3} D_{4}(q),{ }^{2} F_{4}(q)$.

(2) Г является геометрией вершин и ребер графа Петерсена или графа Хоффмана-Синглтона (т.е. $(5,5,6)$-угольником порядка $(1,2)$ или $(1,6)), G=$ $\operatorname{Sym}(5)$ или $U_{3}(5) \leqslant G \leqslant \operatorname{Aut} U_{3}(5)$ соответственно.

(3) Г является $(3,4,4)$-многоугольником и выполняется одно из утверждений:

(a) Г - сеть типа $(q, q)$, полученная из $P G_{2}(q)$ удалением флага $(x, l)$ и всех әлементов, инцидентных $x$ или $l, G$ содержит стабилизатор в $\operatorname{PGL}(3, q)$ флага $(x, l)$;

(b) Г является сетью $\left(H_{q}^{n+1}\right)^{D}$ порядка $q^{n}$ и степени $q+1$, G содержит подгруппу, изоморфную полупрямому произведению әлементарной группьи порядка $q^{2 n}$ и группь $\left(S L_{2}(q) \times S L_{n}(q)\right) / Z\left(S L_{2}(q) \times S L_{n}(q)\right)$, если $n>2,\left(S L_{2}(q) \times G L_{2}(q)\right) / Z\left(S L_{2}(q) \times G L_{2}(q)\right)$, ecлu $n=2, S L_{2}(2) \times \operatorname{Alt}(7)$, если $(n, q)=(4,2)$;

(c) Г двойственна геометрии из пункта (3b);

(d) Г является сетью типа $(16,9)$, чви точки являются точками аффинного пространства $A G(8,2)$, а прямые - четырехмерными аффинными подпространствами, 3-мерные подпространства которых на $\infty$ составляют 2-транзитивный спред гиперболической квадрики из $P G(7,2)$, $G$ содержит полную группу транслячий $A G(8,2)$, и ее ядро на $\infty$ изоморфно $A_{9}$;

(е) Г двойственна геометрии из пункта $(3 \mathrm{~d})$.

(4) Г является $(3,3,4)$-угольником и выполняется одно из утвержсдений:

(а) Г является пространством прямых, состоящим из точек и прямььх $P G(d, q), q \geqslant 3, u L_{d+1}(q) \leqslant G \leqslant P \Gamma L_{d+1}(q)$;

(b) Г является дезарговой аффинной плоскостью $A G(2, q), G$ содержит все транслячии, и ее ядро на $\infty$ содержит $L_{2}(q)$;

(c) $G$ действует 4-транзитивно на точках из Г, и прямые можсно отождествить с парами точек.

(5) Г является $(2,3,3)$-угольником, т.е. симметричной 2 -схемой $c \lambda>1$, u выполняется одно из утверждений: 
(a) точки геометрии Г являются точками проективного пространства $P G(d, q), d \geqslant 3$, блоки являются гиперплоскостями или их дополнениями, $L_{d+1}(q) \leqslant G \leqslant P \Gamma L_{d+1}(q): 2$ или $G=A_{7}, S_{7}((d, q)=(3,2))$;

(b) Г является единственной схемой Пәли на 11 точках, и $L_{2}(11) \leqslant$ $G \leqslant P G L_{2}(11)$

(c) Г изоморфна схеме Кантора $\mathcal{S}^{ \pm}(n)$, и $G$ содержит $2^{2 n}: S p_{2 n}(2)$ (см. Кантор [69]);

(d) Г является схемой точек и их дополнений в некотором множестве $P$, и $G$ действует 3-транзитивно на $P$.

(6) Г является обобщенным четырехугольником порядка $(1, t)$ или $(s, 1), u$ найдется почти простая группа $T_{0}$ с простьм цоколем $T$ такая, что $T \times T \leqslant G \leqslant T_{0} w r S_{2}$.

(7) Г является обобщенным 2-угольником.

Обобщенные многоугольники. Классификация всех пар $(\Gamma, G)$, где $\Gamma$ - обобщенньй $g$-угольник, а $G$ - флаг-транзитивная группа автоморфизмов, является открытой проблемой.

В случае $g=2$ вопрос эквивалентен классификации всех факторизаций, т.е. описанию всех троек $(G, A, B)$, где $A, B$ - собственные подгруппы группы $G$ и $G=A B$.

В случае $g=3$ геометрия $Г$ является проективной плоскостью порядка $n$. Если плоскость дезаргова, а $G$ не содержит $P S L(3, q)$, то либо $q=2$ и $G$ - группа Фробениуса порядка $7 \cdot 3$, либо $q=8$ и $G$-группа Фробениуса порядка $73 \cdot 9$ (Хигмэн [58]). Если же плоскость недезаргова, то $p=n^{2}+n+1$ - простое число и $G$ - группа Фробениуса порядка $\left(n^{2}+n+1\right)(n+1)$ (Кантор [72]). Более того, $n$ делится на 8 , но не является степенью 2 , и для любого делителя $d$ числа $n$ имеем $d^{n+1} \equiv 1(\bmod p)($ Фейт [49]).

В случае $g>3$ имеет место

ТЕОРема 2.3 (Зейц [108]). Пусть Г - классический обобщенный многоугольник с $g>3$ и флаг-транзитивной группой автоморфизмов $G$. Тогда либо $G-$ классическая группа автоморфизмов Г (см. утверждение (1) из заключения теоремы 2.2), либо $\Gamma=Q(4,3)$ (двойственно, $W(3))$ и $G$ - подгруппа из $2^{4} . S_{5}$, содержащая $2^{4} . S z(2)$, либо $\Gamma=Q^{-}(5,3)$ (двойственно, $\left.H(3,9)\right)$ и $G$ - расширение $P S L(3,4)$ с помощью нетривиальной подгруппь из четверной группь внешних автоморфизмов.

В первоначальной версии работы Зейца [108] были пропушены вышеуказанные спорадические примеры. Вскоре Зейц скорректировал доказательство, но не опубликовал его. Корректную версию доказательства теоремы 2.3 можно найти в работах Мейкснера [94], [95].

Для $g=4$ два спорадических примера, отвечаюших неклассическим обобщенным четырехугольникам, построены Кантором [71]. А именно, $G Q(3,5)$ с группой автоморфизмов $2^{6} . A_{6}$ и $G Q(15,17)$ с группой автоморфизмов $16^{3} .(18 \delta), \delta$ - небольшое натуральное число.

Для $g=6$ или 8 Бюкенхаут и Ван Мальдегем [14] рассмотрели точечно транзитивное действие почти простой группы, отвечающей группе из Атласа (см. Конвей и др. [24]), и доказали, что полученные пары - классические. 
Пространства прямых. Пусть $Г$ - пространство толстых прямых, в котором нет прямой, содержашей все точки. Заметим, что флаг-транзитивная группа автоморфизмов $G$ этого пространства является либо почти простой, либо аффинной, т.е. множество точек $P$ обладает структурой аффинного пространства $A G(n, p)$ надполем простого порядка $p$, а $G$ содержит группу трансляций $T$ этого пространства.

Рассмотрим сначала примеры с почти простой группой.

(1) Дезарговы проективные пространства. Здесь Г - геометрия точек и прямых дезаргова проективного пространства $P G(d, q)$ размерности $d \geqslant 2, P S L(d+1, q) \leqslant$ $G \leqslant P \Gamma L(d+1, q)$. В этом случае имеется один спорадический пример с $\Gamma=P G(3,2)$ и $G=A_{7}$ в ее дважды транзитивном действии на точках.

(2) Эрмитовы унитали. Пусть $V$ - трехмерное линейное пространство над полем $F_{q^{2}}$ с невырожденной эрмитовой формой. Эрмитова униталь $U_{H}(q)$ имеет в качестве точек $q^{3}+1$ вполне изотропных 1-подпространств, а в качестве прямых множества из $q+1$ точек, лежаших в невырожденных 2 -подпространствах. Далее, $P S U(3, q) \leqslant G \leqslant$ $P \Gamma U(3, q)$. Заметим, что при $q=2$ мы имеем аффинньй случай.

(3) Унитали Ри. Для $q=3^{2 e+1}, e \geqslant 0$, имеется группа Ри ${ }^{2} G_{2}(q)$ с дважды транзитивным действием на $q^{3}+1$ точках. Любая пара точек фиксируется единственной инволюцией, а множество точек, фиксируемых инволюцией, состоит точно из $q+1$ точек и называется прямой унитали Ри $U_{R}(q)$. Далее, ${ }^{2} G_{2}(q) \leqslant G \leqslant \operatorname{Aut}^{2} G_{2}(q)$.

(4) Пространства Витта. Пусть $q=2^{n}, n \geqslant 3$. Пространство Витта Wit $(q)$ строится по группе $P S L(2, q)$. Точками являются диэдральные подгруппы порядка $2(q+1)$, а прямыми - инволюции. При этом точка лежит на прямой, если соответствующая диэдральная подгруппа содержит инволюцию. Далее, $P S L(2, q) \leqslant G \leqslant P \Gamma L(2, q)$. Заметим, что $\operatorname{Wit}(8)$ изоморфно $U_{R}(3)$.

Примеры с аффинной группой. Пусть $G_{0}$ - стабилизатор точки. Тогда $G=$ $T G_{0}$ и $G_{0}$ - неприводимая подгруппа из $G L(n, p)$.

(1) Дезарговы аффинные пространства. Здесь $\Gamma$ - геометрия точек и прямых аффинного пространства $A G(d, q)$ с $d \geqslant 2$ и $q^{d}=p^{n}$. Если $Z$ - центр $G L(d, q)$, то ввиду флаг-транзитивности $G$ группа $Z G_{0}$ действует транзитивно на ненулевых векторах пространства. По теореме Геринга [57] выполняется одно из следующих утверждений.

(a) $G$ дважды транзитивна (и поэтому известна).

(б) $d=2, q=11$ или 23 и $G$ - одна из трех разрешпмых флаг-транзитивных групп.

(в) $d=2, q=9,11,19,29$ или $59, G_{0}^{(\infty)}=S L(2,5)$ (здесь $G_{0}^{(\infty)}$ - последний член ряда коммутантов $\left.G_{0}\right)$.

$\left(\right.$ г) $d=4, q=3$ и $G_{0}^{(\infty)}=S L(2,5)$.

(2) Недезарговы аффинные плоскости транслящий.

(a) Плоскости Люнебурга. Имеются недезарговы аффинные плоскости порядка $q^{2}$, где $q=2^{2 e+1}, e \geqslant 1$, и $^{2} B_{2}(q) \leqslant G_{0} \leqslant \operatorname{Aut}^{2} B_{2}(q)$.

(б) Плоскость Геринга порядка 27. Здесь $G_{0}=S L(2,13)$ и $G$ действует дважды транзитивно на точках плоскости.

(в) Почтиполевая плоскость порядка 9. Здесь имеется 7 возможностей для $G_{0}$.

(3) Пространства Геринга. Имеется точно два флаг-транзитивных пространства на $3^{6}$ точках с 9-точечными прямыми. В обоих случаях $G_{0}=S L(2,13)$ и $G$ действует дважды транзитивно. 
(4) Одномерньй аффинньй случай. Этот случай до конца не исследован.

(а) Аффинные плоскости трансляций. Все флаг-транзитивные аффинные плоскости, не вошедшие в пункт (2), имеют 1-мерную аффинную группу.

(б) Обобщенные системы Нетто. Пусть $q=p^{n}, p$ - нечетное простое число, и пусть $k$ - такое нечетное число, что $k(k-1)$ делит $q-1$. Через $K$ обозначим множество всех корней из 1 степени $k$ в $F_{q}$. Пусть $F_{q}$ будет множеством точек системы инцидентности $N(k, q)$, а множество прямых состоит из образов $K$ под действием группы $A G^{k-1} L(1, q)$ отображений $x \rightarrow a^{k-1} x+b$, где $a, b \in F_{q}, a \neq 0$. Эта группа и содержашие ее подгруппы из $A \Gamma^{k-1} L(1, q)$ действуют флаг-транзитивно на $N(k, q)$, однако $N(k, q)$ не всегда является пространством прямых. Следующее условие является необходимым и достаточным для того, чтобы $N(k, q)$ было пространством прямых:

$(*)$ для любого примитивного корня $\varepsilon$ степени $k$ из 1 в $F_{q}$ элементы $\varepsilon-1, \varepsilon^{2}-1$, $\ldots, \varepsilon^{k-1}-1$ лежат в разных смежных классах мультипликативной групшы $F_{q}^{*}$ по $\left(F_{q}^{*}\right)^{k-1}$.

В частности, если $k=3$, то $N(3, q)$ является пространством прямых для $q \equiv 7$ $(\bmod 12)$. Такие пространства называют обычно системами троек Нетто.

Если $k=5$, то $q \equiv 21(\bmod 40)$. Для $q<800$ имеется четыре возможности с $q=61,421,661$ и 701. Только две дезарговы проективные плоскости возникают как обобщенные системы Нетто: $N(3,7) \simeq P G(2,2)$ и $N(9,73) \simeq P G(2,8)$.

(в) Канторовский трюк раздувания (inflation trick). Пусть $L$ - прямая пространства прямых $(P, B)$ и $G_{L}$ действует флаг-транзитивно на некотором пространстве прямых $\Gamma^{\prime}$ с точечным множеством $L$. Тогда образы прямых из $\Gamma^{\prime}$ под действием $G$ образуют множество прямых флаг-транзитивного пространства прямых $\Gamma^{\prime \prime}$ с множеством точек $P$.

Стартуя, например, с $P=G F\left(q^{n}\right), L=G F(q)$ и $G=A G^{m} L\left(1, q^{n}\right)$, мы получим флаг-транзитивное пространство прямых тогда и только тогда, когда $m$ взаимно просто с $\left(q^{n}-1\right) /(q-1)$, причем в этом случае $G_{L}=A G^{m} L(1, q)$.

Если имеется обобшенная система Нетто $\Gamma=N(m+1, q)$, то мы получим новые обобщенные системы Нетто $\Gamma^{\prime \prime}=N\left(m+1, q^{n}\right)$ для значений $n$, удовлетворяющих вьшшеуказанному условию взаимной простоты.

Однако мы получим новые примеры, если найдется аффинная плоскость трансляций $\Gamma^{\prime}$ порядка $\sqrt{q}$, допускающая $A G^{m} L(1, q)$ как флаг-транзитивную группу автоморфизмов. Такие плоскости сушествуют, если $\sqrt{q}=2^{e}$ для нечетного $e \geqslant 5$ или если $\sqrt{q}$ является степенњю нечетного простого числа с показателем, не являюшимся степенњю 2 .

Теорема 2.4. Пусть $G$ - флаг-транзитивная группа автоморфизмов пространства прямых Г. Тогда выполняется одно из утверждений:

(I) $(\Gamma, G)$ является одним из примеров (1)-(4) в почти простом случае;

(II) $(\Gamma, G)$ - один из примеров (1)-(3) в аффинном случае;

(III) $\Gamma$ имеет $q=p^{a}$ точек $u G-$ подгруппа из $A \Gamma L(1, q)$;

(IV) каждая прямая имеет две точки и $G$ - любая группа, действующая дважды транзитивно на точках пространства.

В работе Пазини, Ешиары [99] имеется подробньй обзор флаг-транзитивных геометрий Бюкенхаута с большим количеством примеров. 


\section{§3. Расширения частичных геометрий}

В этом разделе рассмотрены расширения частичных геометрий, как правило, без каких-либо предположений о действии группы автоморфизмов.

Если вычет в любой точке связной геометрии $\mathbf{S}$ является $\alpha$-частичной геометрией, то $\mathbf{S}$ назьвается расширенной $(\alpha-)$ частичной геометрией, для краткости, $E p G_{\alpha}$ или $E p G$. По связности все точечные вычеты $E p G_{\alpha}$ имеют один и тот же порядок $(s, t)$, и мы будем обозначать эту геометрию как $E p G_{\alpha}(s, t)$.

Для любого антифлага $(a, L)$ геометрии $E p G_{\alpha}(s, t)$ с $\varphi(a, L) \neq 0$ выполняется неравенство $\alpha+1 \leqslant \varphi(a, L) \leqslant s+2$. Более того, если геометрия является $\varphi$-однородной с $\varphi \geqslant s+1$, то она будет и сильно однородной. Геометрия $E p G$ назьвается одноточечным расширением, если она сильно $(s+2)$-однородна; треугольной, если любые три попарно коллинеарные точки лежат в общем блоке (необходимо единственном). Геометрия $E p G_{\alpha}(s, t)$ является треугольной тогда и только тогда, когда она $(\alpha+1)$-однородна.

Геометрия $E p G_{\alpha}(s, t)$ - одноточечное расширение тогда и только тогда, когда она является 2 - $(v, k, \lambda)$-схемой с $v=1+(s+1)(1+s t / \alpha), k=s+2, \lambda=t+1$. В этом случае число ее блоков равно $b=(t+1)(\alpha+s t)(\alpha+(s+1)(\alpha+s t)) /\left(\alpha^{2}(s+2)\right)$ и $(s+2)$ делит $2 t(t+1)(2 t-\alpha)$.

Теорема 3.1 (Хобарт и Хьюз [62]). Точечный граф Г сильно $E p G_{\alpha}(s, t)$ является псевдогеометрическим для частичной геометрии $p G_{\varphi}(s+1$, $s t / \alpha)$. В частности, он сильно регулярен с параметрами $v=1+(s+1) \times$ $(1+s t(s+2)) /(\varphi \alpha), k=(s+1)(1+s t / \alpha), \lambda=s+s t(\varphi-1) / \alpha, \mu=\varphi(1+s t / \alpha) u$ собственными значениями $k, s+1-\varphi,-(1+s t / \alpha)$.

Теорема 3.2. Пусть $d$ - диаметр точечного графа геометрии $\operatorname{Ep}_{\alpha}(s, t)$, $\varphi_{0}=\min \{\varphi(a, L)\}$, где минимум берется по всем антифлагам с $\varphi(a, L) \neq 0$. Тогда справедливь оиенки:

(1) $d \leqslant \max \left\{2, s+5-2 \varphi_{0}\right\}$ (Хобарт и Хьюз [62]);

(2) если $\alpha \geqslant 2, \operatorname{mo} d \leqslant \max \left\{2,[s / 2]-\varphi_{0}+4\right\} u d \leqslant \max \{3,[(t(s+1) / 4+$ $\left.(s-\alpha)) /(t+1)]-\varphi_{0}+5\right\}$, где $[x]$ - челая часть числа $x$ (Дель Фра и Гинелли [43]).

Следуюшая теорема автора расширяет результат Камерона, Фишера [20] об s-однородных расширениях обобшенных четырехугольников.

Теорема 3.3 (Махнев [82]). Пусть $\mathbf{S}$ является s-однородной геометрией $E p G_{\alpha}(s, t), \bar{\Gamma}$ - дополнение точечного графа $\mathbf{S}$. Тогда выполняется одно из утверждений.

(1) $s=2$ и имеется точно 7 геометрий: две сильно однородные с параметрами точечного графа $(16,9,4,6)$ и $(28,15,6,10)$, еще две геометрии $c$ сильно регулярным точечным графом $(36,15,6,6)$ и $(64,27,10,12)$, а также три геометрии (по одной для $t=1,2,4)$, точечные графы которых являются графами Тәйлора (двойными антиподальными графами диаметpa 3$)$.

(2) $\mathbf{S}$ является геометрией $E p G_{2}(s, 1), \bar{\Gamma}$ - сильно регулярный граф с $\lambda=0$, $\mu=2 u \mathbf{S}-$ геометрия вериин $и$ клик $\Gamma$, соответствующих $\bar{\Gamma}(a)$ для 
$a \in \bar{\Gamma}$

(3) $\mathbf{S}$ сильно s-однородна и либо $t=\alpha u \bar{\Gamma}$ является квадратной решеткой на $(s+2)^{2}$ вершинах, либо $t=2 \alpha, s \leqslant(2 \alpha-1)(\alpha+1)^{2}, s+\alpha+1$ делит $2 s(s+1)(2 \alpha+1)$ u $\bar{\Gamma}$ является треугольным графом на $(s+2)(2 s+3)$ вериинах.

Подмножество $C$ точек геометрии $p G_{\alpha}(s, t)$ называется предовалом, если $|C| \geqslant$ $\alpha+1$ и каждая точка из $C$ соединена различными прямыми точно с $\alpha$ другими точками из $C$. Предовал $C$ с $|C|=\alpha+1$ называется суперовалом. Множество из трех точек, не лежащее на прямой, любые две точки которого коллинеарны, называется треугольником.

Суперовалы в проективной и аффинной плоскостях являются гиперовалами и афффинными овалами соответственно. Но суперовал в двойственной аффинной плоскости, т.е. проективной плоскости с удаленньми точкой и прямыми, проходящими через нее, вместе с удаленной точкой снова будет обычным гиперовалом. Поэтому суперовалы в двойственных проективной и аффиннной плоскостях сушествуют, только если плоскость имеет четный порядок.

Пусть $(a, L)$ - антифлаг геометрии $\mathbf{S}=p G_{\alpha}(s, t)$ такой, что $\varphi(a, l) \neq 0(L$ пересекает $\mathbf{S}_{a}$ ). Тогда $\mathbf{S}_{a} \cap L$ является предовалом в $\mathbf{S}_{a}$. Если геометрия $\mathbf{S}$ треугольна, то $\mathbf{S}_{a} \cap L$ - суперовал в $\mathbf{S}_{a}$ и каждый треугольник из $\mathbf{S}_{a}$ лежит в единственном суперовале $\mathbf{S}_{a} \cap L($ Хьюз [66]).

Обобщая некоторые результаты из работ Дель Фра и др. [47] и Хобарт, Хьюза [63] о расширениях геометрий с минимальным $\mu$, автор [81] получил следуюшие результаты.

TеОрема 3.4. Пусть $\mathbf{S}$ является $\varphi$-однородной $E p G_{\alpha}(s, t), \varphi \leqslant s,(a, B)-$ антифлаг с $\varphi(a, b) \neq 0$, и $b, c-$ - различнве точки из $B-\mathbf{S}_{a}$ такие, что $\mu(a, b)=$ $\mu(a, c)=\varphi(1+t(\varphi-1) / \alpha)$. Тогда $\mathbf{S}$ может бить только одной из геометрий:

(1) треугольным расширением $G Q(s, t)$ c $s \geqslant t$;

(2) геометрией $E G Q(25,5)$ с $\varphi=6$;

(3) расииренной сетью $E p G_{t}(s, t), t \leqslant 2$;

(4) расширенной двойственной 2 -схемой $\operatorname{Ep}_{2}(s, 1)$.

СлЕДСТВИЕ. Пусть $\mathbf{S}$ является $\varphi$-однородной $\operatorname{Ep}_{\alpha}(s, t), \varphi \leqslant s$, с сильно регулярным точечным графом $\Gamma$, в котором $\mu=\varphi(1+t(\varphi-1) / \alpha)$. Тогда выполняется одно из утвержсдении:

(1) $\mathbf{S}$ - треугольное расширение $G Q(t, t), t=2,3,4,8,13$;

(2) $\alpha=t=1, \varphi=2$ и Г либо совпадает с частным графа Джсонсона $J(10,5)$, либо является дополнительным графом к квадратной решетке на 16 вершинах;

(3) $\mathbf{S}$ - геометрия вериин и максимальных клик графа, дополнительного $к$ графу с $\lambda=0, \mu=2$.

Подмножество точек $K$ частичной геометрии $p G_{\alpha}(s, t)$ назьвается $(0, n)$-множеством, если каждая прямая, пересекающая $K$, пересекает $K$ по $n$ точкам. Если, кроме того, каждая прямая пересекает $K$, то $K$ называется $n$-овоидом. 
Для непустого подмножества точек $K$ частичной геометрии $p G_{\alpha}(s, t)$ через $L^{+}$ $\left(L^{-}\right)$обозначим множество прямых, (не) пересекающих $K$, и рассмотрим натуральное число $n=\min _{l \in L^{+}}|l \cap K|$. Тогда для $k=|K|$ вьполняются следуюшие нестрогие неравенства (Де Клерк, Дель Фра и Гинелли [32]):

(1) $k \geqslant n[(n-1) t+\alpha] / \alpha$, причем равенство достигается тогда и только тогда, ког да множество прямых геометрии индуцирует на $K$ подгеометрию $p G_{\alpha}(n-1, t)$ (в частности, $K$ будет $(0, n)$-множеством);

$\left(1^{\prime}\right)$ если $L^{-}$пусто, то $k \geqslant n[s t+\alpha] / \alpha$;

(2) $k \geqslant(s+1)[n(t+1)-(s+t+1-\alpha)] / \alpha$, причем равенство достигается тогда и только тогда, когда $K$ является регулярньм $(0, n)$-множеством валентности $n(t+1)-t$ и для каждой точки $P \notin K$ число прямых, проходяших через $P$ и не пересекающих $K$, равно $e=(s+t+1-\alpha) / n$.

Отметим, что граница (1) лучше (2) тогда и только тогда, когда $n<(s+t+1-\alpha) / t$. Эти гранищы совпадают, если $n=s+1$ (и $K$ является множеством всех точек геометрии) или $n=(s+t+1-\alpha) / t$. Если $n=(s+t+1-\alpha) / t$ и $k=(s+1)(s+t+1-\alpha) / t$, то $K$ является подгеометрией $p G_{\alpha}((s+1-\alpha) / t, t)$, причем для каждой точки $P \notin K$ найдется единственная прямая, проходяшая через $P$ и пересекаюшая $K$.

3.1. Расширения сетей и двойственных сетей. Небольшим обобшением расширенной двойственной сети является следуюшее понятие трансверсальной схемы. Пусть $m, c$ и $u$ - натуральные числа, большие 1 . Множество из $m c$ точек, лежаших в $m$ классах по $c$ точек, вместе с $m^{u}$ блоками порядка $c$, такое, что любой блок содержит точно одну точку из каждого класса, и любое множество из $u$ точек, выбранных в разных классах, лежит в единственном обшем блоке, называется $u$-трансверсальной схемой для $(m, c)$ или, кратко, $u$ - $T D(m, c)$-схемой.

Предположим сначала, что геометрия $\mathbf{S}$ является расширенной двойственной сетью, т.е. каждый вычет $\mathbf{S}_{a}$ есть $p G_{s}(s, t)$. В этом случае геометрия либо треугольна, либо является одноточечньм расширением. Для точки $a$ данной $t$ - $(v, k, \lambda)$-схемы $\mathbf{S}=(P, B)$ через $\mathbf{S}^{a}$ обозначим $(t-1)$-схему с множеством точек $P-\{a\}$ и множеством блоков из $B$, не инцидентных $a$ (так называемое внешнее ограничение $\mathbf{S}$ ).

ТеОрема 3.5 (Хобарт, Хьюз [62]). Пусть геометрия $\mathbf{S}$ является одноточечным расширением двойственной сети. Тогда $\mathbf{S}$ является одной из геометрий:

(1) единственная 2-(21,6,4)-схема, расширяющая двойственную аффинную плоскость порядка 4 и совпадающая с $\mathbf{M}_{22}^{a}$, әде $\mathbf{M}_{22}$ - естественная 3-(22,6,1)-схема для группьи Матье $M_{22}$;

(2) единственная биплоскость 2-(7,4,2), расширяющая двойственную аффинную плоскость порядка 2 (получаемая, как и выше, в виде внешнего ограничения 3-схемы, расширяющей проективную плоскость порядка 2).

В указанной работе [62] построен ряд примеров треугольных расширений двойственных сетей.

Теорема 3.6 (Хобарт, Хьюз [62]). Пусть геометрия $\mathbf{S}$ является одноточечным расширением сети типа $(n, m)$. Тогда

(1) $m=n+1 u \mathbf{S}-$ инверсивная плоскость порядка $n$, или

(2) $m=(n+1) / 2$ и $\mathbf{S}$ является $2-\left(n^{2}+1, n+1,(n+1) / 2\right)$-схемой. 
Далее, мы имеем $\varphi(a, L)=n+1$, поэтому в первом случае геометрия треугольна, а во втором - нет.

ПримеР 3.1. Пусть $q$ - степень нечетного простого числа, $G=P S L\left(2, q^{2}\right), H-$ подгруппа из $G$, изоморфная $P G L(2, q), G$ и $H$ естественно действуют на проективной прямой $F_{q^{2}} \cup \infty$. Пусть точками геометрии $\mathbf{S}$ являются точки этой прямой, а блоками - $G$-сопряженные с $B=F_{q} \cup \infty$ подмножества. Так как стабилизатор блока $B$ совпадает с $H$, то $\mathbf{S}$ является расширенной сетью типа (2) из заключения теоремы 3.6. (Заметим, что $\mathbf{S}$ является полуинверсивной плоскостью, так как она естественно вкладьвается в инверсивную плоскость и содержит половину блоков этой плоскости.)

До конца раздела 3.1 будем предполагать, что $\mathbf{S}$ - сильно однородная расширенная сеть, не являюшаяся одноточечным расширением. Тогда ее точечный граф Г сильно регулярен с параметрами $v=1+n+n\left(n^{2}-1\right) / \varphi, k=n^{2}, \lambda=\varphi(n-1), \mu=\varphi n$ и имеет собственные значения $n-\varphi,-n$ кратностей $n^{2}\left(n^{2}-1\right) /(\varphi(2 n-\varphi)), n+n\left(n^{2}-1\right) \times$ $(n-\varphi) /(\varphi(2 n-\varphi))$ соответственно. Поэтому $\varphi$ делит $n\left(n^{2}-1\right)$ и $\varphi(2 n-\varphi)$ делит $n^{2}\left(n^{2}-1\right)$. Заметим, что эти условия выполнены, если $\varphi=n-1, n$ или $n+1$. В случае $\varphi=n$ исследованы треугольные расширения.

Теорема 3.7 (Хобарт, Хьюз [62]). Пусть геометрия $\mathbf{S}$ - треугольное $n$-однородное расширение сети типа $(n, m)$. Тогда $\mathbf{S}$ является $3-T D(n, n+1)$-схемой $и$ каждый точечный вычет получается удалением из аффинной плоскости одного класса параллельных прямых (в частности, $n=m$ ).

Такие расширения сушествуют для любого $n$, являющегося степенью простого числа.

ПРИмеР 3.2. Пусть $q$ - степень простого числа $a, W$ есть $3 \times(q+1)$-матрица над $F_{q}$ такая, что любые ее три столбца линейно независимы (столбцы $W$ образуют овал в проективной плоскости $P G(2, q))$. Пусть точками геометрии $\mathbf{S}$ являются пары $(i, x)$, где $i$ - номер столбца $W, x \in F_{q}$; а блоками - векторы из пространства, порожденного строками $W$; пара $(i, x)$ инцидентна $w$, если $i$-я координата $w$ равна $x$.

Тогда $\mathbf{S}$ является сильно однородным треугольньм расширением сети типа $(n, n)$.

Допустим теперь, что $\varphi=n-1$. Ввиду теоремы $3.3(n-1)$-однородная расширенная сеть типа $(n, m)$ с $m \geqslant 3$ является сильно однородной.

ПримеР 3.3. Пусть $G$ - дважды транзитивная группа подстановок на множестве $\mathscr{Y}$ из $n+1$ элементов такая, что только 1 фиксирует 3 точки, и пусть $|G|=(n+1) n m$. Точками геометрии $\mathbf{S}$ являются пары $(i, j)$, где $i, j \in \mathscr{Y} ;$ а блоками - символы $[g]$, $g \in G$; пара $(i, j)$ инцидентна $g$, если $j=i g$.

Тогда $\mathbf{S}$ является сильно $(n-1)$-однородньм расширением сети типа $(n, m)$ :

(i) $\operatorname{PGL}(2, q)$ дает расширение сети типа $(q, q-1)$;

(ii) $\operatorname{PSL}(2, q)$ дает расширение сети типа $(q,(q-1) / 2)$;

(iii) $P G^{*} L\left(2, q^{2}\right), q$ нечетно, дает расширение сети типа $\left(q^{2}, q^{2}-1\right)$;

(iv) $A \Gamma L\left(2,2^{p}\right), p$ простое, дает расширение сети типа $\left(2^{p}-1, p\right)$.

Полученные геометрии треугольны в случаях (i), (iii); здесь $P G^{*} L\left(2, q^{2}\right)$ - это группа всех подстановок $K_{q^{2}}$ вида $x \rightarrow\left(a x^{\sigma_{\Delta}}+b\right) /\left(c x^{\sigma_{\Delta}}+d\right)$, где $\Delta=a b-c d \neq 0$ и $\sigma_{\Delta}=1$ 
или $q$ в зависимости от того, является $\Delta$ квадратом или нет; $A \Gamma L\left(2,2^{p}\right)$ - это группа всех подстановок $K_{2^{p}}$ вида $x \rightarrow\left(a x^{\sigma}+b\right)$, где $a \neq 0$ и $\sigma$-автоморфизм поля $F_{2^{p}}$.

Пусть, наконец, $\varphi=n-e$ с $e>1$. Тогда $n-e$ делит $e\left(e^{2}-1\right)$ и $n^{2}-e^{2}$ делит $e^{2}\left(e^{2}-1\right)$, причем $n \leqslant e^{2}$. Для максимально возможного значения $n=e^{2}$ все условия делимости вьполнены. Если $e=q$ - степень простого числа, то параметры точечного графа расширенной сети $\left(1+q^{2}+q(q+1)\left(q^{2}+1\right), q^{4},\left(q^{2}-1\right)\left(q^{2}-q\right), q^{2}\left(q^{2}-q\right)\right)$ совпадают с параметрами дополнения для графа прямых в $P G(3, q)$. Заметим, что в треугольном случае расширение сети восстанавливается по точечному графу.

Допустим теперь, что $\mathbf{S}$ является треугольным сильно однородным расширением сети типа $\left(q^{2}, q^{2}-q\right)$. Для любой точки $b$ вычета $\mathbf{S}_{a}$ имеется $q^{2}-q$ блоков, являюшихся прямыми сети $\mathbf{S}_{a}$, в то время как остальные $\left(q^{2}-1\right)\left(q^{2}-q\right)$ блоков из $b^{\perp}$ пересекают $\mathbf{S}_{a}$ по $q^{2}-q$ точкам. Если $L-$ блок сети, пересекаюший $\mathbf{S}_{a}$, но не содержаший $a$, то $L \cap \mathbf{S}_{a}$ является предовалом сети $\mathbf{S}_{a}$.

Теорема 3.8 (Хобарт, Хьюз [62]). Пусть геометрия $\mathbf{S}$ - треугольное расиирение сети типа $\left(q^{2}, q^{2}-q\right)$ и некоторый точечный вычет $\mathbf{S}_{a}$ вкладьвается в аффинную плоскость $\mathbf{A}$ порядка $q^{2}$. Тогда:

(1) если некоторый предовал $L \cap \mathbf{S}_{a}$ расширяется до овала $\mathbf{P}$, где $\mathbf{P}$ - проективная плоскость, соответствующая $\mathbf{A}$, mо $q=2$;

(2) если А дезаргова, то $q \leqslant 3$.

Исключение с $q=2$ в самом деле сушествует.

ПримеР 3.4. Пусть точками геометрии $\mathbf{S}$ будут 35 прямых $P G(3,2)$; блоками 56 спредов (множеств из 5 прямых, расщепляющих 15 точек $P G(3,2)$ ).

Тогда $\mathbf{S}$ является сильно однородным расширением сети типа $(4,2)$.

Кроме $n=e^{2}$ сушествуют спорадические возможности для $e>3$. Например:

(1) $e=4$ дает $n=6$ или 8 и в треугольном случае возможны расширения сетей типа $(6,2)$ и $(8,4)$;

(2) $e=5$ дает $n=7,10$ или 15 ;

(3) $e=6$ дает $n=8,9$ или 36 .

3.2. Расширения двойственных 2-схем. Любое расширение 2 -схем $p G_{s+1}(s, t)$ одноточечно и является предметом изучения теории блок-схем. В этом разделе мы рассмотрим расширения $\mathbf{S}$ двойственных 2-схем. Пусть $\mathbf{S}_{a}$ является двойственной 2 - $(v, k, 1)$-схемой с $r=(v-1) /(k-1)$ прямыми, проходяшими через точку, и с $b=v r / k$ прямыми. Тогда $\mathbf{S}_{a}$ является $p G_{k}(r-1, k-1)$ с $b$ точками и $v$ прямыми. Суперовал в $\mathbf{S}_{a}$ соответствует множеству $L$ из $k$ прямых в $\mathbf{S}_{a}^{D}$ такому, что любые две прямые из $L$ пересекаются и любая точка, лежашая на прямой из $L$, принадлежит точно двум прямым из $L$.

Если геометрия $\mathbf{S}$ является треугольным расширением двойственных 2 -схем и $\mathbf{S}_{a}-$ двойственная $2-(v, k, 1)$-схема с $b$ прямыми, то $k+1$ делит $b(v-k)$. Заметим, что изучение сильно $\varphi$-однородных расширений двойственных сетей $p G_{t+1}(s, t)$ равносильно изучению сильно $\varphi$-однородных частичных $(t+1,2)$-геометрий с $s+2$ точками в блоке. В частности, блочные графы таких расширений сильно регулярны (см. [65]). 
Рассмотрим сначала одноточечные расширения. Несложно понять, что одноточечное расширение двойственной 2-схемы - это в точности квази-симметричная 2-схема, в которой любые два блока пересекаются по нулю или двум точкам.

ТеОРема 3.9 (Хьюз [66]). Для любого фиксированного $k>2$ существует конечное число значений $v$, для которых одноточечные расширения $\mathbf{S}$ двойственных 2-(v,k,1)-схем существуют. Более того,

(1) если $k=3$, то $\mathbf{S}$ является единственной $3-(8,4,1)$-схемой;

(2) если $k=4$, то $\mathbf{S}$ является единственной 2-(21, 6, 4)-схемой, изоморфной внешнему ограничению схемы Матье $3-(22,6,1)$;

(3) если $k=5$, то $\mathbf{S}$ является единственной 3-(22,6,1)-схемой;

(4) если $k=6$, то $v=46$ или 96 и $\mathbf{S}$ является 2-(70, 10,6)- или 2-(305, 20,6)схемой.

В пункте (3) заключения теоремы Хьюза фигурирует еше 2-(100,12,5)-схема с 375 блоками. Однако множество точек вне фиксированного блока вместе с множеством блоков, не пересекаюших его, образуют расширенную проективную плоскость порядка 10, которая по теореме Лэма и др. [74] не сушествует.

Геометрии из пунктов (1), (3) обладают трижды транзитивными группами автоморфизмов $A G L(3,2)$ и Aut $M_{22}$ соответственно. Схема из пункта (2) имеет дважды транзитивную группу автоморфизмов $P \Sigma L(3,4)$. Сушествование схем в пункте $(4)$ неизвестно.

Тривиальная геометрия $p G_{2}(s, 1)$ (двойственная к схеме пар $(s+2)$-элементного множества) имеет интересные расширения.

Любая $E p G_{2}(s, 1)$ является полубиплоскостью $(s+2)$ точками в блоке, и обратно, любая полубиплоскость является такой $E p G$.

Треугольная полубиплоскость восстанавливается по своему точечному графу, который является локально треугольным. Следуюшие примеры приведены в статье Хьюза [66].

ПримеР 3.5. (1) Пусть В является треугольной полубиплоскостью с матрицей инцидентности $B$. Тогда полубиплоскость $\mathbf{B}_{1}$ с матрицей инцидентности

$$
B_{1}=\left(\begin{array}{cc}
B & I \\
I & B^{\mathrm{T}}
\end{array}\right)
$$

треугольна.

(2) Для каждого $k \geqslant 3$ существует треугольная полубиплоскость с $v=2^{k-1}$ точками и $k$ точками в блоке.

Для построения геометрий из пункта (2) надо стартовать с треугольной полубиплоскости, являющейся 2-(4,3, 2)-схемой, и применяя конструкцию из пункта (1), получить нужное бесконечное семейство.

ПримеР 3.6. Пусть $V=V_{n}(2)$ является линейным $n$-мерным пространством над $F_{2}, D$ - такое множество из $k$ векторов, порождающее $V$, что сумма любых четырех векторов из $D$ и сумма любых шести векторов из $D$ не является нулевым вектором. Тогда геометрия с множеством точек $V$ и множеством блоков $\{D+v \mid v \in V\}$ является треугольной полубиплоскостью. 
Первый новьй пример такого вида получается при $n=6, k=8 \mathrm{c} D=\left\{0, e_{1}, \ldots, e_{6}\right.$, $\left.e_{1}+\cdots+e_{6}\right\}$, где $\left\{e_{i}\right\}-$ базис $V$. Эта геометрия имеет 64 точки и блоки порядка 8 .

Перейдем теперь к расширениям конкретных классов двойственных 2-схем, а именно, к двойственным униталям, двойственным 2-схемам, возникающим из гиперовалов проективных плоскостей, двойственным проективным и аффинным пространствам.

Первоначально униталью называли 2-схему абсолютных точек и не абсолютных прямых унитарной полярности проективной плоскости $P G\left(2, q^{2}\right)$. Сейчас под униталью порядка $q$ понимают любую $2-\left(q^{3}+1, q+1,1\right)$-схему, $q>1$.

ТЕОРема 3.10 (Хьюз [66]). Пусть $\mathbf{S}$ является треугольным расширением двойственных униталей порядка q. Тогда $q \in\{2,4,5,6,10,12,19,22,26,40,54,82,166\}$. Ни одно из этих расширений не моэсет быть сильно $(q+2)$-однородным.

Пусть $\mathbf{P}$ - проективная плоскость четного порядка $q$, содержашая гиперовал $C$. Тогда геометрия с множеством точек, не лежащих в $C$, и множеством блоков, состояшим из $q(q-1) / 2$ прямых, не пересекающих $C$, является двойственной к $2-(q(q-1) / 2$, $q / 2,1)$-схеме.

ТЕОРемА 3.11 (Хьюз [66]). Пусть $\mathbf{S}$ является треугольным расширением схем, двойственных $к 2-(q(q-1) / 2, q / 2,1)$-схемам. Тогда $q \in\{4,6,10,22\}$. Если, кроме

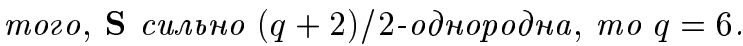

Для $q=6$ треугольное расширение с 72 точками дано далее в примере 3.7 (i) c $n=3$, а сильно 4-однородное расширение с 64 точками дано в примере 3.7 (iii).

Для $q=4$ геометрия из пункта (2) примера 3.5 с $k=6$ дает треугольное расширение схемы с 32 точками, двойственной к 2- $(6,2,1)$-схеме.

Для $k=3,4,5$ полубиплоскости с $4,8,16$ точками соответственно, данные в примере 3.5, являются единственными треугольными расширениями схем указанного вида.

ТЕОРЕМа 3.12 (Хьюз [66]). Пусть $\mathbf{S}$ является треугольным расширением двойственных 2-схем.

(1) Если $\mathbf{S}_{a}^{D}$ является 2-(v,3,1)-схемой, то $\mathbf{S}_{a}^{D}$ является геометрией точек u прямых $P G(n, 2)$.

(2) Если $\mathbf{S}_{a}^{D}$ изоморфна $P G(n, q)$, то $q=2$ или 4.

ПримеР 3.7. Пусть $\mathbf{V}=V_{N}(2)$ является многообразием Грассмана над $F_{2}$ размерности $N=n(n+1) / 2-1$, в которое двойственно вложена геометрия точек и прямых $P G(n, 2)$, т.е. точки $P G(n, 2)$ соответствуют “специальным" подпространствам $P G(n-1,2)$ из $\mathbf{V}$ и прямые $P G(n, 2)$ соответствуют точкам $\mathbf{V}$.

(i) Пусть $H$ - гиперплоскость из $P G(N+1,2)$ c $\mathbf{V}$, вложенным в $H$. Тогда геометрия с множеством точек $A G(N+1,2)=P G(N+1,2)-\mathbf{V}$ и множеством блоков, состоящим из подпространств $P G(n, 2)$, пересекающих $H$ по специальному подпространству из $\mathbf{V}$, является треугольным расширением двойственных 2-схем с вычетами, двойственными $P G(n, 2)$.

(ii) Пусть $H$ - алгебраическое многообразие из $P G(N+2,2)$ такое, что для некоторой гиперплоскости $H$, содержащей $\mathbf{V}$, алгебраическое многообразие $W \cap H$ имеет свойство: множество точек из $W \cap H$, соединенных с фиксированной точкой из $W-H$ прямьми, пересекающими $H$ в единственной точке, совпадает с $\mathbf{V}$. Тогда 
геометрия $\mathbf{S}$ с множеством точек $W-H$ и множеством блоков, состояшим из подпространств $P G(n, 2)$ из $W$, пересекающих $H$ по специальному подпространству из $\mathbf{V}$, является треугольным расширением двойственных 2-схем с вычетами, двойственными $P G(n, 2)$.

(iii) Пусть $n=3$. Тогда $N=5$ и $\mathbf{V}_{5}$ является квадрикой $Q_{5}^{+}(2)$ (без некоторых изотропных плоскостей, так как нас интересуют только “специальные” плоскости). В этом случае мы можем применить конструкцию из (iі) двумя путями. В обоих $W$ будет квадрикой $Q_{7}^{+}(2)$, вложенной в $P G(7,2)$, и $H$ - гиперплоскость из $P G(7,2)$ :

(1) Пусть $H$ - касательная гиперплоскость к $W$, тогда $W \cap H-$ конус над $Q_{5}^{+}(2)$, а вычеты $\mathbf{S}_{a}$ двойственны $P G(3,2)$. Сейчас $Q_{7}^{+}(2)$ содержит 135 точек и конус над $Q_{5}^{+}(2)$ содержит 71 точку, поэтому $\mathbf{S}$ имеет 64 точки. Эта геометрия изоморфна геометрии, построенной в (i) для $n=3$. Ее точечный граф сильно регулярен с параметрами $(64,35,18,20)$.

(2) Пусть $H$ - секушая гиперплоскость к $W$, тогда $W \cap H$ есть $Q_{6}(2)$ и выгеты $\mathbf{S}_{a}$ снова двойственны $P G(3,2)$. Так как $Q_{6}(2)$ содержит 63 точки, то $\mathbf{S}$ имеет 72 точки. Эта геометрия имеет дистанционно регулярньй точечньй граф, являющийся графом Тэйлора.

Можно показать, что если $\mathbf{S}$ - треугольное сильно однородное расшшрение двойственных геометрий к $P G(n, 2)$, то $n=3$ или 5 . Для $n=3$ пример на 64 точках является сильно однородным. Существование сильно 6-однородного расширения геометрий, двойственных $P G(5,2)$, не известно, оно должно иметь 4992 точки.

В случае $q=4$ известен единственный пример треугольного расширения $P G(n, 4)^{D}$ - это 3-схема для групшы Матье $M_{22}$, расширяюшая $P G(2,4)$. Если $n>2$, то $P G(n, 4)^{D}$ не имеет сильно 6-однородных расширений.

В работе Хайбрехт, Пазини [67] классифицированы флаг-транзитивные расширения $P G^{D}$-геометрий, где $P G^{D}$ означает геометрию прямых и точек проективного пространства. По связности геометрии точечные вычеты являются геометриями для $P G(n, q), q$ называется порядком геометрии, а $n$ - вычетной размерностью.

Если вычетная размерность $c . P G^{D}$-геометрии $S$ равна 2 , то $S$ является конечной расширенной проективной плоскостью, т.е. совпадает либо с $A G(3,2)$, либо со схемой Витта $S(22,6,3)$ для групшы Матье $M_{22}$.

Пусть $\Delta_{n+1}$ - это билдинг типа $D_{n+1}$ над $G F(2)$. Вершины диаграммы Кокстера, лежашие на конще вилки, занумеруем 0 и 2 , а их общего соседа занумеруем 1 . Выберем элемент $a$ типа 0 и через $H$ обозначим множество всех 0 -элементов, находящихся на немаксимальном расстоянии от $a$. Здесь расстояние между двумя элементами из $\Delta_{n+1}$ - это минимальная длина галереи, связывающей эти элементы. Выбросим из $\Delta_{n+1}$ все элементы $x, 0$-тени которых (0-элементы, инцидентные $x)$ содержатся в $H$. Удаляя теперь все элементы типа $i$ для $i>2$ (усекая к $\{0,1,2\}$ ), получим $c . P G^{D}$-геометрию $\Gamma_{n}$ порядка 2 и вычетной размерности $n$.

Графф коллинеарности $\Gamma_{n}$ - это графф знакопеременной формы $\operatorname{Alt}(n+1,2)$, а группа автоморфизмов $G$ является расшепляемым расширением $V \wedge V$ с помошью $L_{n+1}(2)$, где $V=V(n+1,2)$. Геометрия $\Gamma_{n}$ просто связна и не имеет фллаг-транзитивных истинных частных. 
Если $n=3$, то 0 - и 1-элементы из $\Delta_{4}$ образуют систему точек и прямых гиперболической квадрики $Q_{7}^{+}(2)$, а $H$ является касательной гиперплоскостью к этой квадрике. В качестве $H$ возьмем секушую гиперплоскость к $Q_{7}^{+}(2)$, а через $\Gamma_{3}^{\prime}$ обозначимгеометрию, полученную удалением из $\Delta_{4}$ гиперплоскости $H$ и всех элементов типа 3 . Тогда $\Gamma_{3}^{\prime}$ является $c . P G^{D}$-геометрией порядка 2 и вычетной размерности 3 с 72 точками ( $\Gamma_{3}$ имеет 64 точки).

Геометрия $\Gamma_{3}^{\prime}$ просто связна, не имеет флаг-транзитивных истинных частных, а ее группа автоморфизмов изоморфна $S p_{6}(2)$ (это стабилизатор $H$ в групе $\left.O_{8}^{+}(2)\right)$.

Теорема 3.13 (Хайбрехт, Пазини [67]). Пусть Г является флаг-транзитивной c. $P G^{D}$-геометрией въчетной размерности $\geqslant 3$. Тогда Г изоморфна $\Gamma_{n}$ или $\Gamma_{3}^{\prime}$.

О расширениях $A G(n, q)^{D}$ получены лишш частичные результаты.

ТЕОРема 3.14 (Хьюз [66]). Пусть $\mathbf{S}$ является треугольным расширением $A G(n, q)^{D}$. Тогда q четно.

ПримеР 3.8. Во вложении точек и прямых $P G(n, 2)$ как специальных подпространств и точек многообразия Грассмана $V_{N}(2)$ мы можем ограничиться вложением точек и прямых из $A G(n, 2)$ и назвать полученное многообразие $\mathbf{B}_{N}$. Вкладывая $\mathbf{B}_{N}$ в гиперплоскость $H$ из $P G(N+1,2)$, мы получим точную копию конструкции из примера 3.7 (i), являюшуюся треугольным расширением $A G(n, 2)^{D}$ для каждого $n>2$. Эта геометрия является также треугольной полубиплоскостью с блоками порядка $2^{n}$ и с $2^{N+2}$ точками.

Эта конструкция предложена Пазини.

ТЕОРЕМа 3.15 (ХЬюз [66]). Пусть $\mathbf{S}$ является одноточечным расширением двойственных 2-схем.

(1) Если $\mathbf{S}_{a}^{D}$ является униталью или проективной схемой $P G(n, q)$, то $\mathbf{S}_{a}^{D}=$ $P G(2, q), q=2$ или 4 .

(2) Если $\mathbf{S}_{a}^{D}$ является $2-(q(q-1) / 2, q / 2,1)$-схемой, то $q=4$ и $\mathbf{S}$ - биплоскость, являющаяся $2-(16,6,2)$-схемой.

(3) Если $\mathbf{S}_{a}^{D}$ является аффинной схемой $A G(n, q)$, то $n$ четно, $q=2$ и $\mathbf{S}$ является либо 2-(21,6,4)-схемой, либо биплоскостью с блоками порядка $2^{n}$. Обратно, любая биплоскость с блоками порядка $2^{n}$ является одноточечным расширением схем, двойственных $A G(n, 2)$.

3.3. Расширения обобшенных четырехугольников. В этом разделе рассматриваются расширения обобшенных четырехугольников, не предполагающие какого-либо группового действия. Результаты о расширениях обобшенных четырехугольников с флаг-транзитивной группой автоморфизмов можно найти в обзоре Камерона, Хьюза, Пазини [22].

Обобщенньй четырехугольник назовем квазиклассическим, если $\{s, t\}=\{q-1$, $q+1\}$ для некоторой степени $q$ простого числа или $s$ и $t$ являются неотрицательными степенями одного и того же простого числа. Автором [76] найден (довольно громоздкий) список допустимых параметров сильно регулярных геометрий, являющихся треугольными расширениями квазиклассических обобщенных четырехугольников. 
Теорема 3.16 (Камерон, Хьюз, Пазини [22]). Пусть $\mathbf{S}$ является расширенным обобщенным четырехугольником $E G Q(s, t), \varphi_{0}=\min \{\varphi(a, L)\}$, дде минимум берется по все антифлагам с $\varphi(a, L) \neq 0$.

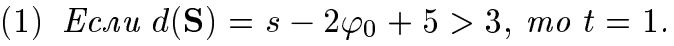

(2) Eсли $d(\mathbf{S})=s+1$, то либо $s=2$ и $\mathbf{S}$ - аффинное полярное пространство диаметра 3 , либо $t=1$ и $\mathbf{S}$ является геометрией Джонсона $\mathscr{J}_{s+1}$, либо $s=1$ u $\mathbf{S}$ - расширенная двойственная решетка.

Эти результаты уточняются в работе Дель $\Phi$ ра, Гинелли и Пазини [44]. Для $E G Q$ пусть индекс $\varphi_{0}$ определен как и вьше, $\mu=\min \{\mu(a, b) \mid d(a, b)=2\}$.

ТеОРема 3.17 (Дель Фра, Гинелли, Пазини [44]). Пусть $\mathbf{S}$ является расширенным обобщенным четырехугольником $E G Q(s, t), d$ - диаметр точечного графа, $\varphi_{0}=\min \{\varphi(a, L)\}$, где минимум берется по всем антифлагам с $\varphi(a, L) \neq 0$. Тогда справедливь оценки:

(1) $d \leqslant \max \left\{3,(s t+4 s-5 t) /(2 t+2)-2 \varphi_{0}+4\right\}$;

(2) $d \leqslant \max \left\{3, s-\varphi_{0}+5-\sqrt{\mu / t}\right\}$

(3) $d \leqslant \max \left\{3,2\left(s+1-\varphi_{0}\right)+5-4 \mu /(s t+1)\right\}$.

Некоторый прогресс достигнут в изучении расширений обобшенных четырехугольников с небольшим числом точек на прямой. Треугольные расширения $G Q(2, t)$ найдены в работе Бюкенхаута, Юбо [12]. Для каждого значения $t=1,2$ и 4 имеется единственное одноточечное расширение $G Q(2, t)$ (Тас [117]).

Теорема 3.18 (Махнев, Падучих [89]). Пусть геометрия $\mathbf{S}$ является треугольным расширением $G Q(3, t), \Gamma$ - ее точечный граф. Тогда выполняется одно из следующих утверэсдений.

(1) $t=1, \Gamma$ - либо граф Джсонсона $J(8,4)$ или его частное, либо один из двух локально $G Q(3,1)$-графов на 40 вершинах.

(2) $t=3 u \Gamma-$ граф ранга 3 для $U_{5}(2)$.

(3) $t=9$ и $Г-$ граф Маклафлина.

(4) $t=5$ и Г - граф диаметра 3 на 160 вериинах, имеющий относительно любой вериинь диаграмму, изображенную на рис. 9.

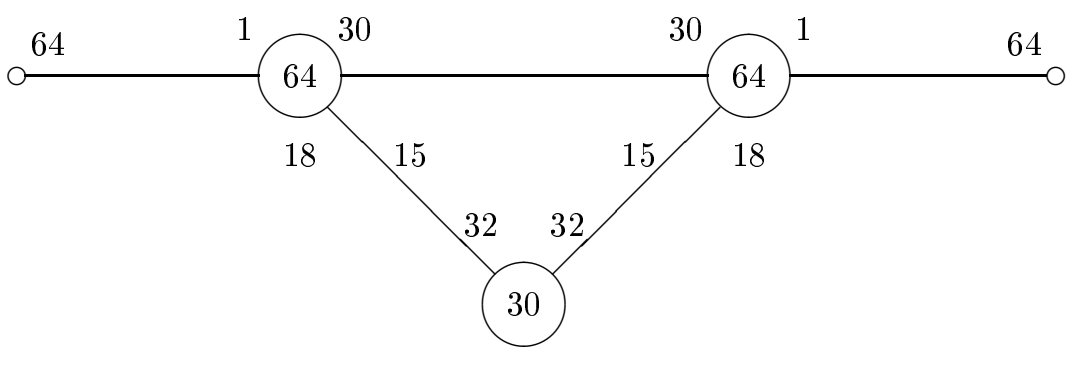

Рис. 9. Локально $G Q(3,5)$-граф 
Эта теорема является следствием результатов нескольких авторов. Во-первых, параметр $t$ равен $1,3,5$ или 9 (см. Пэйн и Тас [100]). Случай $t=1$ рассмотрен в работе Блокхейса и Броувера [2]. В работе автора [80] найдены сильно регулярные локально $G Q(3, t)$-графы. Если $t=3$, то автором [77] доказано, что Г - сильно регулярный граф̆ с параметрами $(176,40,12,8)$. Пасечник [96] с помощњю компьютерных вычислений получил тот же результат. Им же установлено, что любой сильно регулярньй локально $G Q(3,3)$-граф изоморфен графу ранга 3 для унитарной групшы $P S U(5,2)$. Пасечник [97] снова с помощью компютерных вычислений доказал, что $E G Q(3,9)$ является графом Маклафлина. Чисто комбинаторное доказательство этого факта получено автором и Падучих [90]. Наконец, случай $t=5$ рассмотрен автором [85] (анонсировано в [79]). Сушествование локально $G Q(3,5)$-графа из заключения теоремы получено Ешпарой [129], но вопрос о его единственности остается открытьм.

При исследовании локально $G Q(4, t)$-граффов особьй интерес вызывает случай $t=2$. Описание гиперовалов из $G Q(4,2)$ получено автором [83]. Подграф̆ $\Delta$ из $G Q(s, t)$ назьвается гиперовалом, если любая прямая пересекает $\Delta$ по 0 или 2 точкам.

Теорема 3.19 (Махнев, Падучих [91]). Пусть геометрия $\mathbf{S}$ является треугольным расширением $G Q(4,2)$, и ее точечный граф Г вполне регулярен. Тогда Г является одним из графов:

(1) сильно регулярный граф с параметрами $(126,45,12,18)$ на множсестве векторов нормы 1 в 6-мерном ортогональном пространстве над $G F(3)$ типа "-" с отношением смежсности, задаваемым ортогональностью, имеющий группу автоморфизмов $O_{6}^{-}(3)$;

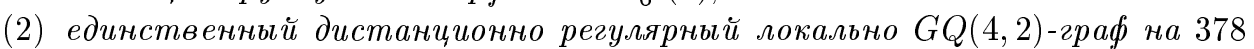
вериинах с массивом пересечений $(45,32,12,1 ; 1,6,32,45)$ (это 3-накрытие графа из пункта (1)).

3.4. Некоторые обобщения расширений. В этом разделе рассматриваются геометрии с диаграммой L.pG и элементами типов 0,1 и 2, называемыми точками, прямыми и плоскостями соответственно. Такие геометрии назьваются локально частичными геометриями.

Пространство прямых порядка $(r, s)$ является $2-(r(s+1)+1, r+1,1)$-схемой, т.е. частичной геометрией $p G_{r+1}(r, s)$. В случае $r=1$ мы получим обычную расширенную частичную геометрию. Для любой связной геометрии с диаграммой L.pG порядки элементов типов 0,1 и 2 не зависят от выбора этих элементов и обозначаются $r, s$ и $t$ соответственно. При этом геометрия обозначается как $L p G(r, s, t)$. Геометрия $L p G(r, s, t)$ является треугольной тог да и только тогда, когда она $(\alpha r+1)$-однородна.

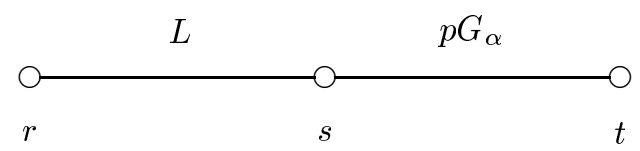

Рис. 10. L.pG-диаграмма

Теорема 3.20 (Дель Фра, Гинелли [43]). Пусть $d$ - диаметр точечного гра$\oint$ ба $L p G(r, s, t), \varphi_{0}=\min \{\varphi(a, L)\}$, где минимум берется по все антифлагам $c$ 
$\varphi(a, L) \neq 0$. Тогда справедливы оценки:

(1) $d \leqslant \max \left\{2, \min \left\{d_{1}, d_{2}, d_{3}, d_{4}\right\}\right\}$, əде

$$
\begin{array}{ll}
d_{1}=[s / 2]-\alpha+3, & d_{2}=\left[\left(r s-2 \varphi_{0}\right) /(r+1)\right]+4, \\
d_{3}=[(s-r-\alpha) / 2]+3, & d_{4}=\left[\left(r s-\varphi_{0}\right) /(r+1)\right]-r+4 ;
\end{array}
$$

(2) $d \leqslant \max \left\{3, \min \left\{d_{5}, d_{6}\right\}\right\}$, əde

$$
\begin{aligned}
& d_{5}=[(t(s+1) / 4+s-\alpha) /(t+1)]-\alpha+4, \\
& d_{6}=\left[\frac{r(t(s+1)+4(s+t+1-\alpha))}{2(r+1)(t+1)}-\frac{2 \varphi_{0}}{(r+1)}\right]+4 .
\end{aligned}
$$

Следующий пример показьвает, что граница из пункта (1) теоремы 3.20 достигается.

ПримеР 3.9. В линейном пространстве $F_{2}^{s+2}$ пусть множество точек $P$ состоит из векторов с четным числом нулевых координат; множество блоков (плоскостей) $B$ состоит из векторов с нечетным числом нулевых координат; точка инцидентна блоку, если они различаются в единственной координате; множество прямых состоит из пар точек, лежаших в обшем блоке. Тогда геометрия $\mathscr{D}_{s+2}=(P, B)$ является треугольной $E p G_{2}(s, 1)$ с диаметром $d=[s / 2]-\varphi+4$.

Дальнейшим обобшением для $E p G_{\alpha}$ является геометрия $\mathcal{S}$ со следуюшей диаграммой типа $\left(p G \cdot A_{m} \cdot p G\right)$ ранга $n=m+2, m \geqslant 1$. Как обычно, точка, прямая, плоскость, $\ldots$, двойственная прямая, двойственная точка означают элементы типов $1,2,3, \ldots$, $n-1, n$. Если $x$ - элемент $\mathcal{S}$, то тенью $x$ называется множество точек $\sigma(x)$ из $\mathcal{S}$, инцидентных с $x$. Мы можем сформулировать следующее свойство:

(Int) если $\sigma(x) \cap \sigma(y)$ не пусто для некоторых элементов $x, y$ геометрии $\mathcal{S}$, то найдется такой элемент $z$ из $\mathcal{S}$, инцидентньй $x$ и $y$, что $\sigma(z)=\sigma(x) \cap \sigma(y)$.

В статье Дель Фра, Гинелли и Пазини [45] показано, что почти все геометрии, принадлежашие диаграмме $\left(p G \cdot A_{m} . p G\right)$ с $m \geqslant 3$, удовлетворяюшие свойству (Int), известны.

Двойным $(h, k)$-усечением проективной геометрии размерности $h+n+k$ называется геометрия $\mathscr{G}$, образованная всеми $i$-мерными проективными подпространствами для $i=h, h+1, \ldots, h+n-1$. Это усечение принадлежит диаграмме $\left(L^{D} \cdot A_{n-2} . L\right)$.

Теорема 3.21 (Спрагу [112]). Геометрия, принадлежащая диаграмме $\left(L^{D}\right.$. $\left.A_{n-2} \cdot L\right)$, является двойным усечением проективной геометрии тогда и только тогда, когда она удовлетворяет свойству (Int).

В заключение этой теоремы входят и проективные геометрии как $(0,0)$-усечения.

Верхним $k$-усечением аффинной геометрии размерности $n+k$ (принадлежащей диаграмме $\left.\left(A f . A_{n+k-1}\right)\right)$ называется геометрия $\mathscr{G}$, образованная всеми $i$-мерными аффинньми подпространствами для $i=1,2, \ldots, n$. Это усечение принадлежит диаграмме $\left(A f \cdot A_{n-2} \cdot L\right)$. 
Теорема 3.22 (Дель Фра, Гинелли, Пазини [45]). Пусть геометрия $\mathscr{G}$ принадлежит диаграмме $\left(A f . A_{n-2} \cdot L\right)$. Если $n \geqslant 4$ или каждая прямая содержит более трех точек, то $\mathscr{G}$ является верхним усечением аффинной геометрии.

Нижним $k$-усечением полярного пространства ранга $n+k$ (принадлежашего диаграмме $\left.\left(C_{n+k}\right)\right)$ называется геометрия $\mathscr{G}$, образованная всеми $i$-мерными вполне сингулярными подпространствами для $i=k+1, \ldots, n+k$ (эти подпространства мы назовем точками, прямыми и т. д.). Это усечение принадлежит диаграмме $\left(L^{D} \cdot C_{n-1}\right)$.

ТЕорема 3.23 (Ронан [102]). Пусть геометрия $\mathscr{G}$ принадлежсит диаграмме $\left(L^{D} . C_{n-1}\right)$ с $n \geqslant 4$. Геометрия $\mathscr{G}$ является нижним усечением полярного пространства тогда и только тогда, когда она удовлетворяет свойству (Int).

Для данного невырожденного полярного пространства $\mathcal{S}$ ранга $n$ әиперплоскостью называется такое истинное подмножество точек $H$, что каждая прямая из $\mathcal{S}$, не содержашаяся в $H$, пересекает $H$ в единственной точке. Аффинным полярнылм пространством $\mathscr{G}$ называется геометрия, полученная удалением из $\mathcal{S}$ всех сингулярных подпространств, содержащихся в $H$ (Коэн и Шульт [23]).

Пусть $K_{H}$ - поточечный стабилизатор $H$ в группе автоморфизмов геометрии $\mathscr{G}$, $A$ - подгруппа из $K_{H}$. Тогда естественно определяется частное $\mathscr{G} / A$, назьваемое стандартным частным $\mathscr{G}$ (естественно, разрешается $A=1$ ) и принадлежашее диаграмме $\left(\right.$ Af. $\left.C_{n-1}\right)$.

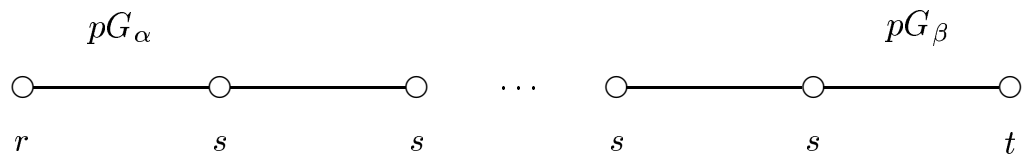

Рис. 11. $p G \cdot A_{m} \cdot p G$-диаграмма

ТЕОРема 3.24 (Кайперс и Пазини [26]). Геометрия, принадлежсащая диаграмме $\left(\right.$ Af. $\left.C_{n-1}\right)$ с $n \geqslant 3$, является стандарным частным аффинного полярного пространства тогда и только тогда, когда она удовлетворяет свойству (Int).

Имеется общая конструкция для построения различных семейств геометрий. Пусть $X, Y$ - подпространства из $P G(m, q)$ размерностей $x, y$ соответственно $(x=m$ и $y=-1$ разрешаются). Через $\left.\Gamma_{m}\right|_{Y} ^{X}$ обозначим геометрию всех истинных подпространств из $P G(m, q)$, не пересекаюших $Y$ и вместе с $X$ порождаюших $P G(m, q)$. Размерности этих подпространств и ранг $n$ геометрии принимают следуюшие значения:

(1) если $x<m$ и $y \geqslant 0$, то размерности равны $m-x-1, m-x, \ldots, m-y-2, m-y-1$ и $n=x-y+1$

(2) если $x<m$ и $y=-1$, то размерности равны $m-x-1, m-x, \ldots, m-1$ и $n=x-y$

(3) если $x=m$ и $y \geqslant 0$, то размерности равны $0,1, \ldots, m-y-1$ и $n=x-y$;

(4) если $x=m$ и $y=-1$, то размерности равны $0,1, \ldots, m-1$ и $n=x-y-1$.

В последнем случае мы имеем $P G(m, q)$. Если $x=m-1$ и $y=-1$, то $\left.\Gamma_{m}\right|_{Y} ^{X}=$ $A G(m-1, q)$.

Если $x=m$ и $y=0$, то $\left.\Gamma_{m}\right|_{Y} ^{X}$ является геометрией, двойственной к $A G(m-1, q)$, причем $Y$ является бесконечно удаленной точкой. 
Если $x<m-1$ и $y=-1$, то геометрия называется разрежсеннылм (attenuated) пространством. Эта геометрия принадлежит диаграмме $\left(N . A_{n-1}\right)$, где $N$ означает сеть $p G_{t}(s, t), s=q^{m-x}-1, t=q$.

Если $x=m$ и $y \geqslant 1$, то $\left.\Gamma_{m}\right|_{Y} ^{X}$ является геометрией, двойственной к предыдущей (двойственное разреженное пространство). Эта геометрия принадлежит диаграмме $\left(A_{n-1} \cdot N^{D}\right)$.

Если $x=m-1$ и $y=0$, то геометрия называется биаффинным пространством. Эта геометрия принадлежит диаграмме $\left(A f \cdot A_{n-2} \cdot A f^{D}\right)$.

Если $x=m-1$ и $y \geqslant 1$, то $\left.\Gamma_{m}\right|_{Y} ^{X}$ называется аффинным разрежсеннылм пространством. Эта геометрия принадлежит диаграмме $\left(A f \cdot A_{n-2} \cdot N^{D}\right)$.

Если $x<m-1$ и $y=0$, то $\left.\Gamma_{m}\right|_{Y} ^{X}$ является геометрией, двойственной к предыдущей (двойственное аффинно разреженное пространство). Эта геометрия принадлежит диаграмме $\left(N \cdot A_{n-2} \cdot A f^{D}\right)$.

Если $x<m-1$ и $y \geqslant 1$, то геометрия назьвается дважды разрежсенным пространством. Эта геометрия принадлежит диаграмме $\left(N \cdot A_{n-2} \cdot N^{D}\right)$.

Можно определить верхние усечения для разреженных пространств (двойственно, нижние усечения для двойственных разреженных пространств). Эти усечения принадлежит диаграмме $N . A_{m} \cdot L$ (двойственно, $\left(L^{D} A_{m} \cdot N^{D}\right)$ ).

Пусть невырожденное полярное пространство $\mathcal{S}$ ранга $r$ естественно вложено в $P=$ $P G(m, q)$. Пусть $X$-подпространство из $P$ коразмерности $x, 1 \leqslant x \leqslant r-2$. Построим новую геометрию $\mathcal{S} / X$, элементами которой являются все сингулярные подпространства, порождаюшие вместе с $X P G(m, q)$. Их размерности равны $x-1, x, \ldots, r-1$, а геометрия имеет ранг $n=r-x+1 \geqslant 3$, удовлетворяет свойству (Int) и выполняется одно из следующих утверждений:

(1) $x=1$, геометрия $\mathcal{S}^{/ X}$ является аффинным полярным пространством с диаграммой $\left(A f . C_{r-x}\right)$;

(2) $x>1$, геометрия $\mathcal{S} / X$ назьвается разрежсенным полярным пространством и имеет диаграмму $\left(N . C_{r-x}\right)$, где $N$ означает сеть $p G_{t}(s, t), s=q^{x}-1, t=q$.

ТЕорема 3.25 (Дель Фра, Гинелли, Пазини [44]). Пусть геометрия $\mathscr{G}$ удовлетворяет свойству (Int) и принадлежст диаграмме ( $\left.p G . A_{m} . p G\right)$ c m $\geqslant 3$ u r,s,t как в начале раздела. Тогда $\mathscr{G}$ либо является геометрией из заключения теорем $3.22-3.24$, либо принадлежит одной из диаграмм $\left(A f \cdot A_{m} \cdot N^{D}\right),\left(N \cdot A_{m} \cdot N^{D}\right)$ или $\left(N . C_{m+1}\right)$.

\section{СПИСОК ЛИТЕРАТУРЫ}

[1] Ascbacher M. The non-existence of rank three permutation groups of degree 3250 and subdegree 57 // J. Algebra. 1971. V. 19. P. 538-540.

[2] Blokhuis A., Brouwer A. E. Locally 4-by-4 grid graphs // J. Graph Theory. 1989. V. 13. P. 229-244.

[3] Bose R. Strongly regular graphs, partial geometries and partially balanced designs // Pacif. J. Math. 1963. V. 13. P. 389-419.

[4] Brown M.R. Semipartial geometries and generalized quadrangles of order $\left(r, r^{2}\right) / /$ Simon Stevin. 1998. V. 5. P. 187-205.

[5] Brouwer A., Cohen A., Neumaier A. Distance Regular Graphs. Berlin: SpringerVerlag, 1989. 
[6] Brouwer A., van Lint J. Strongly regular graphs and partial geometries // Enumeration and Design / ed. M. Jackson and S. Vanstone. New York: Academic Press, 1984. P. $85-122$.

[7] Buekenhout F. Diagrams for geometries and groups // J. Combin. Theory. Ser. A. 1979. V. 27. P. 121-151.

[8] Buekenhout F. The basic diagram of a geometry // Lecture Notes in Math. 1981. V. 893. P. 1-29.

[9] Buekenhout F. $\left(g, d^{*}, d\right)$-gons // Finite Geometries / ed. N. Johnson et al. New York: Marcel Dekker, 1983. P. 93-112.

[10] Buekenhout F. Diagram geometries for sporadic groups // Contemp. Math. 1985. V. 45. P. 132-142.

[11] Buekenhout F. Foundations of incidence geometry // Handbook of Incidence Geometry / ed. F. Buekenhout. Amsterdam: Elsevier, 1995. P. 63-105.

[12] Buekenhout F., Hubaut X. Locally polar spaces and related rank 3 groups // J. Algebra. 1977. V. 45. P. 391-434.

[13] Buekenhout F., Lefevre C. Generalized quadrangles in projective spaces // Arch. Math. 1974. V. 25. P. 540-552.

[14] Buekenhout F., Van Maldeghem H. Remarks on finite generalized hexagons and octagons with a point transitive automorphism group // Finite Geometry and Combinatorics / ed. F. De Clerck et al. Cambridge: Cambridge Univ. Press, 1993. P. 89-102.

[15] Buekenhout F., Pasini A. Finite diagram geometries extending buildings // Handbook of Incidence Geometry / ed. F. Buekenhout. Amsterdam: Elsevier, 1995. P. 1143-1254.

[16] Caldebrank A. On uniformly packed $[n, n-k, 4]$ codes over $G F(q)$ and a class of caps in $P G(k-1, q) / /$ J. London Math. Soc. 1982. V. 26. P. 365-384.

[17] Caldebrank A., Kantor W. The geometry of two-weight codes // Bull. London Math. Soc. 1986. V. 18. P. 97-122.

[18] Cameron P. Partial quadrangles // Quart. J. Math. 1974. V. 25. P. 1-13.

[19] Cameron P. Permutation Groups. Cambridge: Cambridge Univ. Press, 1999.

[20] Cameron P., Fisher P.H. Small extended generalized quadrangles // European J. Combin. 1990. V. 11. P. 403-413.

[21] Cameron P., Goethals J., Seidel J. Strongly regular graphs having strongly regular subconstituents // J. Algebra. 1978. V. 55. P. 257-280.

[22] Cameron P., Hughes D. R., Pasini A. Extended generalized quadrangles // Geom. Dedicata. 1990. V. 35. № 1-3. P. 193-228.

[23] Cohen A., Shult E. Affine polar spaces // Geom. Dedicata. 1990. V. 35. № 1-3. P. 43-76.

[24] Conway J.H., Curtis R. T., Norton S. P., Parker R. A., Wilson R. A. Atlas of Finite Groups. Oxford: Clarendon Press, 1985.

[25] Coxeter H. Twelve points in PG(5,3) with 95040 self-transformations // Proc. Roy. Soc. London A. 1958. V. 247. P. 279-293.

[26] Cuypers H., Pasini A. Locally polar geometries with affine planes // European J. Combin. 1992. V. 13. P. 39-57.

[27] Danzer L., Schulte E. Reguläre Inzidenzkomplexe I // Geom. Dedicata. 1982. V. 13. P. $295-308$.

[28] De Clerck F. The pseudo-geometric $(t, s, s-1)$-graphs // Simon Stevin. 1979. V. 53. P. 301-317.

[29] De Clerck F. New partial geometries constructed from old ones // Bull. Math. Soc. Belg. - Simon Stevin. 1998. V. 5. P. 255-263.

[30] De Clerck F., Dye R., Thas J. An infinite class of partial geometries associated with the hyperbolic quadric in $P G(4 n-1,2)$ // European J. Combin. 1980. V. 1. P. 323-326.

[31] De Clerck F., De Soete M., Gevaert H. A characterization of the partial geometry $T_{2}^{*}(K) / /$ European J. Combin. 1987. V. 8. P. 121-127.

[32] De Clerck F., Del Fra A., Ghinelli D. Pointsets in partial geometries // Advances in Finite Geometries and Designs. Proc. 3rd Isle of Thorns Conf. Chelwood Gate, UK, 1990 / ed. J. Hirshfeld et al, 1991. P. 93-110. 
[33] De Clerck F., Gevaert H., Thas J. Partial geometries and copolar spaces // Combinatorics'88 / ed. A. Barlotti. Rende: Mediterranean Press, 1988. P. 267-280.

[34] De Clerck F., Van Maldeghem H. On linear representation of $(\alpha, \beta)$-geometries // European J. Combin. 1994. V. 15. P. 3-11.

[35] De Clerck F., Van Maldeghem H. Some classes of rank 2 geometries // Handbook of Incidence Geometry: Buildings and Foundations / ed. F. Buekenhout. Amsterdam: Elsevier, 1995. P. 433-475.

[36] De Clerk F., Thas J. Partial geometries in finite projective spaces // Arch. Math. 1978. V. 30. P. 537-540.

[37] De Clerk F., Thas J. The embedding of $(0, \alpha)$-geometries in $P G(n, q)$. Part I // Ann. Discrete Math. 1983. V. 18. P. 229-240.

[38] Debroey I. Semipartial geometries satisfying the diagonal axiom // J. Geom. 1979. V. 13. P. $171-190$.

[39] Debroey I., Thas J. On polarities of symmetric semipartial geometries // Atti Accad. Naz. Lincei Rend. Cl. Sci. Fis. Mat. Natur. 1977. V. 62. P. 607-612.

[40] Debroey I., Thas J. On semipartial geometries // J. Comb. Theory A. 1978. V. 25. P. $242-250$.

[41] Debroey I., Thas J. Semipartial geometries in $P G(2, q)$ and $P G(3, q) / /$ Atti Accad. Naz. Lincei Rend. Cl. Sci. Fis. Mat. Natur. 1978. V. 64. P. 147-151.

[42] Delandtsheer A. Dimensional linear spaces // Handbook of Incidence Geometry: Buildings and Foundations / ed. F. Buekenhout. Amsterdam: Elsevier, 1995. P. 193-294.

[43] Del Fra A., Ghinelli D. Diameter bounds for locally partial geometries // European J. Combin. 1991. V. 12. P. 293-307.

[44] Del Fra A., Ghinelli D., Pasini A. Diameter bound for an EGQ // J. Combin. Inform. System Sci. 1990. V. 15. P. 256-270.

[45] Del Fra A., Ghinelli D., Pasini A. One diagram for many geometries // Advances in Finite Geometries and Designs. Proc. 3rd Isle of Thorns Conf. Chelwood Gate, UK, 1990 / ed. J. Hirshfeld et al, 1991. P. 111-140.

[46] Del Fra A., Ghinelli D., Hughes D. R. Extended partial geometries with minimal $\mu$ // Geom. Dedicata. 1992. V. 42. № 2. P. 119-128.

[47] Del Fra A., Ghinelli D., Meixner T., Pasini A. Flag-transitive extensions of $C_{n}$ geometries // Geom. Dedicata. 1992. V. 37. №3. P. 253-273.

[48] Deza M., Laurent M. Bouquets of matroids, $d$-injection geometries and diagrams // J. Geom. 1987. V. 29. P. 12-35.

[49] Feit W. Finite projective planes and a question about primes // Proc. Amer. Math. Soc. 1990. V. 108. P. 561-564.

[50] Feit W., Higman G. The nonexistence of certain generalized poligons // J. Algebra. 1962. V. 1. P. 114-131.

[51] Gevaert H. Partiele Meetkunden, Maximale Bogen en Flocks of Kwadratische Kegels // Ph. D. Thesis. Gent: State Univ. Gent, 1987.

[52] Goethals J., Seidel J. The regular two-graph on 276 vertices // Discrete Math. 1975. V. 12. P. $143-158$.

[53] Haemers W. A new partial geometry constructed from the Hoffman-Singleton graph // Finite Geometries and Designs. Proc. 2nd Isle of Thorus Conf., 1980 / ed. P. Cameron et al. Cambridge: Cambridge Univ. Press, 1981. P. 119-127.

[54] Haemers W. Regular two-graphs and extensions of partial geometries // European J. Combin. 1991. V. 12. P. 115-123.

[55] Haemers W. There exist no $(76,21,2,7)$ strongly regular graph // Finite Geometries and Combinatorics / ed. F. De Clerck et al. Cambridge: Cambridge Univ. Press, 1993. P. $175-176$.

[56] Hall J. Classifying copolar spaces and copolar graphs // Quart. J. Math. 1982. V. 33. P. $421-449$.

[57] Hering C. Transitive linear groups and linear groups which contain irreducible subgroups of prime order // Geom. Dedicata. 1974. V. 2. P. 425-460. 
[58] Hill R. Caps and groups // Atti dei convegni Lincei 1976, Colloquio Intern. sulle Teorie Combinatorie, Roma. V. II. P. 384-394.

[59] Higman D. G. Flag transitive collineation groups of finite projective spaces // Illinois J. Math. 1962. V. 6. P. 434-446.

[60] Higman D. G., McLaughlin J. Geometric ABA-groups // Illinois J. Math. 1961. V. 5. P. 382-397.

[61] Hirshfeld J., Thas J. The characterizations of projections of quadrics over finite fields of even order // J. London Math. Soc. 1980. V. 22. P. 226-238.

[62] Hobart S. A., Hughes D. R. Extended partial geometries: nets and dual nets // European J. Combin. 1990. V. 11. P. 357-372.

[63] Hobart S. A., Hughes D. R. EpGs with minimal $\mu$. II // Geom. Dedicata. 1992. V. 42. P. 129-138.

[64] Hoffman A., Singleton R. On Moore graphs of diameter 2 and 3 // IBM J. Res. Develop. 1960. V. 4. P. 497-504.

[65] Huang T. On quasi-semisymmetric designes // Finite Geometry and Combinatorics: Abstracts. Gent: State Univ. Gent, 1997. P. 63-65.

[66] Hughes D.R. Extended partial geometries: dual 2-designes // European J. Combin. 1990. V. 11. P. 459-471.

[67] Huybrechts C., Pasini A. Flag-transitive extensions of dual projective spaces // Bull. Math. Soc. Belg. - Simon Stevin. 1998. V. 5. P. 341-352.

[68] Ivanov A., Shpectorov S. A characterization of the association schemes of Hermitian forms // J. Math. Soc. Japan. 1991. V. 43. P. 25-48.

[69] Kantor W. Symplectic groups, symmetric designs and line ovals // J. Algebra. 1975. V. 33. P. $43-58$.

[70] Kantor W. Strongly regular graphs defined by spreads // Israel J. Math. 1982. V. 41. P. 298-312.

[71] Kantor W. Generalized polygons, SCABs and GABs // Buildings and the Geometry of Diagrams / ed. L.A. Rosati. Berlin: Springer-Verlag, 1986. P. 79-158. (Lecture Notes in Math. V. 181.)

[72] Kantor W. Primitive permutation groups of odd degree, and an application to finite projective planes // J. Algebra. 1987. V. 106. P. 15-45.

[73] Кондратьев А.С., Махнев А. А., Старостин А. И. Конечные группы // Итоги науки и техники. Алгебра. Топология. Геометрия. Т. 24. М.: ВИНИТИ, 1986. С. 3-120.

[74] Lam C., Thiel L., Swiercz S., McKay J. The non-existence of ovals in a projective plane of order 10 // Discrete Math. 1983. V. 45. P. 319-321.

[75] Makhnev A. Pseudogeometric graphs connected with partial geometries pg(4,R,1) // Math. Inst. Oberwolfach. V. 32. Tagungsbericht, 1991. P. 11.

[76] Махнев А.А. О сильно регулярных расширениях обобщенных четырехугольников // Матем. сб. 1993. Т. 184. С. 123-132.

[77] Махнев А. А. Конечные локально GQ(3, 3)-графы // Сиб. матем. журн. 1994. Т. 35. C. $1314-1324$.

[78] Махнев А. А. О псевдогеометрических графах частичных геометрий $p G_{2}(4, t) / /$ Матем. сб. 1995. Т. 187. С. $97-112$.

[79] Makhnev A. Locally GQ(3,5) graphs and geometries with short lines // Intern. Conf. dedicated to the memory of P. Kazimirskii, Abstracts. L'viv, 1995. P. 59-60.

[80] Махнев А. А. О сильно регулярных расширениях обобщенных четырехугольников с короткими прямыми // Дискрет. матем. 1996. Т. 8. С. 31-39.

[81] Махнев А. А. О расширениях частичных геометрий, содержаших малые $\mu$-подграффы // Дискрет. анализ и исслед. операций. 1996. Т. 3. С. 71-83.

[82] Makhnev A. On extensions of partial geometries // Algebra. Proc. of the 3rd International Conference on Algebra. Krasnoyarsk, Russia, 1993 / ed. Yu.L. Ershov et al. Berlin: de Gruyter, 1996. P. 165-169.

[83] Махнев А. А. Расширения GQ(4,2), описание гиперовалов // Дискрет. матем. 1997. T. 9. C. $101-116$. 
[84] Махнев А. А. О псевдогеометрических граффах некоторых частичных геометрий // Вопросы алгебры. Т. 11. Гомель: Изд-во Гомельского ун-та, 1997. С. 60-67.

[85] Махнев А. А. Локально GQ(3, 5)-графы и геометрии с короткими прямыми // Дискрет. матем. 1998. Т. 10. С. $72-86$.

[86] Makhnev A. Partial geometries and the Krein condition // Intern. Math. Congress, Abstracts. Univ. Bielefeld, 1998. P. 282.

[87] Махнев А.А. О псевдогеометрических графах частичных геометрий $p G_{2}(4, t)$, II // Дискрет. матем. (в печати).

[88] Махнев А. А. О группах автоморфизмов частичных геометрий и их расширений // Международный алгебраический семинар, посв. 70-летию кафедры высшей алгебры МГУ. Тез. докл. М.: Изд-во МГУ, 1999. С. 41-42.

[89] Makhnev A.A., Paduchikh D. V. The classification of locally GQ(3,q)-graphs // Intern. Alg. Conf. dedicated to the memory of D.K. Faddeev: Abstracts. St. Petersburg, 1997. P. 84.

[90] Махнев А.А., Падучих Д.В. О структуре локально $\operatorname{GQ}(3,9)$ графов // Дискр. анализ и исслед. операций. 1998. Т. 5. С. 61-77.

[91] Махнев А. А., Падучих Д. В. Вполне регулярные локально GQ $(4,2)$ графы // Межд. алгебр. конф. памяти А.Г. Куроша. Тез. докл. М., 1998. С. 188-189.

[92] Mathon R. The partial geometries pg(5, 7,3) // Congr. Numer. 1981. V. 31. P. 129-139.

[93] Mathon R. A new family of partial geometries // Finite Geometry and Combinatorics: Abstracts. Gent: State Univ. Gent, 1997. P. 83-84.

[94] Meixner T. Construction of chamber systems of type $M$ with transitive automorphism group // Buildings and the Geometry of Diagramms / ed. L.A. Rosati. Berlin: Springer-Verlag, 1986. P. 207-217. (Lecture Notes in Math. V. 181.)

[95] Meixner T. Locally finite Tits chamber systems with transitive group of automorphisms in characteristic 3 // Geom. Dedicata. 1990. V. 35. P. 13-30.

[96] Pasechnik D. V. The triangular extensions of a generalized quadrangle of order $(3,3) / /$ Bull. Math. Soc. Belg. - Simon Stevin. 1995. V. 2. P. 509-518.

[97] Pasechnik D. V. The extensions of the generalized quadrangle of order $(3,9)$ // European J. Combin. 1996. V. 17. P. 751-755.

[98] Pasini A. Diagramms and incidencestructures // J. Combin. Theory. Ser. A. 1982. V. 33. P. 186-194.

[99] Pasini A., Yoshiara S. Flag-transitive Buekenhout geometries // Combinatorics'90 / ed. A. Barlotti et al. Amsterdam: Elsevier, 1992. P. 403-447.

[100] Payne S., Thas J. Finite Generalized Quadrangles. Boston: Pitman, 1984. (Research Notes in Math. V. 110.)

[101] Pellegrino G. Su una interpretazione geometrica dei gruppi $M_{11}$ ed $M_{12}$ di Mathieu e su alcuni $t$ - $(v, k, \lambda)$-disegni deducibili da una $12 \frac{4}{5,3}$ calotta completa // Atti Sem. Math. Fis. Univ. Modena. 1974. V. 23. P. 103-117.

[102] Ronan M. Extending locally truncated buildings and chamber systems // Proc. London Math. Soc. 1986. V. 53. P. 385-406.

[103] Scharlau R. Buildings // Handbook of Incidence Geometry: Buildings and Foundations / ed. F. Buekenhout. Amsterdam: Elsevier, 1995. P. 477-646.

[104] Schulte E. Reguläre Inzidenzkomplexe. II, III // Geom. Dedicata. 1983. V. 14. P. 33-79.

[105] Segre B. Forme e geometrie Hermitiane, con particolare riguardo al caso finito // Ann. Math. Pura Appl. 1965. V. 70. P. 1-202.

[106] Seidel J. Strongly regular graphs with $(-1,1,0)$ adjacency matrix having eigenvalue $3 / /$ Linear Algebra Appl. 1968. V. 1. P. 281-298.

[107] Seidel J. On two-graphs and Shult's characterization of symplectic and orthogonal geometries over GF(2) // TH-Report 73-WSK-02. Technical Univ. Eindhoven, 1973.

[108] Seitz G. Flag-transitive subgroups of Chevalley groups // Ann. of Math. 1973. V. 97. P. 27-56. 
[109] Shult E. Groups, polar spaces and related structures // Combinatorics, Proc. Advanced Study Inst. Breukelen / ed. M. Hall and J. van Lint. V. 55. Amsterdam: Math. Centre Tract, 1974. P. 130-161.

[110] Spence E. Is Taylor's graph geometric? // Discrete Math. 1992. V. 106/107. P. 449-454.

[111] Sprague A. Pasch's axiom and projective spaces // Discrete Math. 1981. V. 33. P. 79-87.

[112] Sprague A. Rank 3 incidence structures admitting dual-linear, linear diagram // J. Combin. Theory. Ser. A. 1985. V. 38. P. 254-259.

[113] Thas J. A. Construction of partial geometries // Simon Stevin. 1972/1973. V. 46. P. 95-98.

[114] Thas J. A. Partial geometries in finite affine spaces // Math. Z. 1978. V. 178. P. 1-13.

[115] Thas J. A. Some results on quadrics and a new class of partial geometries // Simon Stevin. 1981. V. 55. P. 129-139.

[116] Thas J. A. Semi-partial geometries and spreads of classical polar spaces // J. Combin. Theory. Ser. A. 1983. V. 35. P. 58-66.

[117] Thas J. A. Extensions of finite generalized quadrangles // Sympos. Math. 1986. V. 28. P. 127-143.

[118] Thas J., Debroey I., De Clerck F. The embedding of $(0, \alpha)$-geometries in $P G(n, q)$. Part II // Discrete Math. 1983. V. 51. P. 283-292.

[119] Thas J., De Clerck F. Partial geometries satisfying the axiom of Pash // Simon Stevin. 1977. V. 51. P. 123-137.

[120] Tits J. Les groupes de Lie exceptionnels et leur interpretation géométrique // Bull. Soc. Math. Belg. 1956. V. 8. P. 48-81.

[121] Tits J. Sur la trialité et certains groupes qui s'en deduisent // Publ. Math. Inst. Hautes Etudes Sci. 1959. V. 2. P. 14-60.

[122] Tits J. Geometries polyedriques et groupes simples // Atti della II Riunione del Groupement de Mathematiciens d'Expression Latine, Firenze-Bologna (1961), 1963. P. 66-88.

[123] Tits J. Buildings of Spherical Type and Finite BN-pairs. Berlin: Springer-Verlag, 1974. (Lecture Notes in Math. V. 386.)

[124] Tits J. A local approach to buildings // The Geometric Vein. The Coxeter Festschrift. Berlin: Springer-Verlag, 1982. P. 519-547.

[125] Tzanakis N., Wolfskill J. The diophantine equation $x^{2}=4 q^{a / 2}+4 q+1$ with an application to coding theory // J. Number Theory. 1987. V. 26. P. 96-116.

[126] Wallis W. Configurations arising from maximal arcs // J. Combin. Theory. Ser. A. 1973. V. 15. P. $115-119$.

[127] Wilbrink H., Brouwer A. $(57,14,1)$ strongly regular graph does not exist // Indag. Math. 1983. V. 45. P. 117-121.

[128] Wilbrink H., Brouwer A. A characterization of two classes of semipartial geometries by their parameters // Simon Stevin. 1984. V. 58. P. 273-288.

[129] Yoshiara S. On some flag-transitive non-classical c. $C_{2}$-geometries // European J. Combin. 1993. V. 14. P. 59-77.

Институт математики и механики УрО РАН, г. Екатеринбург

Поступила в редакцию

E-mail: makhnev@imm.uran.ru

26.04.1999 